Florida International University FIU Digital Commons

FIU Electronic Theses and Dissertations

University Graduate School

$12-1-2014$

\title{
Engineering Analysis in Imprecise Geometric Models
}

Riccardo Gasparini

rgasp001@fiu.edu

DOI: $10.25148 /$ etd.FI15050211

Follow this and additional works at: https://digitalcommons.fiu.edu/etd

Part of the Applied Mechanics Commons, and the Computer-Aided Engineering and Design Commons

\section{Recommended Citation}

Gasparini, Riccardo, "Engineering Analysis in Imprecise Geometric Models" (2014). FIU Electronic Theses and Dissertations. 1793. https://digitalcommons.fiu.edu/etd/1793

This work is brought to you for free and open access by the University Graduate School at FIU Digital Commons. It has been accepted for inclusion in FIU Electronic Theses and Dissertations by an authorized administrator of FIU Digital Commons. For more information, please contact dcc@fiu.edu. 
FLORIDA INTERNATIONAL UNIVERSITY

Miami, Florida

ENGINEERING ANALYSIS IN IMPRECISE GEOMETRIC MODELS

A dissertation submitted in partial fulfillment of the requirements for the degree of DOCTOR OF PHILOSOPHY

in

MECHANICAL ENGINEERING

by

Riccardo Gasparini 
To: Dean Amir Mirmiran

College of Engineering and Computing

This dissertation, written by Riccardo Gasparini, and entitled Engineering Analysis in Imprecise Geometric Models, having been approved in respect to style and intellectual content, is referred to you for judgment.

We have read this dissertation and recommend that it be approved.

$\begin{array}{r}\hline \text { Cesar Levy } \\ \hline \text { George Dulikravich }\end{array}$

$\begin{array}{r}\hline \text { Giri Narasimhan } \\ \hline \text { Victor Milenkovic } \\ \hline \text { Igor Tsukanov, Major Professor }\end{array}$

Date of Defense: December 1, 2014

The dissertation of Riccardo Gasparini is approved.

Dean Amir Mirmiran
College of Engineering and Computing

Florida International University, 2015 


\section{ACKNOWLEDGMENTS}

I want to thank the Lord for giving to me the strength necessary to continue even in moments of depression ; to my sons Albert, Carlo and Ruben who were there when needed at any time; and especially to the woman of my life, Christina , who morally and e ectively supported my soul at any moment.

I thank also my major professor Dr. Igor Tsukanov and the committee members, Dr. George Dulikravich, Dr.Cesar Levy, Dr.Giri Narasimhan and Dr. Victor Milenkovic for their time and for the critical comments they shared with me. I also thank them for their valuable advice and guidance 


\title{
ABSTRACT OF THE DISSERTATION \\ ENGINEERING ANALYSIS IN IMPRECISE GEOMETRIC MODELS
}

$$
\text { by }
$$

\author{
Riccardo Gasparini
}

Florida International University, 2015

Miami, Florida

Professor Igor Tsukanov, Major Professor

Engineering analysis in geometric models has been the main if not the only credible/reasonable tool used by engineers and scientists to resolve physical boundaries problems. New high speed computers have facilitated the accuracy and validation of the expected results. In practice, an engineering analysis is composed of two parts; the design of the model and the analysis of the geometry with the boundary conditions and constraints imposed on it. Numerical methods are used to resolve a large number of physical boundary problems independent of the model geometry. The time expended due to the computational process are related to the imposed boundary conditions and the well conformed geometry. Any geometric model that contains gaps or open lines is considered an imperfect geometry model and major commercial solver packages are incapable of handling such inputs. Others packages apply di erent kinds of methods to resolve this problems like patching or zippering; but the final resolved geometry may be di erent from the original geometry, and the changes may be unacceptable. The study proposed in this dissertation is based on a new technique to process models with geometrical imperfection without the necessity to repair or change the original geometry. An algorithm is presented that is able to analyze the imperfect geometric model with the imposed boundary conditions using a meshfree method and a distance field approximation to the boundaries. Experiments are proposed to analyze the convergence of the algorithm in imperfect models geometries and will be compared with the same models 
but with perfect geometries. Plotting results will be presented for further analysis and conclusions of the algorithm convergence. 


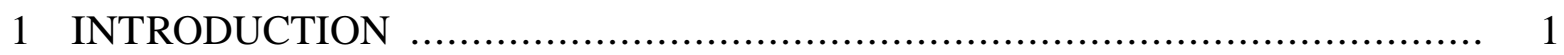

1.1 Engineering analysis in imprecise geometric models........................... 1

1.2 Goals of the Research .............................................................. 5

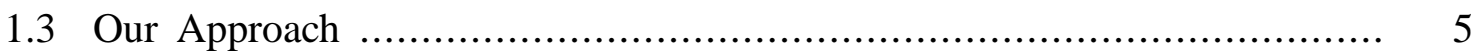

1.4 Contributions (expected results) …........................................... 7

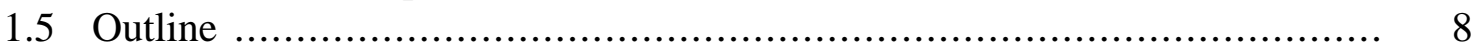

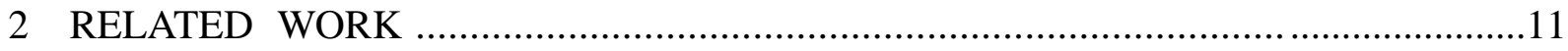

$2.1 \quad$ Geometry healing (repair)............................................................................11

2.2 Solution Structure Method ………………..................................................18

2.2.1 Boundary Value Problem and its Solution Structure .................................18

2.2.2 Treatment of the di erential equation...........................................................22

2.2.3 Examples of Application of SSM to Engineering Problems .........................26

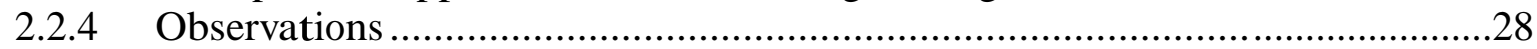

3 PROOF OF CONCEPT: ENGINEERING ANALYSIS IN 2D IMPRECISE

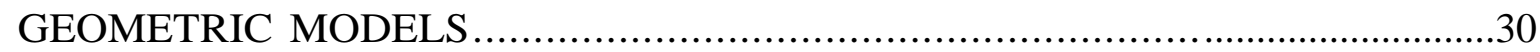

3.0.5 Geometrically adaptive integration..................................................................30

3.0.6 Examples of Application of SSM to Engineering Problems .........................31

3.0.7 Integration over domains with geometric imperfections ...............................34

3.1 Numerical experiments ............................................................................39

3.1.1 Heat conduction in an imperfect geometric model of a heat sink ..................41

3.1.2 2D stress analysis in geometrically imperfect cantilever beam .....................42

3.2 Conclusions and Future Directions................................................................45

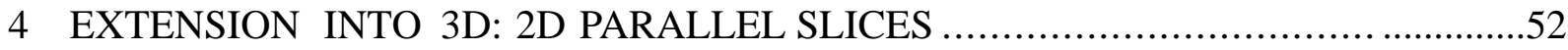

5 VALIDATION AND VERIFICATION OF THE PROPOSED

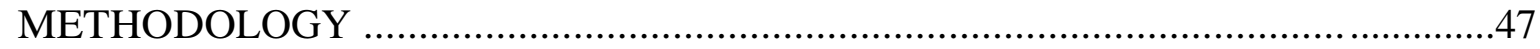

5.1 Volume Computation in Precise and Imprecise CAD Models................. 57

5.1.1 Numerical experiment 1: Volume Computation of a Bracket.........................60

5.1.2 Numerical experiment 2: 3D Simulation in a Cylinder ...............................64

5.1.3 Numerical experiment 3: 3D volume computation in a Spur Gear ................69

5.1.4 Preliminary volume evaluation conclusions.............................................74

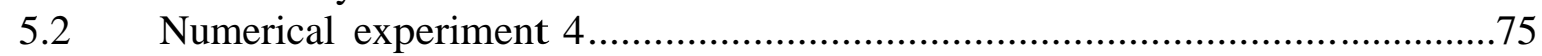

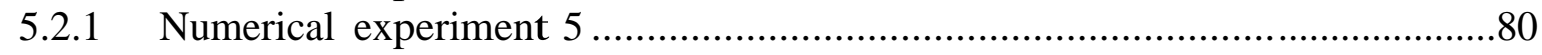

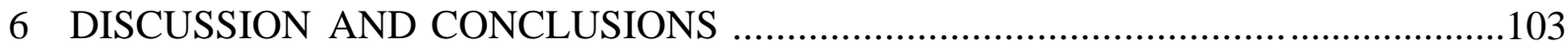

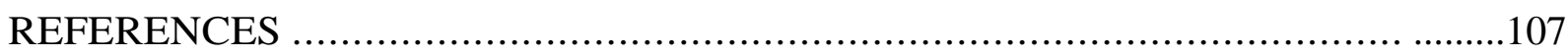

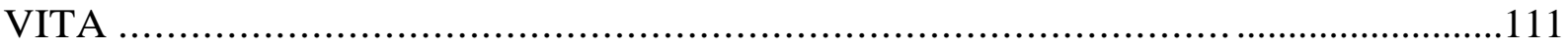




\section{LIST OF FIGURES}

FIGURE

1.1 (a) Inconsistencies and inaccuracies in a geometric representation, such as free edges (edges connected to one face), non-manifold boundaries, and inaccurately computed intersections of surface patches, are accumulated during editing of geometric models. (b) The Noisy Triangulated surface of Michelangelo’s David sculpture contains holes due to the limitations of laser scanning technology.

1.2 (a) Traditional engineering analysis pipeline in imprecise geometric models.

(b) Proposed meshfree engineering analysis pipeline

1.3 (a) A 2D steady-state heat transfer problem with imperfect geometry. (b) PMC values computed for the geometric model in Figure 1.3 (a). Filled circles designate internal points, while non-filled ones correspond to the points outside the geometric model. (c) Integration points are placed using both original geometric model and closing lines.

1.4 (a) Geometrically imprecise model. (b) 3D numerical integration can be performed using 2D integration technique applied to the parallel cross sections of the geometric model. (c) 3D hierarchical space decomposition can be used to perform numerical integration in geometrically imprecise domains.............

2.1 Imprecise Geometry Reconstruction: (a) Mesh Generated by Polygonizing the implicit surface and (b) Filler construction using surface di usion flow... 12

2.2 Zippering Method 14

2.3 Decimation Method 15

2.4 Gap Closing Method

2.5 Minkowski set sum operation.

2.6 Approximate distance fields can be constructed automatically from virtually all geometric representations: (a) triangulated surface of the Michelangelo's David statue and an approximate distance field to it. Blue color corresponds to the boundary points (distance is zero there). (b) CAD model and constructed approximate distance to its surface. Distance field is shown in a cross section. (c) Approximate distance field to the surface of a human femur constructed from segmented Computed Tomography scans.

2.7 Heat transfer in a 2D heat sink: (a) Temperature of $100^{\circ} \mathrm{C}$ is specified at the bottom edge of the geometric domain, and convective heat exchange is given on the rest of the boundaries. Approximate distance fields to (b) Dirichlet and (c) convective boundaries. (d) Temperature distribution predicted by 
the solution structure method.

2.8 Loaded 2D cantilever beam. 26

2.9 Distance fields to the fixed boundaries: (a) in a horizontal direction and (b) in vertical direction. 26

2.10 Computational results provided by the solution structure method: (a) hori- zontal component of the displacement vector; (b) vertical component of the displacement vector; (c) magnitude of the displacement; (d) von Mises stress . 27

3.1 (a) Hierarchical space decomposition is imposed over the geometric domain in Figure 2.8. (b) Basic types of the 2D intersection cases (up to symmetry and rotation transformation). (c) Allocation of the integration points in the integration cells. (d) Positions of the integration points in the geometric domain shown in Figure 2.8.

3.2 A 2D steady-state heat transfer problem with imperfect geometry................

3.3 (a) Heatsink with initial Cartesian grid (blue), one application of geometry cell subdivision and marked regular (gray) and irregular geometry cells (light red). (b) Closure of gaps and non-manifold boundary removal using hierarchical space decomposition: closing lines (dashed lines) are converted into the closing cells (dark red). Non-manifold boundaries are removed by reversing the value of the intersection flag for cell edges.

3.4 Propagation of the PMC values.

3.5 (a) PMC values computed for the geometric model in Figure 3.2. Filled cir- cles designate internal points, while non-filled ones correspond to the points outside the geometric model. (b) Integration points are placed using both original geometric model and closing lines.................................... 36

3.6 Distribution of the temperature field in imprecise geometric model shown in Figure 3.2. .

3.7 Heat transfer in geometrically imperfect model of a heat sink: (a) geometric model and boundary conditions; and (b) resolution of geometric imperfections on a hierarchical space decomposition....

3.8 Temperature distributions in imperfect geometric model shown in Figure 3.7(a) obtained using biquadratic B-splines defined over uniform $35>35$ (coarse) Cartesian grid: (a) without subdividing the geometry cells; (b) with two hierarchical subdivisions of the geometry cells.

3.9 Temperature distributions in imperfect geometric model shown in Figure 3.7(a) obtained using biquadratic B-splines defined over uniform $70>70$ (fine) Cartesian grid: (a) without subdividing the geometry cells; (b) with two hierarchical subdivisions of the geometry cells. 
3.11 Results of structural analysis of a 2D cantilever beam with large geometric defects: (a) magnitude of the displacement vector and (b) distribution of the von Mises stress obtained using a coarse $35>15$ uniform Cartesian grid of bi- quadratic B-splines with no subdivision of the geometry cells. (c) Magnitude of the displacement vector and (d) distribution of the von Mises stress ob- tained using a coarse $35>15$ uniform Cartesian grid of biquadratic B-splines with two hierarchical subdivision of the geometry cells. (e) Magnitude of the displacement vector and (f) distribution of the von Mises stress obtained using a fine $75>25$ uniform Cartesian grid of biquadratic B-splines with two

hierarchical subdivision of the geometry cells.

3.12 Results of structural analysis of a 2D cantilever beam with small geometric defects: (a) magnitude of the displacement vector and (b) distribution of the von Mises stress obtained using a fine $75>25$ uniform Cartesian grid of

biquadratic B-splines with two hierarchical subdivision of the geometry cells..

3.14 Results of computer simulation of the temperature field in geometric model shown in Figure 3.13: (a) coarse initial integration grid results in relatively large cells that contain the valid geometric boundary as well as the closing line. The latter removes the corresponding integration cells and splits the geometric domain into two parts. The integration cells are shown here in gray color. (b) Temperature distribution obtained using the integration cells in Figure 3.14(a). (c) Hierarchical subdivision of the geometry cells makes it possible to separate the geometric boundary and the closing line. In this case the closing line closes the geometric boundary. The integration cells are shown here in gray color. (d) Temperature distribution obtained using the integration cells in Figure 3.14(c).

3.15 (a) Closing line (shown as a red dashed line) intersects the original geometric boundary. (b) Temperature distribution in the geometric domain in Figure 3.15(a). (c) Adding another boundary piece to the geometric model in Figure 3.15(a) changes the way how the closing lines are created. Now they do not intersect with the original geometric boundaries. (d) Temperature distribution in the geometric domain in Figure 3.15(c).

3.16 Resolution of non-manifold boundaries: (a) placement of two irregular cells resolves non-manifold boundaries but creates an "attachment" to the geometric model. (b) By placing two additional irregular cells in the cell which has four edge-boundary intersections non-manifold boundary pieces can be removed. (c) Simulated temperature distribution with removal of "attachments”. 
4.1 (a) Imprecise solid model. (b) 3D numerical integration can be performed using 2D integration technique applied to the Cartesian parallel cross sections of the geometric model. (c) 3D hierarchical space decomposition allow to allocate integration nodes that can be used to perform numerical integration in geometrically imprecise domains

5.1 Precise bracket model. .......................................................... 59

5.2 Imprecise bracket model.................................................. 59

5.3 Precise bracket volume vs grid size......................................... 60

5.4 Integration errors vs grid size. .............................................. 61

5.5 Precise bracket volume vs gauss points. ...................................... 61

5.6 Integration error vs number of gauss points............................... 62

5.7 Imprecise bracket volume vs grid size.................................... 62

5.8 Integration errors vs grid size. .............................................. 63

5.9 Imprecise bracket volume vs gauss points.................................. 63

5.10 Integration error vs number of gauss points................................. 64

5.11 Precise cylinder geometry. ................................................. 65

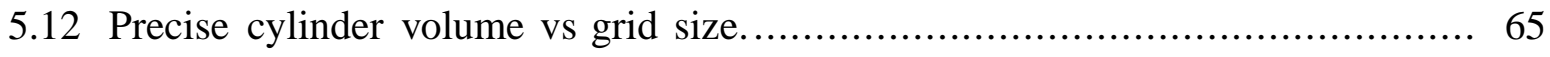

5.13 Integration errors vs grid size. ............................................. 66

5.14 Precise cylinder volume vs gauss points. ..................................... 66

5.15 Integration error vs number of gauss points................................. 67

5.16 Imprecise cylinder geometry.............................................. 67

5.17 Imprecise cylinder volume vs grid size. ................................... 68

5.18 Integration errors vs grid size. ............................................... 68

5.19 Imprecise cylinder volume vs gauss points.................................. 69

5.20 Integration error vs number of gauss points................................ 69

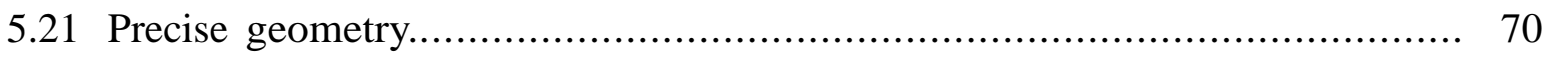

5.22 Spurge volume vs grid size................................................ 71

5.23 Integration errors vs grid size. .......................................... 71 
5.24 Spurge volume vs gauss points.......................................... 71

5.25 Integration error vs number of gauss points................................. 72

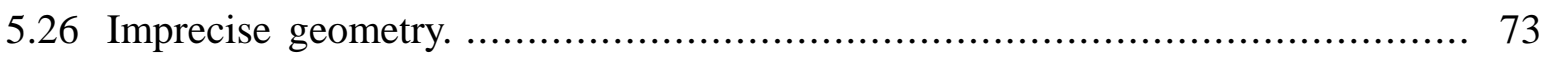

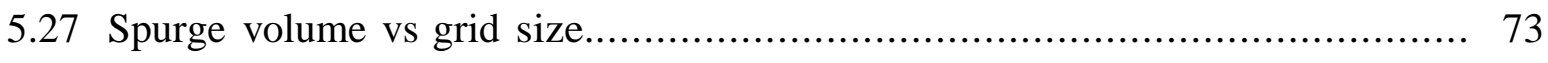

5.28 Integration errors vs grid size. .............................................. 73

5.29 Spurge volume vs gauss points........................................... 74

5.30 Integration error vs number of gauss points................................... 74

5.31 SolidWork Editor for imprecise bracket model. ................................. 76

5.32 Ansys text editor for damage geometric model. ............................ 77

5.33 Imprecise bracket model................................................... 77

5.34 Precise bracket model. ..................................................... 78

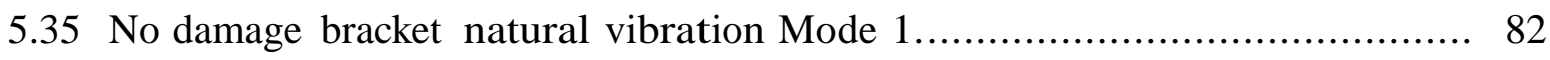

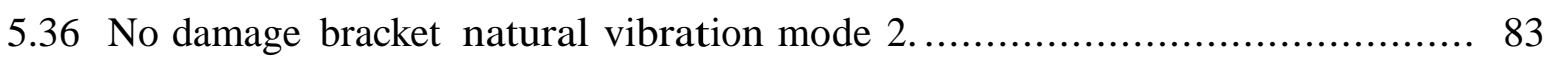

5.37 No Damage Bracket Natural Vibration Mode 3.............................. 83

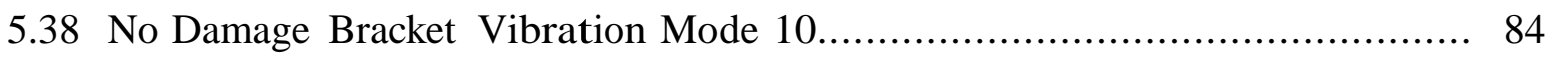

5.39 No Damage Bracket Vibration Convergence Mode 1.............................. 84

5.40 No Damage Bracket Vibration Convergence Mode 2. .......................... 85

5.41 No Damage Bracket Vibration Convergence Mode 3.......................... 86

5.42 No Damage Bracket Vibration Convergence Mode 10.......................... 86

5.43 Damage Bracket SolidWork View........................................ 87

5.44 Damage Bracket Natural Vibration Mode 1.................................. 87

5.45 Damage Bracket Natural Vibration Mode 2. ................................. 88

5.46 Damage Bracket Natural Vibration Mode 3. ................................ 88

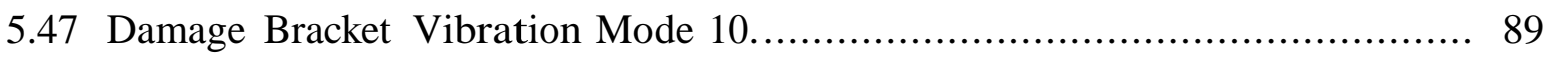

5.48 Damage Bracket Vibration Convergence Mode 1............................... 90

5.49 Damage Bracket Vibration Convergence Mode 2. ............................. 90 
5.50 Damage Bracket Vibration Convergence Mode 3. ................................ 91

5.51 Damage Bracket Vibration Convergence Mode 10............................. 91

5.52 No Damage SpiderBracket Model............................................... 92

5.53 No Damage SpiderBracket Natural Vibration Mode 1.......................... 92

5.54 No Damage SpiderBracket Natural Vibration Mode 2........................... 93

5.55 No Damage SpiderBracket Natural Vibration Mode 3.......................... 94

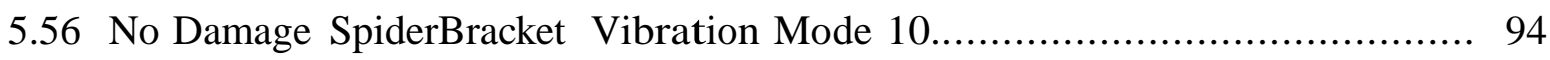

5.57 No Damage Spider Bracket Vibration Convergence Mode 1.................... 95

5.58 No Damage Spider Bracket Vibration Convergence Mode 2.................... 96

5.59 No Damage Spider Bracket Vibration Convergence Mode 3..................... 96

5.60 No Damage Spider Bracket Vibration Convergence Mode 10................... 97

5.61 Damage SpiderBracket Model.............................................. 97

5.62 Damage SpiderBracket Natural Vibration Mode 1.............................. 98

5.63 Damage SpiderBracket Natural Vibration Mode 2. ............................ 98

5.64 Damage SpiderBracket Natural Vibration Mode 3. ............................ 99

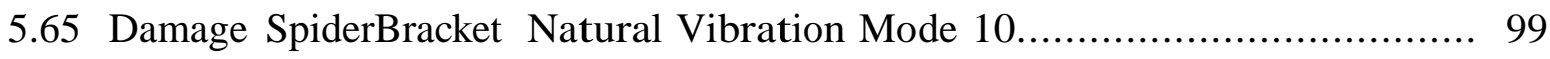

5.66 Damage Spider Bracket Vibration Convergence Mode 1......................... 100

5.67 Damage Spider Bracket Vibration Convergence Mode 2. ......................... 101

5.68 Damage Spider Bracket Vibration Convergence Mode 3.......................... 101

5.69 Damage Spider Bracket Vibration Convergence Mode 10........................ 102 


\section{CHAPTER 1 \\ INTRODUCTION}

\subsection{Engineering analysis in imprecise geometric models}

Engineering analysis is one of the important components of every design process. Engineering analysis accelerates product development, and ensures safety and durability of the products. Currently, the life of almost every product starts from the creation of Computer Aided Design (CAD) geometric model. During design analysis, geometric models are modified to accommodate the changes dictated by the analysis results, and shape optimization,etc. In many cases extensive editing of CAD models and their translation from one CAD system to another result in geometric inaccuracies and inconsistencies [8]. There are two major sources of errors in geometric models: inconsistencies and inaccuracies presented in the geometric representation, and geometric errors due to conversion from one CAD system to another [5].

Inconsistencies and inaccuracies in a geometric representation, such as free edges (edges connected to one face), non-manifold boundaries, sliver faces, and inaccurately computed intersections of the surface patches, are accumulated during editing of geometric models. Some of these geometric imperfections are shown in Figure 1.1(a). Acquired and reverse engineered geometric models, which are represented as a "polygonal soup" [52] may contain holes in their boundaries due to the missing (non-scanned) pieces as shown in Figure (b). (Figure 1.1(b)).

Incorrectly chosen tolerances in the stereolithography (STL) geometric models [1] may produce either gaps or non-manifold boundary. Conversion of geometric models from one CAD system to another may result in loss of semantics information and numerical precision. Because di erent CAD systems use di erent geometric tolerances and geometric algorithms, loss of numerical precision often causes appearance of gaps, misaligned boundaries, and topological inconsistencies in geometric models.. Even though that these geometric imperfections can be very small and almost invisible, they often prevent Finite Element Method (FEM) meshing and direct application of engineering analysis methods based on the finite element paradigm as seen in Figure (a). (Fig- 


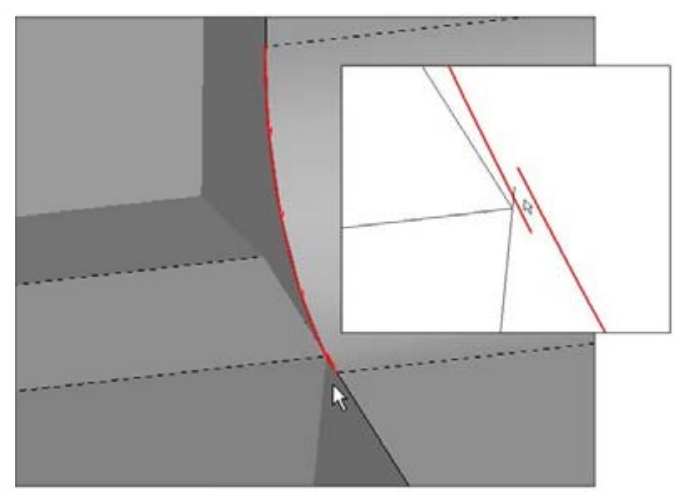

(a)

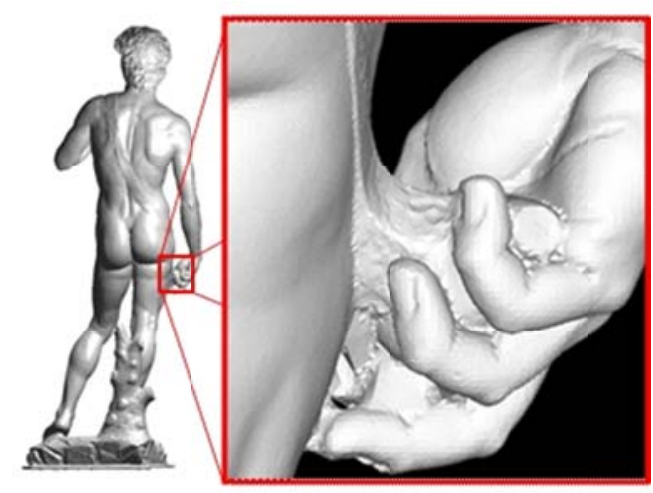

(b)

Figure 1.1: (a) Inconsistencies and inaccuracies in a geometric representation, such as free edges (edges connected to one face), non-manifold boundaries, and inaccurately computed intersections of surface patches, are accumulated during editing of geometric models. (b) The Noisy Triangulated surface of Michelangelo's David sculpture contains holes due to the limitations of laser scanning technology.

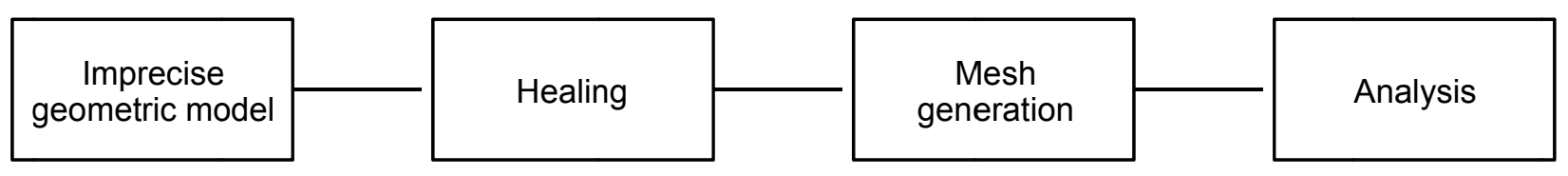

(a)

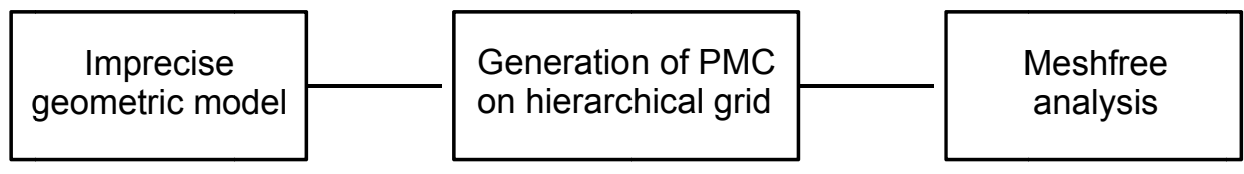

(b)

Figure 1.2: (a) Traditional engineering analysis pipeline in imprecise geometric models. (b) Proposed meshfree engineering analysis pipeline. 
ure 1.2(a)). The need to perform engineering analysis in the presence of geometric inaccuracies and imperfections led to the development of various geometric healing (repair) techniques and tools [16] that can be classified as the volumetric [26], surface [40, 38, 24, 27, 6] and hybrid [3] healing techniques. These tools can represent the surface of the geometric model in the volumetric domain and apply the healing methods to the volumetric model. After fixing geometric imperfections, the volumetric model is transformed back to the surface model using either marching cubes or dual contouring algorithms [21, 17]. Application of volumetric healing methods produces manifold boundaries, but these methods often result in over tesselated geometric boundaries. Surface-based geometric repair techniques perform local modifications of the original model at the places where the geometric errors and inconsistencies have been detected. These techniques are directly applied to the tessellated (usually triangulated) boundary [38]. A surface repair method applied directly on a Boundary representation (B-rep) geometric model was proposed in [24]. Geometric computations are used to determine intersections of the neighboring surface patches, projecting and inserting boundary edges into geometric faces [3]. Gaps between tessellated surface patches are removed by using stitching [27] or zippering [48] algorithms. Surface healing methods often generate small or skewed triangles that lead to poor Finite Element meshes. The fact that automated geometry repair tools [23] have been proposed, in many real-world cases they usually require human supervision and intervention [53]. Geometry repair also requires careful analysis of a variety of special cases of possible geometric defects and the ways they can be removed [56, 4, 6]. Recently, knowledge and learning based geometry healing algorithms were proposed in [39, 20]. An adaptive Cartesian mesh generation approach, based on volumetric geometry healing, was proposed in [53]. The method works well for small geometric imperfections, but it is incapable of removing large geometric defects.In addition it requires user intervention and guidance.

Di culties in creation and handling of spatial meshes suitable for the Finite Element Analysis (FEA) have lead to the development of alternative engineering analysis methods. In contrast to the traditional analysis techniques based on FEM, these new methods, called meshfree or meshless methods, do not require spatial mesh that conforms to 
the shape of the geometric domain Geometric flexibility of the meshfree methods makes them attractive for applications that are di cult for traditional FEA.

In this study we propose and demonstrate an extension of the solution structure method $[32,43,12]$ that enables engineering analysis in imprecise geometric models without reconstructing a valid geometric model. The method uses the original geometric model and no healing is applied as shown in the diagram in Figure 1.2(b). The proposed method requires computations of a Point Membership Classification (PMC) at the nodes of hierarchical space decomposition. These PMC values are then used by a geometrically adaptive integration [22, 43] to place integration or collocation points at which the governing equation is enforced. For the valid geometric models, PMC can be computed by ray casting/stabbing [26] or by computing the sign of a signed distance to the boundaries of a geometric model. However, imprecision in the geometric model prohibit direct computation of PMC. Non-manifold boundary, holes and gaps in the geometric boundary, make the notion of inside and outside undefined. To compute PMC values for imprecise geometric models we adopt an approach similar to the one described in [15]. In contrast to the traditional approaches that produce valid watertight geometry, we will use a composite geometric representation to resolve geometric inaccuracies and compute PMC values without repairing or reconstructing the geometric model. Once PMC values are computed, the original unrepaired geometric model is used to allocate the integration or collocation points.

Besides the governing equation, the solution method has to provide a means to satisfy the prescribed boundary conditions. The salient feature of the structure solution method is the exact treatment of the specified boundary conditions by using solution structures, expressions that combine boundary conditions, basis functions and the functions vanishing on the geometric boundaries. The method is essentially meshfree and does not require construction of spatial meshes that conform to the shape of a geometric model. Also, it does not restrict the choice of the basis functions used for solution approximation or the solution method. References [43, 32, 47, 33] report successful implementation of the solution structure method with classical and trigonometric polynomials, as well as B-splines defined over uniform and non-uniform Cartesian grids. Providing exact 
treatment to the boundary conditions, the method can employ any suitable solution technique to compute the numerical values of the degrees of freedom in the solution. And last, but not least, the solution structure method enables complete automation of the solution procedure [50,43, 12].

\subsection{Goals of the Research}

The main goal of the dissertation work is to develop numerical methods, algorithms and data structures for direct application of engineering analysis tools to imprecise geometric models. In particular, we have focused on the following research objectives:

- Development of e cient geometric and numerical 2D/3D integration algorithms;

- Investigation of how the distance field approach can be used to resolve geometric inconsistencies and irregularities; and

- Application of the developed numerical method to engineering analysis of native CAD models with small geometric errors and features, scanned and reversed engineered shapes, and partially defined conceptual models.

\subsection{Our Approach}

In our recent paper [13] we proposed a novel numerical technique that enables engineering analysis in imprecise geometric models without reconstructing a valid solid model. At the heart of our method lies a modified geometrically adaptive integration technique. The technique uses a hybrid geometric model that consists of hierarchical space decomposition, boundary representation (B-rep), and distance fields. Hierarchical space decomposition helps to resolve the geometric imperfections, while the original geometric model is used to allocate the integration points in the boundary (geometry) cells. Our method uses solution structures that combine together the distance fields to the geometric boundaries, boundary conditions and basis functions to enforce the prescribed boundary conditions. For example, Figures 1.3 (b) and (c) illustrate allocation of the integration points in 2D imperfect geometric domain shown in Figure 1.3 (a) using our new approach. 


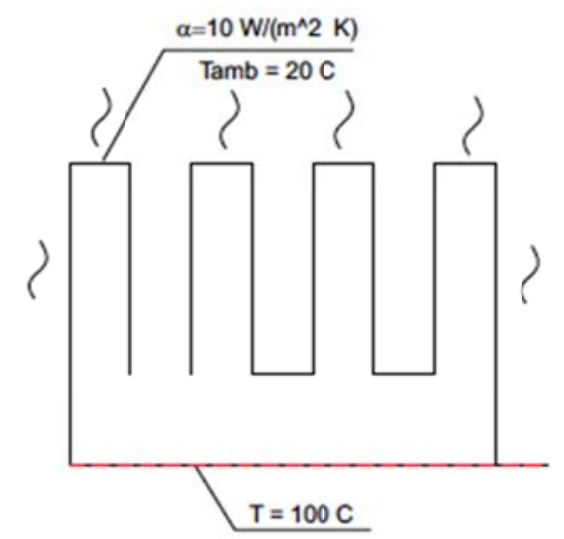

(a)

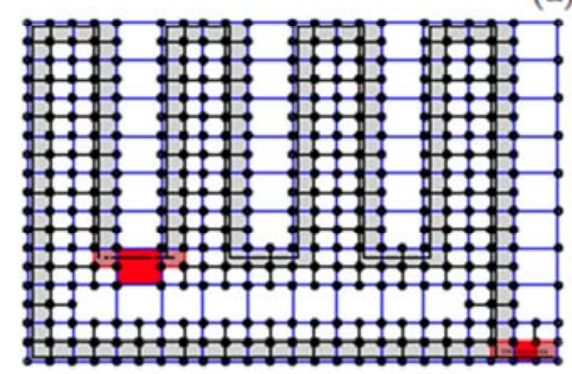

(b)

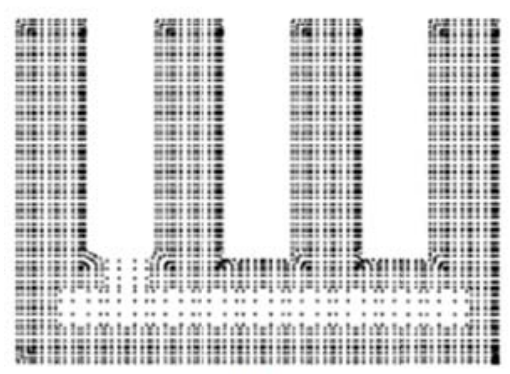

(c)

Figure 1.3: (a) A 2D steady-state heat transfer problem with imperfect geometry. (b) PMC values computed for the geometric model in Figure 1.3 (a). Filled circles designate internal points, while non-filled ones correspond to the points outside the geometric model. (c) Integration points are placed using both original geometric model and closing lines.

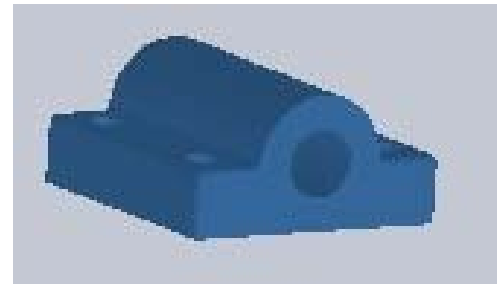

(a)

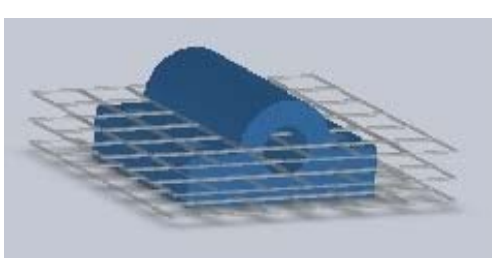

(b)

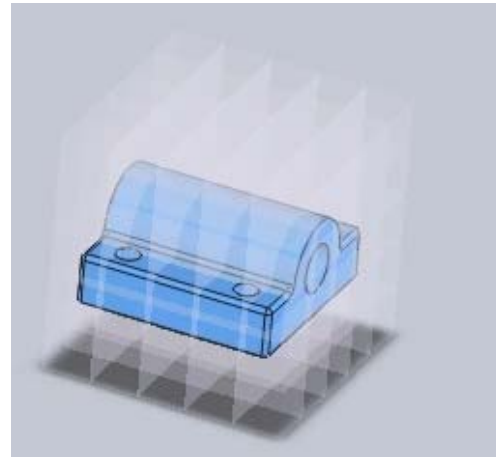

(c)

Figure 1.4: (a) Geometrically imprecise model. (b) 3D numerical integration can be performed using 2D integration technique applied to the parallel cross sections of the geometric model. (c) 3D hierarchical space decomposition can be used to perform numerical integration in geometrically imprecise domains. 
Our approach was also generalized to perform direct engineering analysis in 3D geometric domains with geometric inaccuracies and imperfections. This novel approach is a direct extension of the 2D method. The approach is based on the recognition that any 3D geometric model can be represented by a set of the parallel cross sections (Figure 4.1 (b)) which can be used to perform numerical integration. In any geometry, each cross section will constitute a $2 \mathrm{D}$ geometric domain. In this approach, the geometric inaccuracies are treated and addressed separately in each cross section. To satisfy the prescribed boundary conditions, the approximate distance fields to the 3D boundaries are used. This approach was evaluated against known benchmark solutions for perfect geometric models. We also investigated numerical and computational properties of this technique.

The numerical integration techniques were implemented using $\mathrm{C}++$ programming language and interfaced with the existing software package that implemented the Solution Structure Method (SSM). To demonstrate feasibility of the numerical method a number of benchmark problems with known analytic solutions were solved. We investigated the behavior of the algorithms and computational tools for di erent types of geometric imperfections. In addition, we numerically evaluated the errors caused by the geometric imperfections comparing the solutions in geometrically imperfect models with the solutions obtained in the accurate geometric domains.

In this work we have investigated how the distance field approach is used to resolve geometric inconsistencies and irregularities.

\subsection{Contributions (expected results)}

The main goal of this study is to design and verify a mechanical computational algorithm able to analyze and process models with geometrical imperfections without repairing the model. To obtain this goal it will be first necessary to develop an algorithm in 2D to analyze physical models with geometrical imperfections. Such an algorithm will act as a closing gap tool and will be able to handle the imposed boundaries' conditions not only over perfect domains, but also over geometries with open gaps and extended lines. The algorithm will allow a hierarchical decomposition of the geometry and the allocation of gauss integration points for numerical analysis. A Solution Structure Method 
(SSM) with a distance field approximation (to the boundaries) will be used. After processing a couple of experiments in 2D to determine the e ectiveness of such algorithm, the algorithm will be extended to resolve geometrical models with imperfections in 3D. Also implemented will be a novel integration method using parallel slices over the domain recreating multiple superimposed layers for the allocation points and following the gauss integration method in 3D. The 2D experiments will be evaluated perfect and imperfect geometries in thermal and stress analysis; for 3D experiments we will have a primary approach of this algorithm to evaluate volume value and verify convergence with the real volume value, a major analysis will be addressed to evaluate natural vibration in 3D for perfect and imperfect models. All the computation analysis will be processed without repairing geometries or using fixing techniques common in this field.

\subsection{Outline}

Engineering analysis over geometrical domains in 2D or 3D require a precise representation of the model and its boundaries. The proposed study and experimentation is addressed to models with imperfect geometries either at the surface or at the interior of the geometrical model. To achieve this purpose, a novel algorithm was designed to reach a feasible result without the necessity to repair the model or change the conditions of boundaries. For this goal, di erent procedures will be developed for a final experimentation to verify and assure convergence. In the 2D models a Solution Structure Method (SSM) will be applied using a meshfree formulation with distance fields. For 3D models, a new integration method was employed through slices in 2D using an allocation of gauss points in the 2D parallel grid slices. The algorithm was able to process models with perfect and imperfect geometries without repairing. The generated plots show the convergence of the new slices method with the solutions obtained using a well known commercial package software (SolidWorks).

\section{- Related Work}

The first part of the study, linked to related works done previously by other researchers, focuses on repairs to a damaged geometric model without taking into 
consideration the possibility of distortion of the boundaries conditions applied. These repairs were proposed to correct geometrical models used for artistic scope usually in cinematography when recovering images. The same technique was applied for analysis of engineering models without preventing the obvious change of the boundaries condition, a high price to pay due to mathematical analysis consequences.

- Proof of Concept in 2D

After describing the di erent attempts to reconstruct damage to the geometrical model, we present a method based on a mathematical solution structure (SSM) which lies in a modified geometrical adaptive integration technique using a hybrid geometric model that is conformed by hierarchical space decomposition, boundary representation, that will be called B-rep, and distance fields which are the implicit functions that hold the geometrical distance information to the boundaries. This first mathematical approach is applied to 2D geometrical domains. Studies for thermal and stress problems are presented and show the feasibility of the method to be applied in 3D cases.

\section{- Extension into 3D}

The same algorithm applied to a $2 \mathrm{D}$ boundary value problem was extended to the 3D case. The novel algorithm slices the geometric 3D model in di erent and selected numbers of $2 \mathrm{D}$ planes. We will use a hierarchical space decomposition of the geometric model and will compute a Point Membership Classification (PMC) to address geometric errors and allocate integration points in 3D cells using geometrically adaptive integration algorithms. The method to treat gaps and geometrical imperfections is described in order to allocate integration points. No geometrical domains are changed and enforcement of the boundaries conditions are respected.

\section{- Validation and Verification}

The 2D problem is presented in two cases with a thermal and stress mechanical analysis of both of them with geometrical imperfections. The 3D problems was 
computed and validated as precise and imprecise volume models using a bracket, cylinder and a spur gear. A major mechanical analysis in the field of natural vibration problems in 3D was proposed for precise and imprecise models using a bracket and a spider bracket. Experiments showed the e ectiveness of the proposed algorithm to analyze geometric damage models without geometric repairs and the impossibility to process the same damaged geometries using well known commercial solver packages.

- Conclusion

We analyze our method for both 2D or 3D imprecise geometrical models. Results were compared between precise and imprecise models using graphical charts able to analyze and endure the convergence results. The SolidWorks mechanical analysis package was used to obtain values for perfect models and was compared with our 3D method for imperfect geometrical models. 


\section{CHAPTER 2}

\section{RELATED WORK}

Methods based on computational geometry require precise and large quantities of data to generate accurate polygonal models. Vertices of these polygonal models are the acquired data points. Methods for data acquisition techniques from computer vision like stereo range images and space carving can generate 3D dimensional points sets but are imprecise when compared with laser or optical scanners [37]. Surface reconstruction using algorithms such as (GPDE) Geometric Partial Di erential Equations to solve surface modeling problems use a mean curvature flow, averaged mean curvature flow and surface di usion flow. All of these equations are nonlinear and they do not depend upon any particular parameterizations. Algorithms dealing with non-stable borders have been used [58]applying a sweep-line to determine the inconsistent borders and its eventual correction. Geometric modeling with implicit surfaces remains an active research area. Developments in this field include level set methods, variational implicit surfaces, and adaptively sampled distance field. Methods in implicit surface modeling, using an approximate interpolation set of data points via Radial Basis Function (RBF) and Moving Least Square (MLS)[39] are especially useful for repairing lack of data information in implicit surfaces since no topological constraints are required.

\subsection{Geometry healing (repair)}

Three of the most used method for geometric healing are: reconstructing surfaces by volumetric regularization [7]; discrete surface modeling using geometric flows using PDF (Partial Di erential Equations)[55]; and reconstruction 3D scattered data interpolation with compactly supported basis functions [57]. Reconstruction surfaces by volumetric regularization is commonly used in computer vision applications. Computer vision technique has been used largely to acquire three-dimensional data from images. The techniques involved include: shape from shading, depth approximation from a pair of stereo images, and volumetric reconstruction from images at multiple viewpoints and areas easily convertible to a polygonal description. The use of cameras makes the process easier since they are inexpensive resources when compared to laser and optical scanners. The a ordable cameras for vision-based techniques enable the creation of digital models 


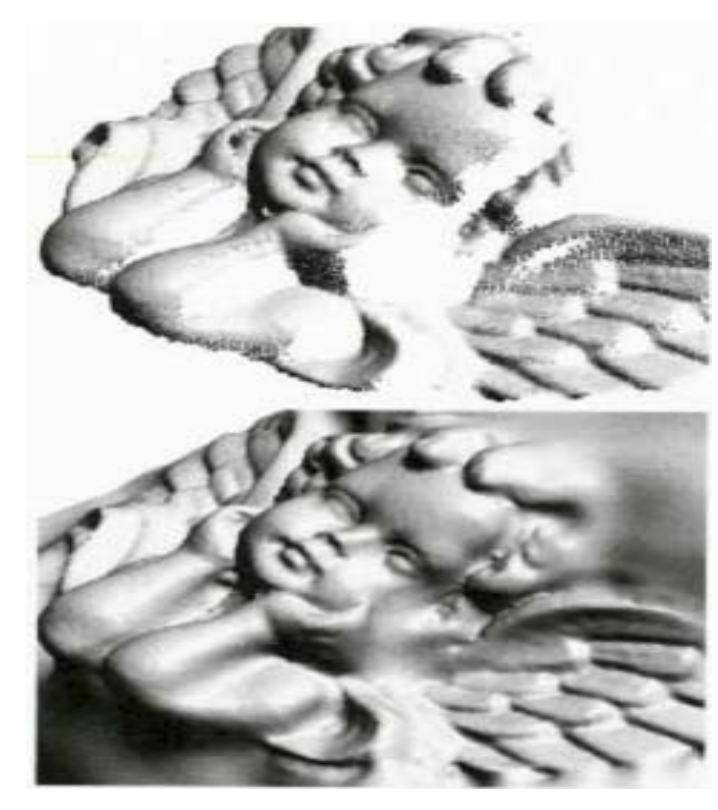

(a)

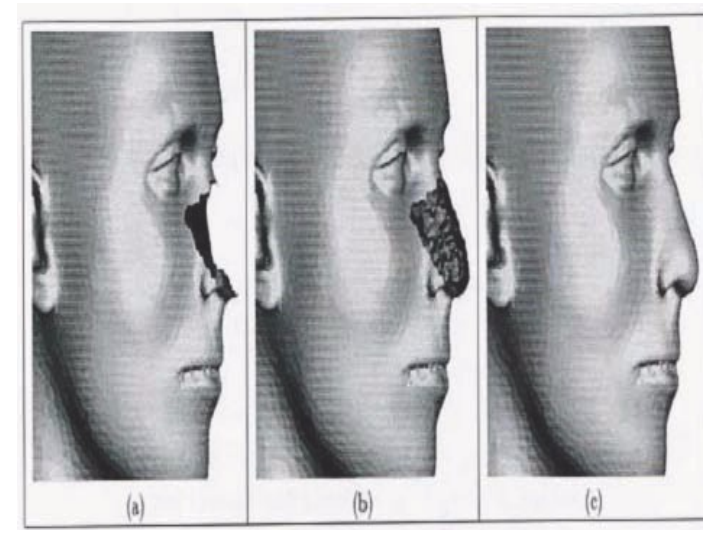

(b)

Figure 2.1: Imprecise Geometry Reconstruction: (a) Mesh Generated by Polygonizing the implicit surface and (b) Filler construction using surface di usion flow.

by computer users without any professional CAD skills required. Noise from data acquisition is large compared with laser scan technique. Typical reconstruction methods are: alpha shapes[14]; crust [2]; and region growing algorithms.In the case of volumes carved from multiple viewpoints, the reconstructed models remain in the volumetric domain having artifacts due to the voxel discretization. Volumetric regularization is obtained using the advantages over existing algorithms. Implicit functions constructed estimate the surface well in regions where there is little data. The reconstructed surface is insensitive to noise in data acquisition because we can allow the surface to approximate, better than exactly interpolate the data points, and the reconstructed surface is locally detailed, yet globally smooth, because of the use of Radial Basis Functions that achieve multiple orders of smoothness.

Discrete surface modeling using partial di erential equations of various curvature-driven Geometric Partial Di erential Equations (GPDE) is used to solve several surface modeling problems. The GPDS method is used to include mean curvature flow, averaged mean curvature flow, and surface di usion flow. Equations are nonlinear and geometry 
intrinsic. The technique involved in reconstructing a surface interpolates the points or curves or the boundaries of patches with a specified order of continuity. The surfacefitting problem is the most general and includes the surface blending and $\mathrm{N}$-sided filling problems.

Reconstruction of 3D scattered data interpolation with compactly supported basis functions uses a hierarchical approach to 3D scattered data interpolation with compactly supported basis functions. Interpolation and approximation of scattered data with RBF has a variational nature which supplies a user with a rich palette of radial basis functions. The basic question is whether to choose local or global RBF. Fitting scattered data by local compactly-supported RBF leads to a simpler and faster computation procedure while a practical usage of global RBF is based on sophisticated mathematical techniques such as the fast multipole method [57].Global RBF are extremely useful in repairing incomplete data while approaches based on compactly supported RBF are sensitive to the density of interpolated/approximated scattered data. Careful selection of RBF influence domains controlled by certain parameters is required.

Di erent methods have been proposed for healing surface meshes coming from geometric reconstruction using any well known data acquisition technique as mentioned in the section for healing geometric models. Zippered Polygon [48] is applied when digitizing the shape in 3D. The method consist in:

- Align the meshes with each other using modified iterated closest-point algorithm,

- Zipper together adjacent meshes to form a continuous surface that correctly captures the topology of the object, and

- Compute local weighted averages of surface positions on all meshes to form a consensus surface geometry.

This method[48] was proposed to be applied in:

- Digitizing complex objects for animation and visual simulation.

- Digitizing the shape of a found object such as an archaeological artifact for measurement and for dissemination to the scientific community. 

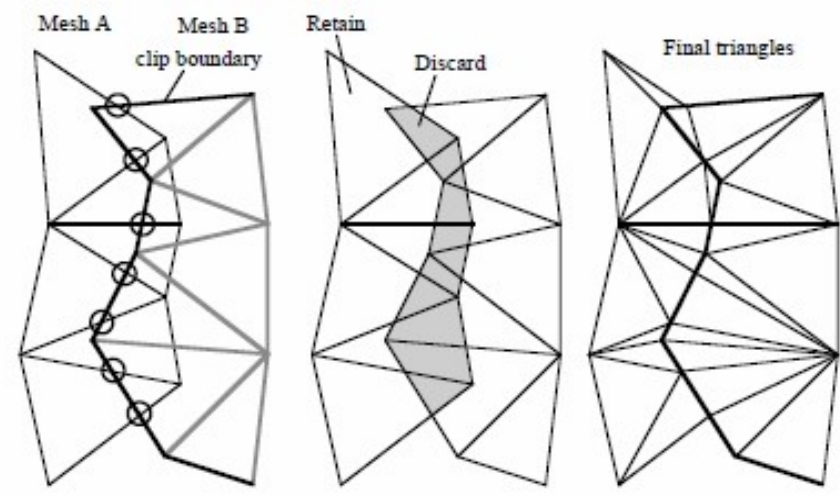

Figure 2.2: Zippering Method

- Digitizing human external anatomy for surgical planning.

- Digitizing the shape of a damaged machine part to help create a replacement.

An old method for digitizing consisted of painstakingly touching a 3D sensing probe to thousands of positions of the object and manually specifying the connectivity of these points. Actually, range scanners[48] make feasible and lest tedious this operation.Range scanners are devices that sense 3D positions on an object's surface and return an array of distance values. A range image is an mxn grid of distances (range points) that describe a surface either in Cartesian coordinates or cylindrical coordinates, with two of the coordinates being implicitly defined by the indices of the grid. Di erent techniques can be used to create a range image. Structured light, time of flight lasers, radar, sonar, and shading are samples of these techniques. However, the complicated shape of an object can obscure the process of the range images and can force the whole operation to process more than one range image to many of them. The zippered polygon meshes method uses a mesh of triangles to represent the range image data at all stages. Each sample point in the mxn range image is a potential vertex or node in the triangle mesh. Two processes are used to create the joint portion area: registration and integration. . Registration [48] refers to computing a rigid transformation that brings the points of one range image into alignment with the portions of a surface that is share with another range images. Integration [48]is the process of creating a single surface representation from the sample points of two or more range images. Before joining a pair of meshes the 
portions that are redundant in the new meshed joint area are removed. This method is in continued improvement due to the new algorithms application and the new range image approximation, able to o er better visual results.

Mesh decimation methods [54]were used for 3D acquisition and modeling detailed geometric models. The objectives of mesh decimation algorithms are to reduce the number of triangles in the whole triangle mesh in order to keep the original topology, and to better approximate the original geometry. Using searching pass algorithms each vertex of the triangle is stored as a possible candidate to be eliminated. If it meets the adopted decimation criteria, the vertex and all the triangles connected to the vertex are canceled. The resulting empty space left by the decimation is patched using a local triangulation. The vertex decimation criteria searching continue until a termination condition is reached. The termination criteria are generally specified as a percent reduction of the original mesh and a fixed maximum decimation value.

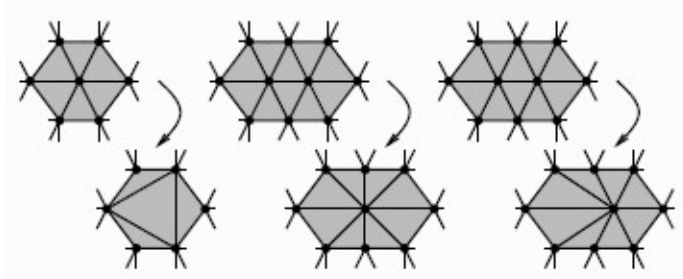

Figure 2.3: Decimation Method

Progressive gap closing method [28] was proposed to eliminate issues that occur during the mesh boundary decimation task. Applying the well-known decimation methods to the complexity meshes generated during acquisition data and modeling results in severe artifacts due to the lack of consistent connectivity information, T-vertices, narrow gaps and cracks. The progressive gap closing method proposes to add a vertex pair contraction to join unconnected regions of the mesh. The method introduces a vertex-edge operation providing a support for closing gaps and stitching together the boundaries of triangle patches located near each other. The decimation process is error controlled and guided progressively in terms of error. The objective idea behind this algorithm [28]is that the gap closing procedure is executed by utilizing the vertex-edge contraction operator using 
the mesh-decimation methodology to boundaries targeted at gap closing.

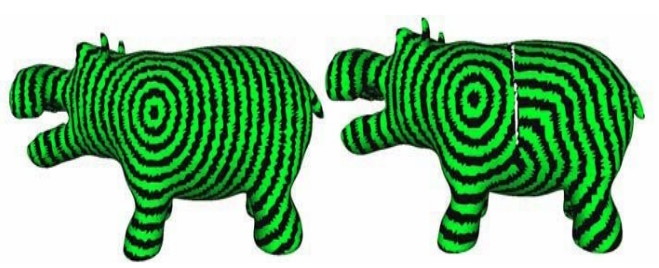

Figure 2.4: Gap Closing Method

It was proposed as a method to repair arbitrary polygon models which guarantees to produce a closed surface [41] that partitions the space into disjoint internal and external volumes. The method works under the premise to have a model represented as a polygon soup and construct an inside/outside volume using an octree grid and reconstruct the surface by contouring [41] which produces a polygonal approximation of the zero-values isosurface. Contouring algorithms can be classified in two types. The primal methods proposed by [21] extract polygons by connecting points lying on the grid lines creating blobby surfaces and curved corners; and the hybrid methods that allow polygon vertices to be placed inside the grid cells to reproduce sharp edges and corners.

Visualization technology has been developed over the last 30 years in a very rushed track. Computational mechanical analysis has been largely improved and accepted due to the well-known interactions between the mechanical analysis and the way to visualize the object under physical stresses and its responses. However both of them, visualization and mathematical analysis, have a di erent practical and theoretical meaning. Computational visualization technology was developed essentially to repair new or existing data acquisition from surfaces under reconstruction due to damage or lack of visual precision. Data obtained from scanners or cameras contain noise due to mechanic or electromagnetic perturbation during the information acquisition show a bad visual quality. Industries like gaming, filmmaking, and advertising are samples of businesses that require perfect visualization. Computational mechanical analysis require a real model even if imperfect. Unfortunately, the methods to repair and visualize the models that have been used, and are still in operation, change the original shape of the model damaging any mathematical data obtained during analysis. Visualization of surfaces using 
mesh simplification like decimation or re-tiling eliminates vertex, edges and faces to simplify the model adding a new mesh using a local re-triangulation. The patching method eliminates intrinsic areas like corners or smooth angles that are essential and common elements in mechanical engineering analysis. The vertex clustering method based in geometric proximity regroups vertices into clusters and computes new vertexes conforming a new external shape and changing the real location and value of the boundaries' conditions. For the purpose of this work to analyze mechanical parts under an imperfect geometric model, a hierarchical octree (3D) will be used with a grid automatically generated and purged at various levels and then converted into boundary representation. Another method to repair imperfect geometries, especially in 3D models, is the Minkowski approximation. It is based on a sum operation of two planar objects defined as the set of all vectors' sums generated by all pairs of curved points which have the same curve normal direction and can be applied as a 3D sum of polyhedral objects which consist in the decomposition of the polyhedral objects into convex pieces. This generates pair-wise convex sums and computes their union. Minkowski sum of two sets Q1 and Q2 is the set of points q1 +q2 - q1 () Q1, q2 () Q2.By definition if C1(t) = (x1(t),y1(t)) and $\mathrm{C} 2(\mathrm{~s})=(\mathrm{x} 2(\mathrm{~s}), \mathrm{y} 2(\mathrm{~s}))$ be two planar regular parametric curves. The convolution curve $\mathrm{C} 1{ }^{*} \mathrm{C} 2$ is defined by: $\left(\mathrm{C} 1{ }^{*} \mathrm{C} 2\right)(\mathrm{t})=\left(\mathrm{C} 1(\mathrm{t})+\mathrm{C} 2(\mathrm{~s}(\mathrm{t}))\right.$. where $\mathrm{C} 1^{\prime}(\mathrm{t})$ is parallel to $\mathrm{C} 2^{\prime}(\mathrm{s}(\mathrm{t}))$, and $[\mathrm{C} 1$ '(t),C2'(s(t))] are greater than zero. Minkowski sum has many applications in the field of robot motions as a collision-free computation path that is called convolution, computer aided design and manufacturing. For the purpose of this work, convolution can be applied to geometrical imperfect models in order to repair the boundaries of the models since following the definition of Minkowski sum and given two planar curved objects as $\mathrm{O} 1$ and $\mathrm{O} 2$, their sum are defined as the set of all vector sums generated by all pairs of points in $\mathrm{O} 1$ and O2. In Figure 2.5,the blue area is the Minkowski set sum of the green and brown smaller areas. The Minkowsky sum of two planar objects considers all points in the interiors as well as on the boundaries of the two objects. Following the scope of this work, the Minkowsky approach could be used as a method to repair damaged models since the sum of convex curves could overlap damaged boundaries, either exterior boundaries or interior boundaries. However, the application of this algorithm 
could modify the original geometrical model [51]; cases like geometric repairs of the contour for artistic purpose can be wide acceptable under the visual point of view; for the scope of this study Minkowski sum could incur in material addition to the geometrical model, which is what we are trying to avoid.

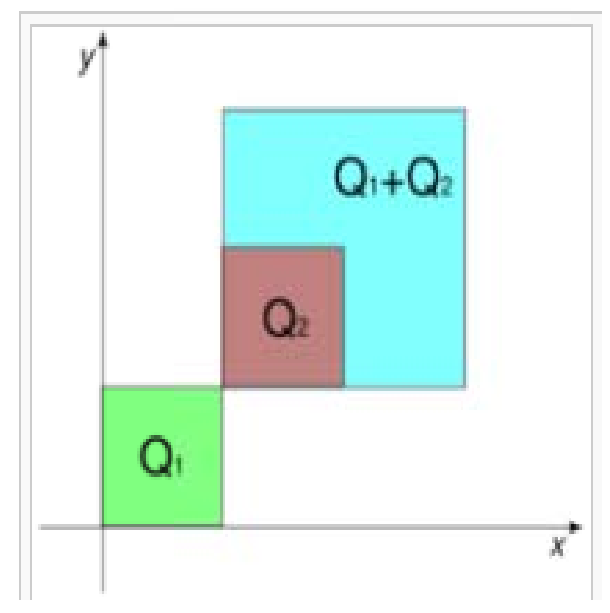

Figure 2.5: Minkowski set sum operation.

\subsection{Solution Structure Method}

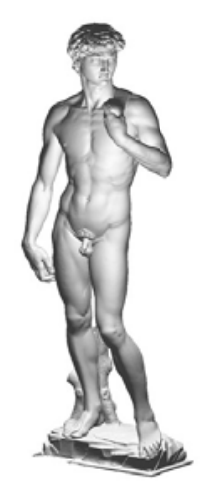

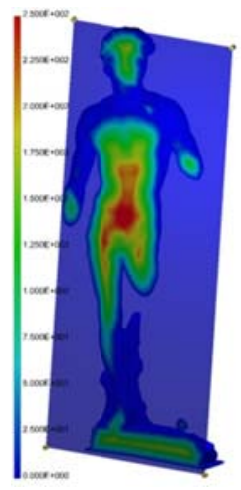

(a)

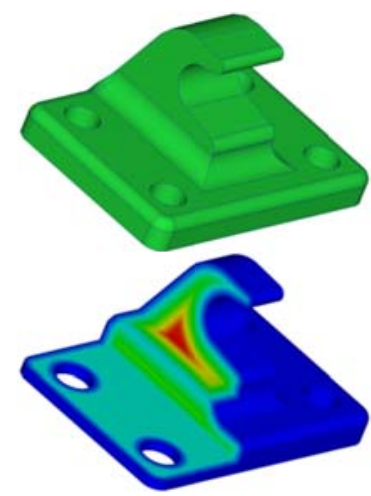

(b)

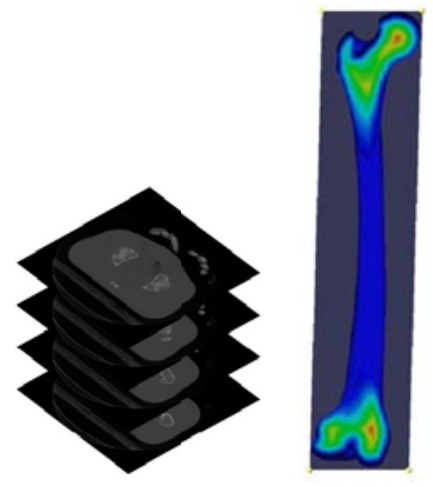

(c)

Figure 2.6: Approximate distance fields can be constructed automatically from virtually all geometric representations: (a) triangulated surface of the Michelangelo's David statue and an approximate distance field to it. Blue color corresponds to the boundary points (distance is zero there). (b) CAD model and constructed approximate distance to its surface. Distance field is shown in a cross section. (c) Approximate distance field to the surface of a human femur constructed from segmented Computed Tomography scans. 


\subsubsection{Boundary Value Problem and its Solution Structure}

The Solution Structure Method was originated by Kantorovich in 1950s. He proposed a simple technique to satisfy Dirichlet boundary condition

$$
\left.u\right|_{\partial \Omega}=\phi
$$

using functions that vanish on the geometric boundaries. According to Kantorovich, a function that satisfies condition (5.3) can be written in the following form [49]:

$$
u=\omega \Phi+\phi,
$$

where $\omega$ is a function that takes on zero value on the boundary $\partial \Omega$ and has non-zero gradient there; function $\phi$ extends Dirichlet boundary condition (5.3) inside the geometric domain $\Omega$. Since $\left.\omega\right|_{\partial \Omega}=0$, the boundary condition (5.3) is satisfied at all the points of the geometric boundary exactly, regardless of the chosen function $\Phi$. The main purpose of $\Phi$ is to satisfy the governing equation of the problem. In order to achieve this $\Phi$ is represented by a linear combination of the basis functions:

$$
\Phi={ }_{i=1}^{n} C_{i} \chi_{i} .
$$

Being substituted into (2.2), $\Phi$ results in a set of functions which satisfy the boundary condition (5.3) exactly regardless of the numerical values of the degrees of freedom $\left\{C_{i}\right\}_{i=1}^{n}$. To find an approximate solution to the boundary value problem, any suitable numerical method can be used. Usually application of a numerical method result in an algebraic system whose solution assigns numerical values to the degrees of freedom.

Kantorovich method had limited applicability mostly because it was not clear how to construct function $\omega$ that vanishes on the boundaries of complex geometric objects. With invention of $R$-functions by Rvachev [30, 31] in 1963 Kantorovich method was reborn. Using $R$-functions it was possible to construct such functions for complex geometric domains represented either by Constructive Solid Geometry or Boundary Representation. 
Another step forward in the development of the Solution Structure Method was understanding that approximate distance functions(fields)that can be used as $\omega$ functions. Approximate distance fields can be constructed either by direct interpolation or least square fit to the samples of Euclidean distance to the geometric boundary [12, 11]. For example, plots in Figure 2.6 show isolines of the approximate distance fields that describe the boundary of Michelangelo's David statue (Figure 2.6(a)), CAD model of an anchor plate (Figures 2.6(b)) and human femur (Figures 2.6(c)).

Also, Rvachev and his students generalized Kantorovich's method for other types of boundary conditions that include Neumann, Robin, Stefan-Boltzmann as well as interface boundary conditions [31, 44, 47, 32, 19,46]. Rvachev proposed a systematic way to construct solution structures - approximate solutions to the boundary value problems that satisfy the prescribed boundary conditions exactly. The main idea of Rvachev's approach was to represent solutions to the boundary value problems by powers of the functions that vanish on the geometric boundaries [32]:

$$
u(\omega)=u(0)+\sum_{k=1}^{m} \frac{1}{k !} u_{k}(0) \omega^{k}+\omega^{k+1} \Phi .
$$

This representation is a straightforward generalization of a classical Taylor series, where the term $\left|x-x_{o}\right|$, measures the distance to the point $x_{o}$, is replaced by $\omega$ which measures the distance to a set of points. Similarly, the $k$ th order derivatives of the function $u$ in the classical Taylor series are replaced by coe cients $u_{k}$ that are $k$ th order derivatives of the function $u$ in the direction $n$ normal to the boundary of a geometric domain. In contrast with a classical Taylor series, where the coe cients are constants, $u_{k}(x, y, z)$ in the expression (2.4) may be arbitrary functions. This also holds when $\omega$ represents ap-proximate distance to the geometric boundary. Taylor series (2.4) provides a connection between the value of a physical field at any spatial point and values of the field and its normal derivatives prescribed on the boundary of a geometric domain. In the context of engineering analysis this means that the function $u$ given by expression (2.4) satisfies specified boundary conditions exactly. 
Generalized Taylor series (2.4) are used to derive solution structures for di erent types of boundary conditions. For example, solution structure for the Neumann boundary condition

$$
\left.\frac{\partial u}{\partial n}\right|_{\partial \Omega}=\varphi
$$

can be written as follows:

$$
u=\Phi_{1}-{ }_{1} \omega D^{\omega}\left(\Phi_{1}\right)+\phi \omega+\omega^{2} \Phi_{2}
$$

where $\left.D_{1}^{\omega}()=\nabla \omega \cdot \nabla\right)$ is the first order di erential operator in the direction normal to the boundary. The first two terms in expression (2.6) represent the unspecified value of the solution $u$ on the domain's boundary. Subtracting $\omega D^{\omega}\left(\Phi_{1}\right)$ from $\Phi_{1}$ assures that the first normal derivative of the di erence vanishes on $\partial \Omega$ [32]. Product of the prescribed function $\phi$ and approximate distance field $\omega$ takes care of the boundary condition (2.5), while the remainder $\omega^{2} \Phi_{2}$ guarantees completeness of the solution structure (2.6). Un- determined functions $\Phi_{1}$ and $\Phi_{2}$ are usually represented by a linear combination of the basis functions (2.3). In the case when basis functions with a compact support, such as, for example, tensor product B-splines, are used, the last term in the solution structure, $\omega^{2} \Phi_{2}$ can be omitted. Similarly, the solution structure that satisfies Robin boundary condition

$$
\left.\left(\frac{\partial u}{\partial n}+h u\right)\right|_{\partial \Omega}=\psi
$$

can be written as follows:

$$
u=\Phi-{ }_{1} \omega D^{\omega}(\Phi)+\psi \omega-h \omega \Phi .
$$

In the context of heat transfer problems, functions $h$ and $\psi$ in the boundary condition (2.7) incorporate the ambient temperature of the environment $T_{a m b}(p)$, thermal conductivity $\lambda(p)$, and convective coe cient $\alpha(p): h=\alpha(p) / \lambda(p)$ and $\psi(p)=$ 
$\alpha(p) T_{a m b}(P) / \lambda(p)$, which are the functions of spatial coordinates.

When functions $\phi, h$ and $\psi$ in the boundary conditions $(5.3,2.5,2.7)$ are specified in a piecewise manner, i.e. di erent functions are prescribed over di erent pieces of the boundary, these functions can be combined into a single function that transfinitely interpolates the given functions [19]. Transfinite interpolation combines approximate distance fields $\omega_{i}$ to the portions $\partial \Omega_{i}$ of the geometric boundary $\partial \Omega$ with the prescribed functions $\phi_{i}[19]$ :

$$
\varphi=\sum_{i=1}^{n} \varphi_{i} \frac{\prod_{j=1 ; j \neq i}^{n} \omega_{j}^{\mu_{j}}}{\sum_{k=1}^{n} \prod_{j=1 ; j \neq k}^{n} \omega_{j}^{\mu_{j}}},
$$

where exponents $\mu_{j}$ are used for interpolating the functions together with their derivatives. More information on transfinite interpolation and its properties can be found in [19]. Transfinite interpolation can be applied to the solution structures as well. For example, the solution structure for mixed boundary conditions

$$
\left.u\right|_{\partial \Omega_{1}}=\varphi ;\left.\quad\left(\frac{\partial u}{\partial n}+h u\right)\right|_{\partial \Omega_{2}}=\psi
$$

is obtained via transfinite interpolation of the individual solution structures:

$$
u=\frac{1}{\omega_{1}+\omega_{2}^{2}}\left(\left(\omega_{1} \Phi_{1}+\varphi\right) \omega_{2}^{2}+\left(\Phi_{2}-\omega_{2} D_{1}^{\omega_{2}}\left(\Phi_{2}\right)+\psi \omega_{2}-h \omega_{2} \Phi_{2}\right) \omega_{1}\right),
$$

where $\omega_{1}$ and $\omega_{2}$ are approximate distance fields to the boundaries $\partial \Omega_{1}$ and $\partial \Omega_{2}$ respectively. Sometimes another solution structure that satisfies mixed boundary conditions (2.10) is used:

$$
u=\omega_{1} \Phi+\varphi-\omega D_{1}^{\omega_{2}}\left(\omega_{1} \Phi\right)-\omega D_{1}^{\omega_{2}}(\varphi)-h \omega_{1} \omega \Phi-h \varphi \omega+\psi \omega,
$$

where $\omega$ is a distance field to the whole geometric boundary, and $\omega_{1}$ is a distance field to the Dirichlet boundary.

\subsubsection{Treatment of the di erential equation}

As we have discussed earlier, using solution structures to represent the physical 
fields being modeled enables exact treatment of the prescribed boundary conditions. 


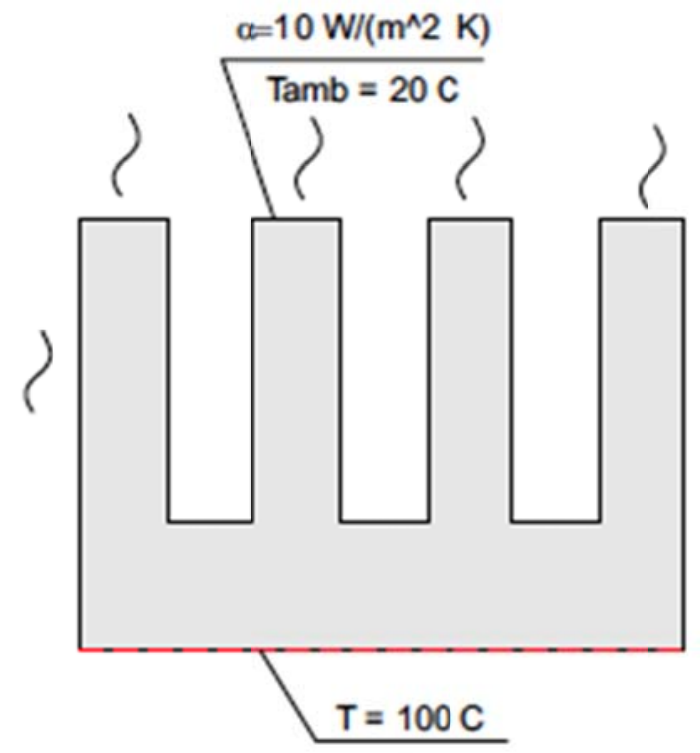

(a)

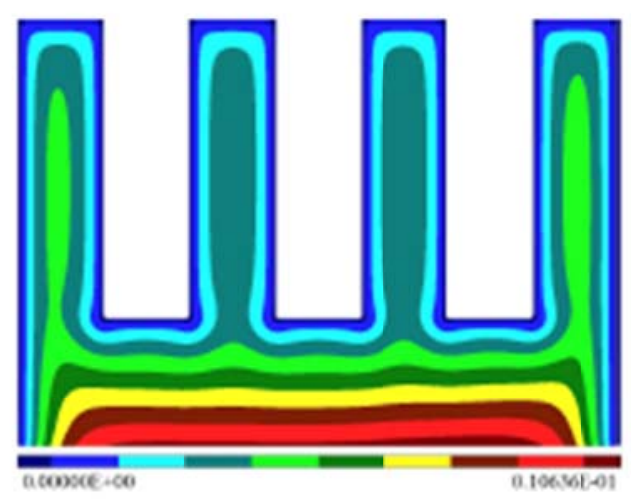

(c)

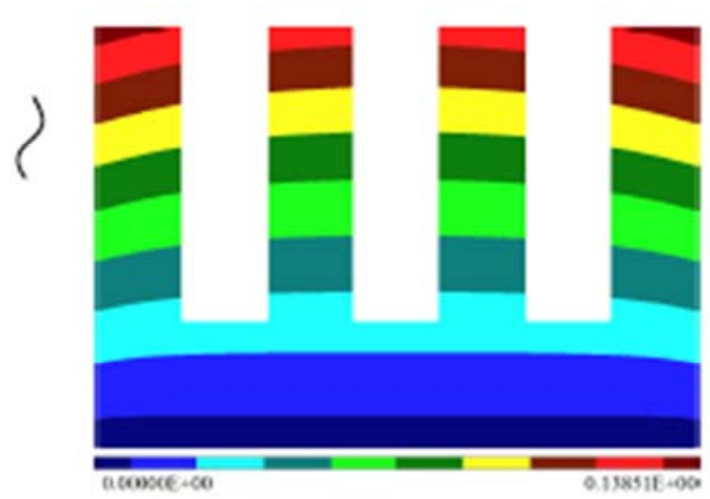

(b)

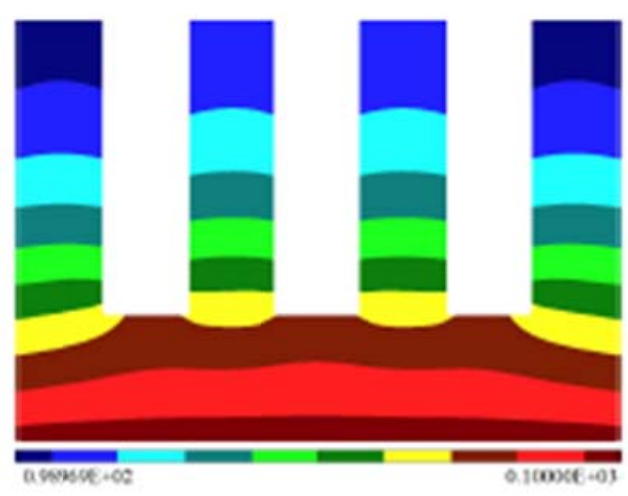

(d)

Figure 2.7: Heat transfer in a 2D heat sink: (a) Temperature of $100^{\circ} \mathrm{C}$ is specified at the bottom edge of the geometric domain, and convective heat exchange is given on the rest of the boundaries. Approximate distance fields to (b) Dirichlet and (c) convective boundaries. (d) Temperature distribution predicted by the solution structure method. 
The governing equation of a problem can be satisfied by a proper choice of the undetermined functions $\Phi, \Phi_{1}$ and $\Phi_{2}$ in the solution structures. If these functions can be determined precisely we have the exact solution of the problem. Unfortunately, it can be done only in a few special cases. In practical situations, however, the undetermined functions are approximated by a linear combination of the basis functions $\left\{\chi_{i}\right\}_{i=1}^{N}$ (2.3).In this case, each solution structure defines a set of functions that satisfy the prescribed boundary conditions. Application of an appropriate solution method makes it possible to determine numerical values of the degrees of freedom in (2.3)such that the solution structure provides the best approximation to the governing equation of the problem. Solution structure method does not restrict the choice of the basis functions or the solution method used to compute numerical value of the degrees of freedom. It has been successfully implemented using polynomials (classical and trigonometric), tensor product B-splines, as well as finite element basis functions in conjunction with Galerkin, Least Squares and collocation methods [34, 36, 44, 47, 12, 46, 42]. For example, Galerkin method is often used for solving heat conduction problems. Applied to Laplace equation $\nabla u=0$ with mixed boundary conditions (2.10) combination of the solution structure and Galerkin methods produces a system of linear algebraic equations $\mathbf{A C}=\mathbf{B}$ in which the elements of the conductivity matrix and the load vector are computed using the following expressions:

$$
\begin{aligned}
a_{i j} & =\iint_{\Omega} \nabla \xi_{i} \nabla \xi_{j} d \Omega+\int_{\partial \Omega_{2}} h \xi_{i} \xi_{j} d S ; \\
b_{i} & =-\iint_{\Omega} \nabla \xi_{i} \nabla u_{1} d \Omega+\int_{\partial \Omega_{2}} \xi_{i}\left(\psi-h u_{1}\right) d S,
\end{aligned}
$$

where $u_{1}=\phi-\omega D^{\omega_{2}}(\phi)-h \phi \omega+\psi \omega$ is a portion of the solution structure which satisfies the non-homogeneous boundary conditions. The solution structure operator transforms

the chosen basis functions $\left\{\chi_{i}\right\}_{i=1}^{N}$ into a new system of basis functions $\left\{\xi_{i}\right\}_{i=1}^{N}$ that satisfy homogeneous boundary conditions of the same type:

$$
\xi_{i}=\omega_{1} \chi_{i}-{ }_{1} \omega D^{\omega_{2}}\left(\omega_{1} \chi_{i}\right)-h \omega_{1} \omega \chi_{i}
$$


Application of the solution structure method to structural analysis problems is often formulated as balance between the work performed by the external forces and the potential energy of the elastic deformation:

$$
\int_{\Omega} \boldsymbol{\epsilon}^{T} \boldsymbol{\sigma} d \Omega=\int_{\Omega} \mathbf{u}^{T} \mathbf{F} d \Omega+\int_{\Gamma_{t}} \mathbf{u}^{T} \mathbf{T} d \Gamma_{t},
$$

where $\boldsymbol{u}=\left(u_{x}, u_{y}, u_{z}\right)^{T}$ is a displacement vector, strain $\boldsymbol{\epsilon}$ and stress $\boldsymbol{\sigma}$ are second order tensor quantities represented by six-dimensional vectors according to the Voigt notation. Boundary conditions are formulated as the prescribed displacements specified over boundary $\Gamma_{u}$ and the traction load specified on the boundary $\Gamma_{t}$. In addition to the boundary forces the geometric model can be subject to the body (gravity) force $\boldsymbol{F}$.

To satisfy the kinematic (essential) boundary condition displacement $\boldsymbol{u}$ can be represented by the following solution structure

$$
\boldsymbol{u}=\sum_{i=1}^{n} C_{i}\left(\omega^{x}, \omega^{y}, \omega^{z}\right)^{T} \chi_{i}+\boldsymbol{u}^{*},
$$

where $\omega^{x}, \omega^{y}, \omega^{z}$ are functions that vanish on the boundaries on which essential boundary conditions are prescribed: and vector valued function $u^{*}=\left(u_{r}^{*}, u_{y}^{*}, u_{z}^{*}\right)^{T}$ interpolates all non-zero displacements in $x, y$ and $z$ coordinate directions respectively. Designating $\left(\omega^{x}, \omega^{y}, \omega^{z}\right)^{T} \chi_{i}$ as the new basis functions $\boldsymbol{\eta}_{i}$ that satisfy homogeneous Dirichlet (essential) boundary conditions, the system of the linear algebraic equations, from which numerical values of the degrees of freedom $\boldsymbol{C}_{i}$ are determined, can be written as follows [12]:

$$
\begin{aligned}
& -\sum_{i=1}^{n} \mathrm{C}_{i} \underbrace{\int_{\Omega} \mathrm{B}^{T}\left[\boldsymbol{\eta}_{i}\right] \mathrm{DB}\left[\boldsymbol{\eta}_{j}\right] d \Omega}_{\text {stiffness coefficient }}=\underbrace{-\int_{\Omega} \boldsymbol{\eta}_{j} \mathbf{F} d \Omega}_{\text {load due to body force }}+ \\
& \underbrace{\int_{\Omega} \mathbf{B}^{T}\left[\boldsymbol{\eta}_{j}\right] \mathrm{DB}\left[\boldsymbol{u}^{*}\right] d \Omega}_{\text {load due to applied displacements }}-\underbrace{\int_{\Gamma_{t}} \boldsymbol{\eta}_{j} \mathbf{T} d \Gamma_{t}}_{\text {load due to applied loads }},
\end{aligned}
$$


where $\boldsymbol{B}$ denotes the matrix of derivatives, also known as the strain-displacement matrix, so that $\boldsymbol{\epsilon}=\boldsymbol{B}[\boldsymbol{u}]$, and $\boldsymbol{D}$ represents the stress-strain matrix so that $\boldsymbol{\sigma}=\boldsymbol{D} \boldsymbol{\epsilon}$. Weak formulations of boundary value problems $(2.13,2.16)$ require numerical integration over the given geometric domain which is performed by using the integration lattice rules that place integration points inside the geometric model.

Besides weak formulations, the solution structure method can be used in conjunction with the collocation method [42]. In this case, solution structure is used to satisfy the prescribed boundary conditions while the governing equation is enforced at the collocation points allocated inside the geometric domain. Our numerical experiments suggest that using solution structures to enforce the prescribed boundary conditions improves convergence of the approximate solutions and may result in a better conditioned algebraic system [45].

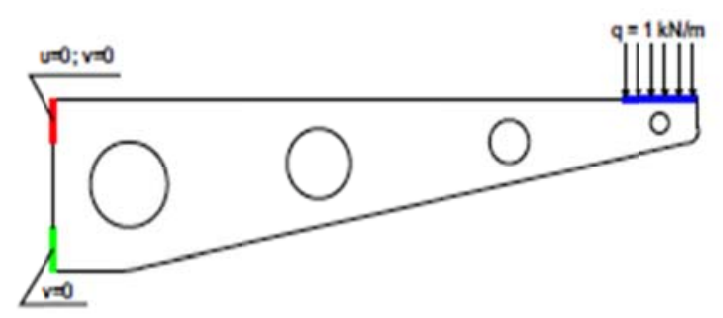

Figure 2.8: Loaded 2D cantilever beam.

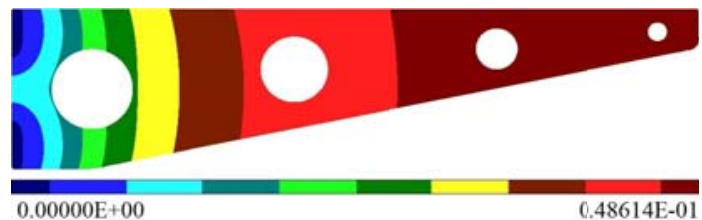

(a)

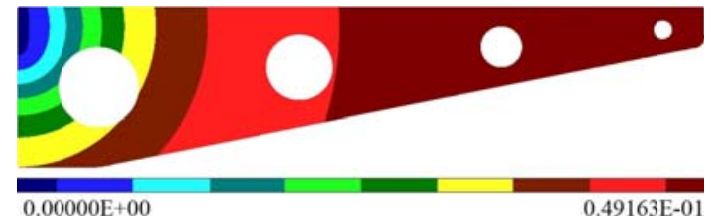

(b)

Figure 2.9: Distance fields to the fixed boundaries: (a) in a horizontal direction and (b) in vertical direction.

\subsubsection{Examples of Application of SSM to Engineering Problems}

Let us demonstrate an application of the Solution Structure Method to heat conduction and plane stress analysis problems.

For heat transfer problems, let us consider heat conduction in a 2D heat sink which is shown in Figure 2.8(a). A temperature of $100^{\circ} \mathrm{C}$ is specified on the boundary 


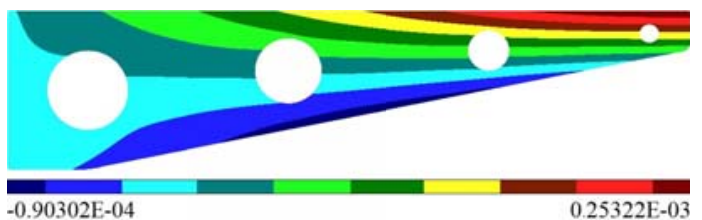

(a)

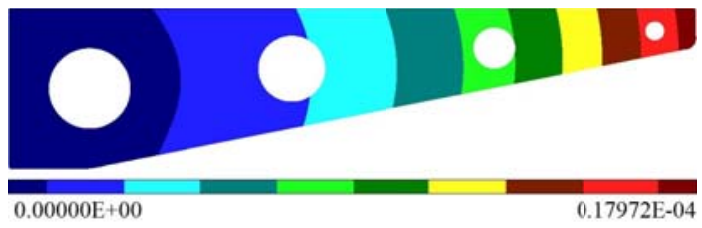

(c)

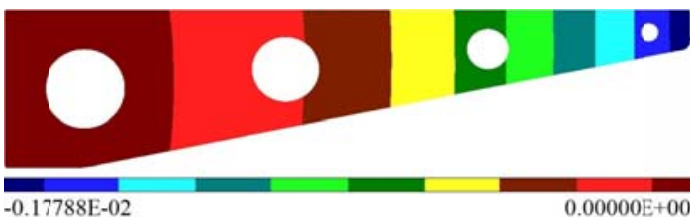

(b)

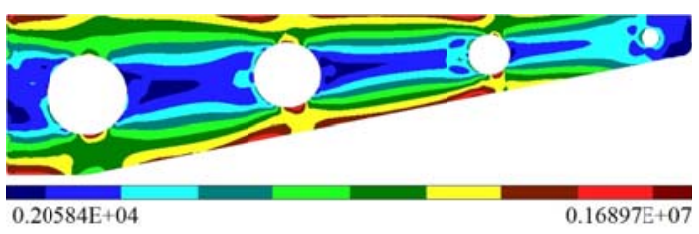

(d)

Figure 2.10: Computational results provided by the solution structure method: (a) horizontal component of the displacement vector; (b) vertical component of the displacement vector; (c) magnitude of the displacement; (d) von Mises stress

$\partial \Omega_{1}$. On the rest of boundaries, convective heat exchange is given:

$$
\left.T\right|_{\partial \Omega_{1}}=100 ;\left.\quad\left(\frac{\partial T}{\partial n}+\frac{\alpha}{\lambda}\right)\right|_{\partial \Omega_{2}}=\frac{\alpha T_{a m b}}{\lambda}
$$

The specified values of the convection coe cient, $\alpha$ and ambient temperature, $T_{a m b}$ are $10 \frac{W}{m^{2} K}$ and $20^{\circ} \mathrm{C}$ respectively. The heat conductivity, $\lambda$, of the material (Aluminum) is $200 \frac{W}{m K}$. The SSM solution procedure starts with analyzing the type of boundary conditions and construction of the solution structure that enforces them. In our case we will represent temperature, $T$, using a solution structure (2.11) that satisfies the mixed boundary conditions (3.1). The solution structure (2.11) contains approximate distance fields $\omega_{1}$ and $\omega_{2}$ that vanish on the boundaries where Dirichlet and Robin boundary conditions are prescribed. These functions are constructed using the original geometric representation. In our case, $\omega_{1}$ and $\omega_{2}$ are constructed from the Boundary Representation of the geometric domain in Figure 2.7(a). Plots in Figures 2.7(b) and (c) present isolines of $\omega_{1}$ and $\omega_{2}$ respectively. Blue color in these plots illustrates the position of the zero set of the approximate distance fields. Once approximate distance fields and solution structure are constructed, application of Galerkin method results in an algebraic system $A C=B$ in which elements of the matrix $A$ and vector $B$ are computed using expression (2.13).The numerical solution of the algebraic problem delivers numerical values of the 
degrees of freedom in the solution structure. Finally, the solution, $T$, can be computed and visualized. Figure 2.7(d) illustrates distribution of the computed temperature.

The next example illustrates application of the solution structure method to plane stress analysis problems. Geometry of a cantilever beam, applied load and fixations are shown in Figure 2.8. Geometric boundary shown in red is fixed in both coordinate directions while the boundary green is fixed in the horizontal direction only. To satisfy the kinematic boundary conditions the components of the displacement vector are represented by the following solution structures:

$$
u_{1}=\omega_{1} \sum_{i=1}^{N} C_{i}^{1} \chi_{i} ; \quad u_{2}=\omega_{2} \sum_{i=1}^{N} C_{i}^{2} \chi_{i} .
$$


As in the previous examples, approximate distance fields $\omega_{1}$ and $\omega_{2}$ are constructed from Boundary Representation of the geometric domain shown in Figure 2.8. Their isolines are shown in Figures 2.9(a) and (b). The plots in blue correspond to the boundaries whose motion is restricted in horizontal and vertical directions. The basis functions $\chi_{i}$ are chosen to be tensor product B-splines defined over 60 $\times 30$ uniform Cartesian grid.

Application of the solution method results in numerical values of the degrees of freedom

$C_{i}^{1}$ and $C_{i}^{2}$. Substituting them back into solution structures (3.2) we can evaluate and

visualize the components of the displacement vector as well as the distribution of the von Mises stress (see plots in Figure 2.10).

\subsubsection{Observations}

As we have seen,the solution method, which enforces the governing equation of the problem, requires sampling integration or collocation points inside the geometric model.It can be done relatively easily if the geometric model constitutes a valid solid model.In the presence of the geometric imperfections (holes, dangling boundaries, self- intersecting boundaries etc.), notions of "inside" and "outside" are not defined.As a result, this precludes application of the solution method that enforces the governing equation.In the next section,we will describe a geometrically adaptive integration tech- nique that can be used to sample integration/collocation points inside geometric domains with geometric imperfections without performing computationally expensive geometry healing. 


\section{CHAPTER 3}

\section{PROOF OF CONCEPT: ENGINEERING ANALYSIS IN 2D IMPRECISE GEOMETRIC MODELS}

\subsubsection{Geometrically adaptive integration}

In $[43,22]$ we described a geometrically adaptive volumetric integration technique which can be used in conjunction with the solution structure method to assemble the algebraic system. The integration process starts with an allocation of a Cartesian grid over the geometric model. The grid is constructed in a such way that the its cells are aligned with the supports of the basis functions (Figure 3.1(a)). Once cells are allocated, they are classified with respect to their position relative to the geometric model. This classification splits the cells into three groups: external, internal, and geometry cells. It can be performed by analyzing the positions of the cells vertices with respect to the geometric model. For example, all vertices of the external cells have to be located outside of the geometric model. Similarly, all vertices of an internal cell must be situated inside the geometric model. Geometry cells have some of their vertices inside the geometric model and some outside. A grid cell can also be classified as a geometry cell if it encloses the boundaries of a geometric domain while having all its vertices inside or outside the geometric domain.

Once the classification phase is over, the integration points are allocated in internal and geometry cells, and numerical integration is performed. During the integration phase external cells are simply ignored. Since internal cells are rectangles in 2D and cuboids $3 \mathrm{D}$, the integration points in these cells are placed in accordance with lattice integration rules [29]. Allocation of the integration points in geometry cells, due to their arbitrary geometric shape, requires special attention. As we demonstrated in [22], a proper space parameterization in geometry cells can substantially reduce the integration error The choice of the space parametrization depends on how the geometric boundary intersects the integration cell $[43,22]$. . In our implementation of the geometrically adaptive integration we use a cell classification similar to the one employed by the marching cubes 
technique [21]. Basic types of the 2D intersection cases (up to symmetry and rotation transformation) are presented in Figure 3.1(b). Depending on the type of the intersection case di erent space parameterizations are employed. For example, a polar coordinate system is used if the integration cell has one or three vertices inside the geometric domain, as shown in Figure 3.1(c). In the other cases, Cartesian coordinates are used to allocate the integration points. To integrate in 3D space spherical, cylindrical and Cartesian coordinate systems are employed. Some intersection cases are ambiguous (like the right-most case in Figure 3.1(b)) - The positions of internal and external vertices allow multiple intersection configurations. In such cases, the ambiguity is resolved by applying hierarchical subdivisions to the cell. The cells are also hierarchically subdivided if they contain small geometric features [22]. Figure 3.1(d) illustrates allocation of the integration points inside geometric domain shown in Figure 2.8.

This geometrically adaptive integration assumes that the geometric model provides the ability to compute a Point Membership Classification (PMC) - a function that returns a relative position of a given point in the space with respect to the geometric model. Most geometric engines support PMC computations for valid solid models. In the presence of geometric imperfections, direct computation of the PMC becomes impossible and the geometric model requires healing.

\subsubsection{Examples of Application of SSM to Engineering Problems}

Let us demonstrate an application of the Solution Structure Method to heat conduction and plane stress analysis problems. Consider heat conduction in a 2D heat sink which is shown in Figure 2.8(a). A temperature of $100^{\circ} \mathrm{C}$ is specified on the boundary $\partial \Omega_{1}$. On the rest of boundaries convective heat exchange is given:

$$
\left.T\right|_{\partial \Omega_{1}}=100 ;\left.\quad\left(\frac{\partial T}{\partial n}+\frac{\alpha}{\lambda}\right)\right|_{\partial \Omega_{2}}=\frac{\alpha T_{a m b}}{\lambda}
$$




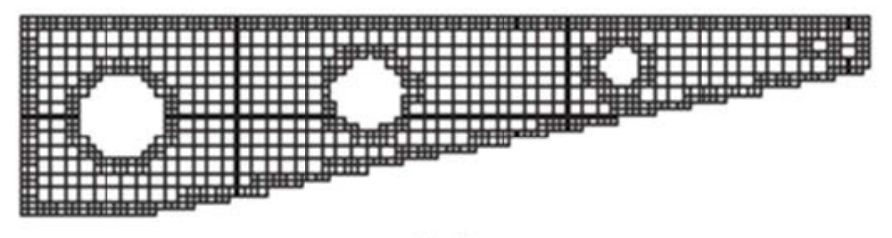

(a)

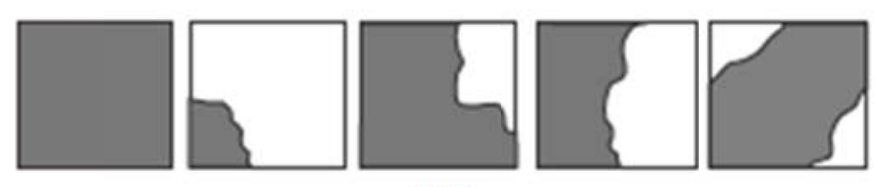

(b)

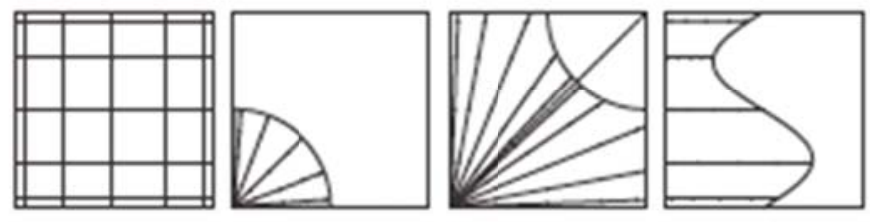

(c)

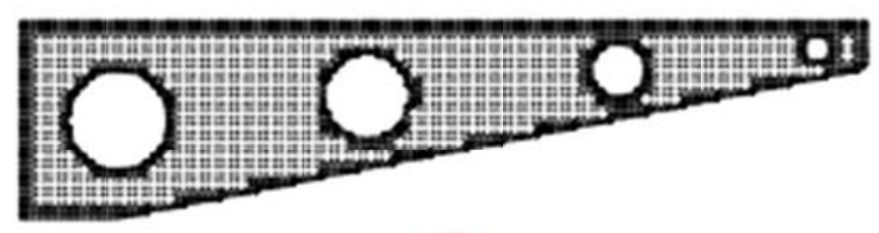

(d)

Figure 3.1: (a) Hierarchical space decomposition is imposed over the geometric domain in Figure 2.8. (b) Basic types of the 2D intersection cases (up to symmetry and rotation transformation). (c) Allocation of the integration points in the integration cells. (d) Positions of the integration points in the geometric domain shown in Figure 2.8. 
The specified values of the convection coe cient, $\alpha$ and ambient temperature, $T_{a m b}$ are $10 \frac{W}{m^{2} K}$ and $20^{\circ} \mathrm{C}$ respectively. The heat conductivity, $\lambda$, of the material (Aluminum) is $200 \frac{W}{m K}$. The SSM solution procedure starts with analyzing the type of the boundary conditions and construction of the solution structure that enforces them. In our case we will represent temperature, $T$, using solution structure (2.11) that satisfies the mixed boundary conditions (3.1). The solution structure (2.11) contains approximate distance fields $\omega_{1}$ and $\omega_{2}$ that vanish on the boundaries where Dirichlet and Robin boundary conditions are prescribed. These functions are constructed using the original geometric representation. In our case $\omega_{1}$ and $\omega_{2}$ are constructed from Boundary Representation of the geometric domain in Figure 2.7(a). Plots in Figures 2.7(b) and (c) present isolines of $\omega_{1}$ and $\omega_{2}$ respectively. The plots in blue illustrates the position of the zero set of the approximate distance fields. Once approximate distance fields and solution structure are constructed, application of Galerkin method results in an algebraic system $A C=B$ in which elements of the matrix $A$ and vector $B$ are computed using expression (2.13). Numerical solution of the algebraic problem delivers numerical values of the degrees of freedom in the solution structure. Finally, the solution, $T$, can be computed and visualized. Figure 2.7(d) illustrates distribution of the computed temperature.

The next example illustrates application of the solution structure method to plane stress analysis problems. Geometry of a cantilever beam, applied load and fixations are shown in Figure 2.8. Geometric boundary shown in red is fixed in both coordinate directions while the green boundary is fixed in the horizontal direction only. To satisfy the kinematic boundary conditions the components of the displacement vector are represented by the following solution structures:

$$
u_{1}=\omega_{1} \sum_{i=1}^{N} C_{i}^{1} \chi_{i} ; \quad u_{2}=\omega_{2} \sum_{i=1}^{N} C_{i}^{2} \chi_{i}
$$


As in the previous example, approximate distance fields $\omega_{1}$ and $\omega_{2}$ are constructed from Boundary Representation of the geometric domain shown in Figure 2.8. Their isolines are shown in Figures 2.9(a) and (b). Plots in blue correspond to the boundaries whose motion is restricted in horizontal and vertical directions. The basis functions $\chi_{i}$ are chosen to be tensor product B-splines defined over $60>30$ uniform Cartesian grid.

Application of the solution method results in numerical values of the degrees of freedom $C_{i}^{1}$ and $C_{i}^{2}$. Substituting them back into solution structures (3.2) we can evaluate and visualize the components of the displacement vector as well as the distribution of the von Mises stress (see plots in Figure 2.10).

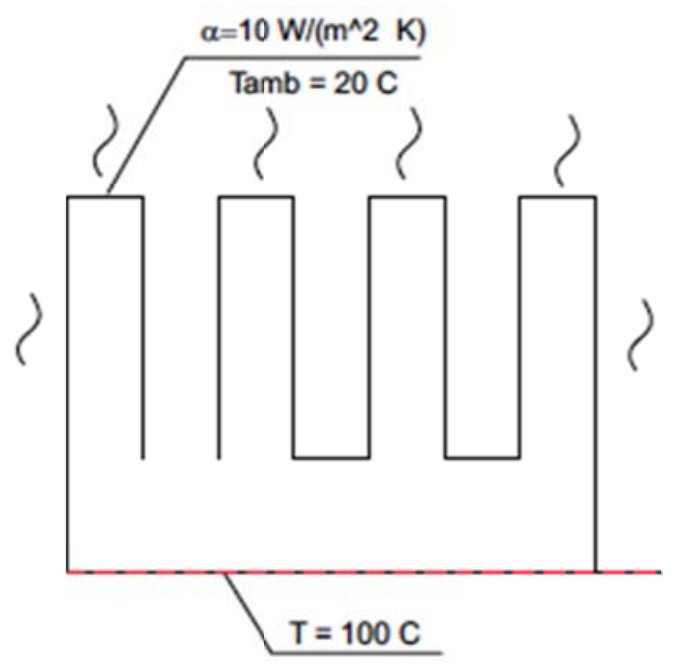

Figure 3.2: A 2D steady-state heat transfer problem with imperfect geometry.

\subsubsection{Integration over domains with geometric imperfections}

In order to place integration nodes in geometrically imprecise models, the PMC values have to be computed at the nodes of hierarchical space decomposition. The presence of gaps and self-intersections of the geometric boundary cause PMC computation to fail. Since traditional methods of repairing geometric models can be computationally expensive, we propose a method of handling geometric imperfections which does not alter the original geometry. In this work, a hybrid geometric model is employed, composed of hierarchical space decomposition, Boundary representation (B-rep) and dis- 


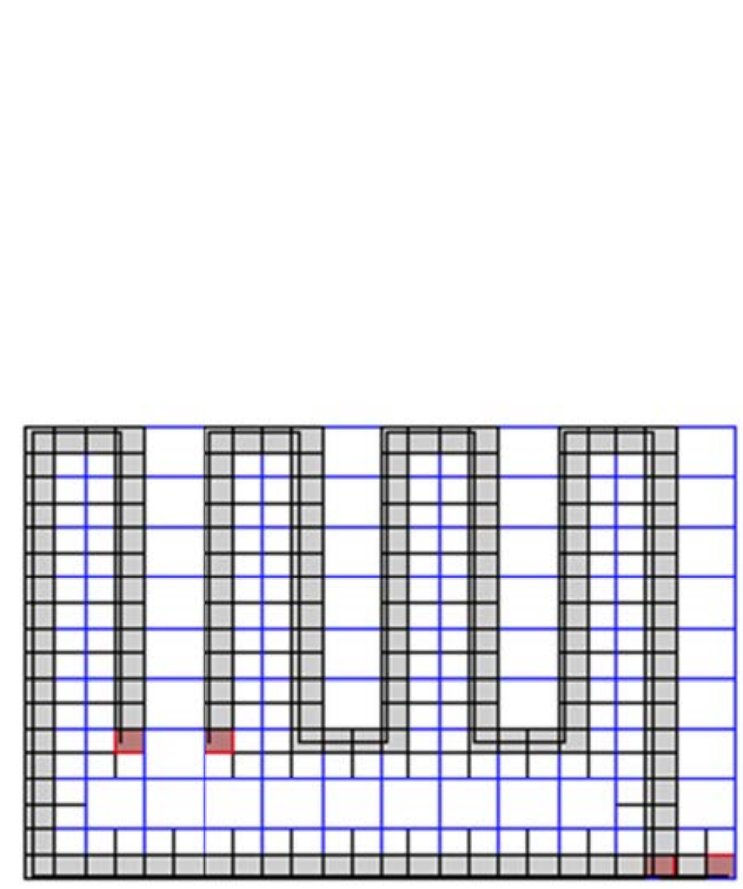

(a)

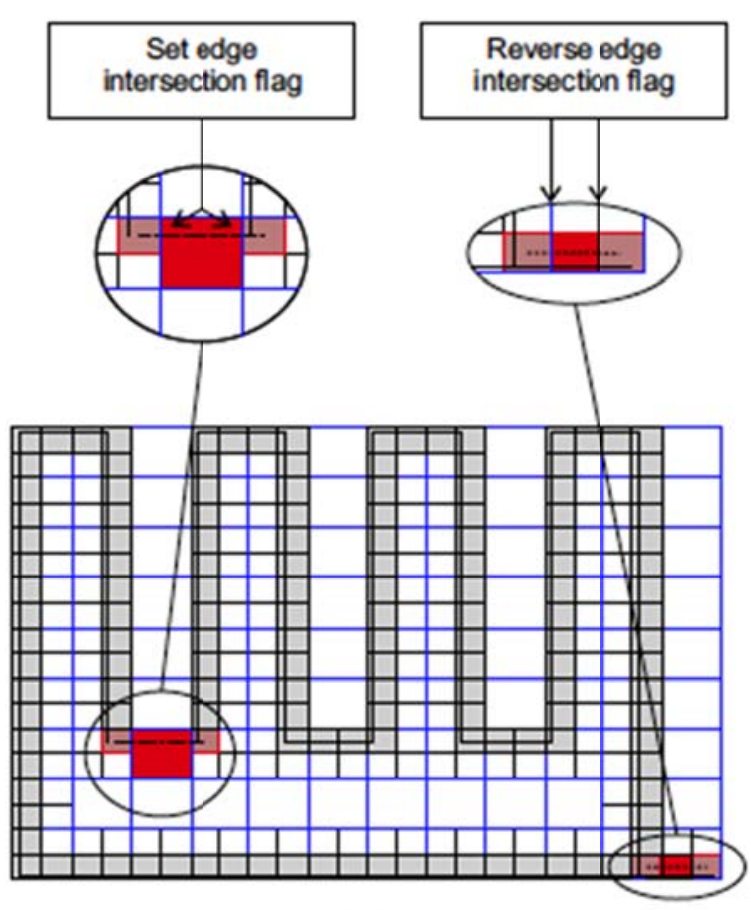

(b)

Figure 3.3: (a) Heatsink with initial Cartesian grid (blue), one application of geometry cell subdivision and marked regular (gray) and irregular geometry cells (light red). (b) Closure of gaps and non-manifold boundary removal using hierarchical space decomposition: closing lines (dashed lines) are converted into the closing cells (dark red). Non-manifold boundaries are removed by reversing the value of the intersection flag for cell edges.

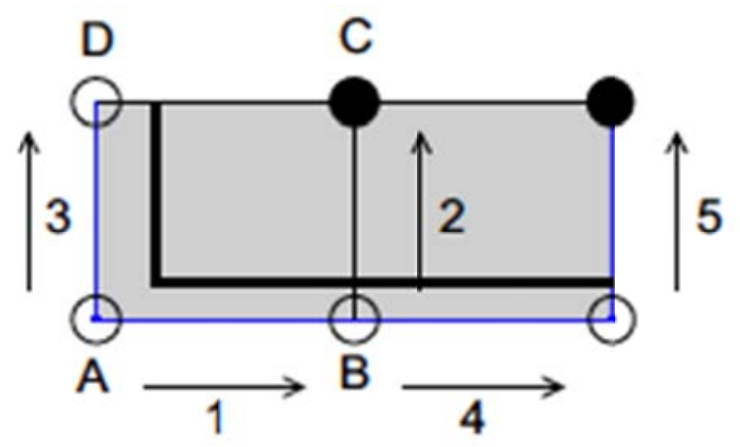




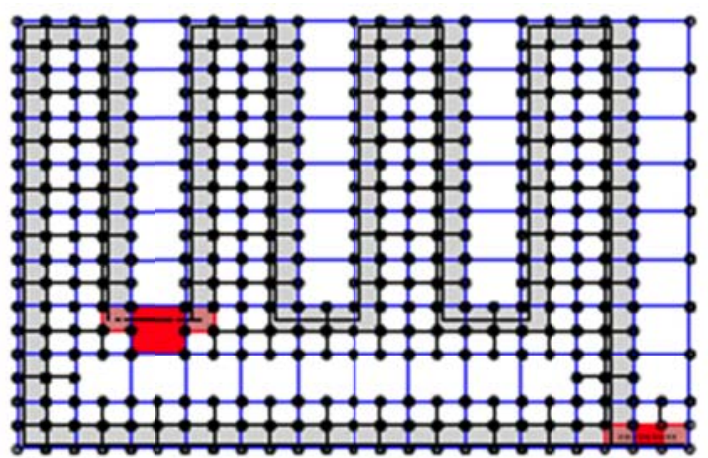

(a)

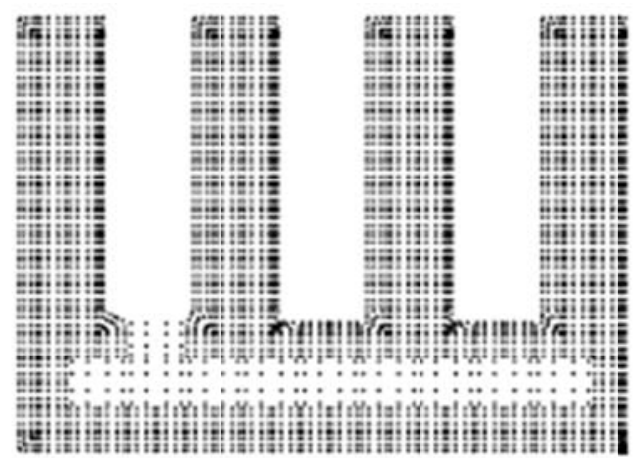

(b)

Figure 3.5: (a) PMC values computed for the geometric model in Figure 3.2. Filled circles designate internal points, while non-filled ones correspond to the points outside the geometric model. (b) Integration points are placed using both original geometric model and closing lines.

tance fields. The idea behind the proposed approach is quite simple. In order to enable PMC computations, the geometric domain needs to be closed in one of these available representations. In our implementation we use hierarchical space decomposition to resolve all geometric inaccuracies, while the original unrepaired model is used later to allocate the integration points.

The integration process over imprecise geometric models starts with the construction of a Cartesian grid inside the bounding box that encloses the geometric model (Figure 3.3(a)). Once the grid is constructed, the geometric boundaries are rasterized (pixelized in 2D and voxelized in 3D) over the grid cells using scan-conversion algorithms [9]. In our data structure the cells which contain or intersect with the boundaries of the geometric model are marked as the geometry cells. In Figure 3.3, geometry cells are shown in gray and red colors. To increase the accuracy of the rasterized representation, the geometry cells can be hierarchically subdivided into subcells. If the original geometric model contains geometric defects or imprecisions, they are also inherited by the rasterized representation. To remove geometric defects and enable PMC computations at the nodes of a hierarchical space decomposition the geometry cells are further classified into regular and irregular cells. The edges of regular cells have an even number of intersections with 
the geometric boundaries. In addition, each edge of a regular cell may not intersect the boundary more than once. These conditions correspond to the continuous boundary — the boundary enter, crosses comes the cell and goes out. Regular cells appear in gray in Figure 3.3. In the cases when cell's edges have multiple intersections with the geometric boundary such cells are hierarchically subdivided until this artifact is removed. Irregular geometry cells (shown in light red in Figure 3.3) mark the locations of geometric defects, such as, for example, gaps and non-manifold boundaries which have an odd number of intersections with the boundary of the geometric model. In general if additional information of the geometry is not supplied we are going to use a straight segment line. After hierarchical space decomposition is built, we cycle through the list of irregular geometry cells and connect each closest-pair with a line, which will be referred to as the closing line. For brevity, we connect the cells center-to-center, though there is no restriction on the end point locations. Once a closing line has been generated, it is rasterized using scan-conversion algorithms on the existing hierarchical space decomposition. All grid cells that intersect the closing line will be referred to as closing cells and the edge intersection flags of these cells are set appropriately. For gaps, the appropriate edge intersection flag is set to true and for self-intersections, the edge intersection flag is reversed (see Figure 3.3(b)). This 2-step process of generate-thenintersect is applied to all pairs of irregular geometry cells. It is important to point out two aspects of this method: first, the original geometric definition remains unchanged and second, each closing line is generated locally in the hierarchical space decomposition and discarded immediately after placement of the integration nodes.

In this study based in models with geometric imperfections we have considered gaps with relative small dimensions. One of the major problems in computational geometry is the closest pair problem, that is based to find a pair of points with the smallest distance between them. It was considered among the major geometric problems at the origins 
of the study of computational complexity of geometric algorithms. Find the shortest distance between two points is reduced to the time required in an Euclidean space and following an algebraic decision tree model of computation. It is possible to compute the distances between all the $n(n-1) / 2$ pairs of points and then pick the pair with the smaller distance. The following references contains well known algorithms commonly

used .[35],[10],[25].

Once the geometric model is closed in the hierarchical space decomposition representation,PMC computation can proceed. It starts from one of the vertices of the bounding box at which the user specifies the PMC value. For definiteness, let us start from the left bottom corner of the bounding box. Also, let us assume that this vertex lies outside of the geometric model. Now we will take a look at a cell of the hierarchical space decomposition that contains that vertex (Figure 3.4). Starting from the point at which the PMC value is known, we visit other vertices of the cell while taking into account whether or not the cell edges intersect the boundary or a closing line. Each edge of a cell reserves an intersection flag in the data structure. It is set when the edge intersects with the geometric boundary or a closing line. For example, the intersection flag for the edge AB (Figure 3.4)is not set. This means that point B has the same PMC value as point A. When the intersection flag is set, like for the edge $\mathrm{BC}$, the PMC values at the end points of the edge have the opposite values. This means that the PMC value at point C should be set to "inside". Similarly, because the edge AD does not intersect, neither geometric boundary nor a closing line, the PMC value at the point D coincides with the PMC value at point A. Once PMC values at all vertices of a cell are determined, they are propagated to the neighboring cells in a similar manner.

Figure 3.5(a) illustrates the final distribution of the PMC values at the nodes of the hierarchical space decomposition.

After the PMC values of all grid nodes have been determined, allocation of integration 
nodes can begin. For internal cells and regular geometry cells, integration nodes are allocated in the same manner as with perfect geometric models. Irregular geometry cells and closing cells can be treated as external cells (no integration nodes), internal cells (filled with integration nodes), or regular geometry cells (partially filled with nodes). In this work, we use the third option. Both the geometry and the closing line(s) are used to allocate integration nodes. In the closing cells, integration nodes are allocated as in regular geometry cells where the "geometry" is the closing line. In the irregular geometry cells, integration nodes are allocated using a line whose end-points are the points where the geometry and closing line intersect the cell. It is important to note that though other end-point combinations are possible, our combination is easily carried into 3D problems without modification.

Figure 3.5(b) illustrates the final placement of the integration nodes. The plot of the resulting temperature distribution is presented in Figure 3.6.

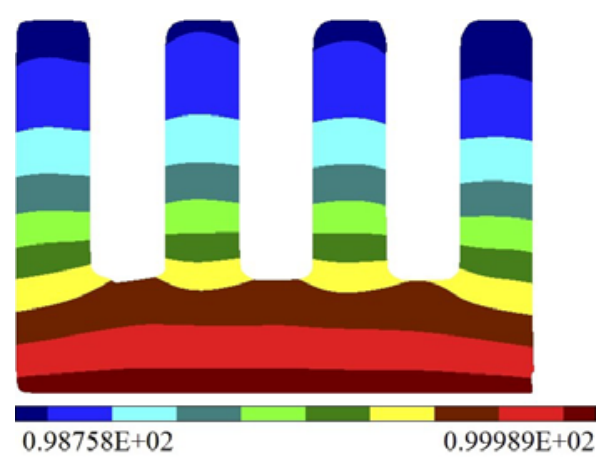

Figure 3.6: Distribution of the temperature field in imprecise geometric model shown in Figure 3.2.

\subsection{Numerical experiments}

The main purpose of the numerical experiments discussed in this Section are to investigate the behavior of the proposed analysis technique in the presence of several di erent types of imperfections as well as the sensitivity of the modeling results to the size of the geometric imperfections. As before, we will limit our discussion to consideration 


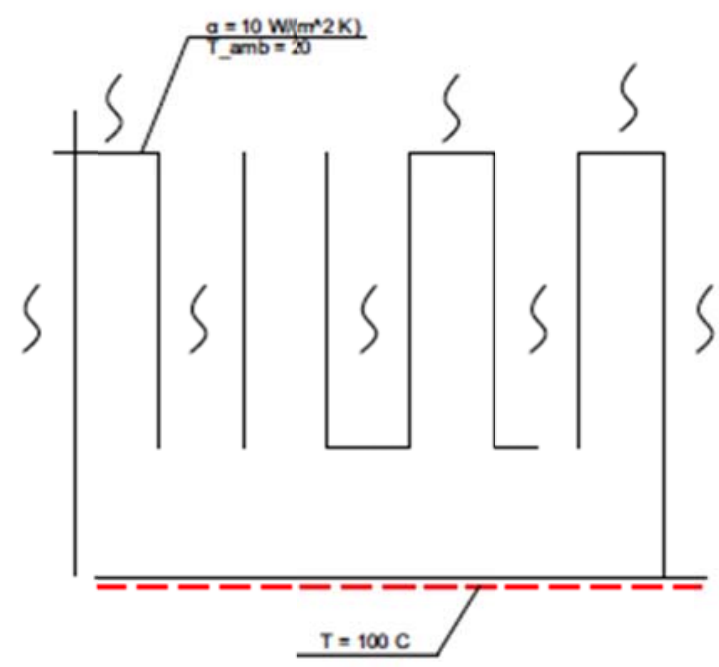

(a)

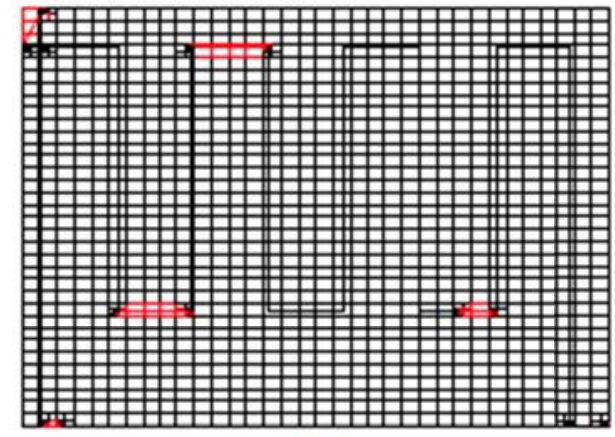

(b)

Figure 3.7: Heat transfer in geometrically imperfect model of a heat sink: (a) geometric model and boundary conditions; and (b) resolution of geometric imperfections on a hierarchical space decomposition.

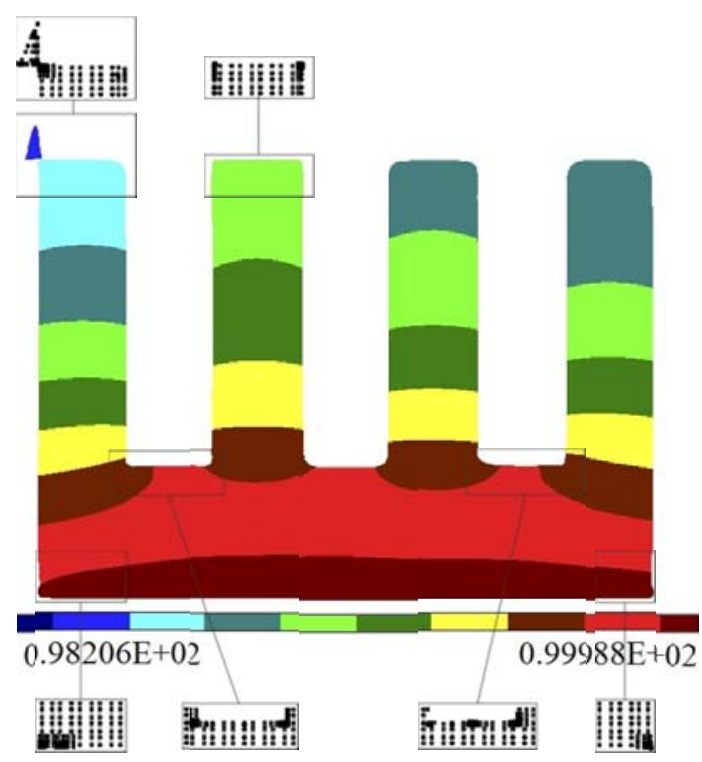

(a)

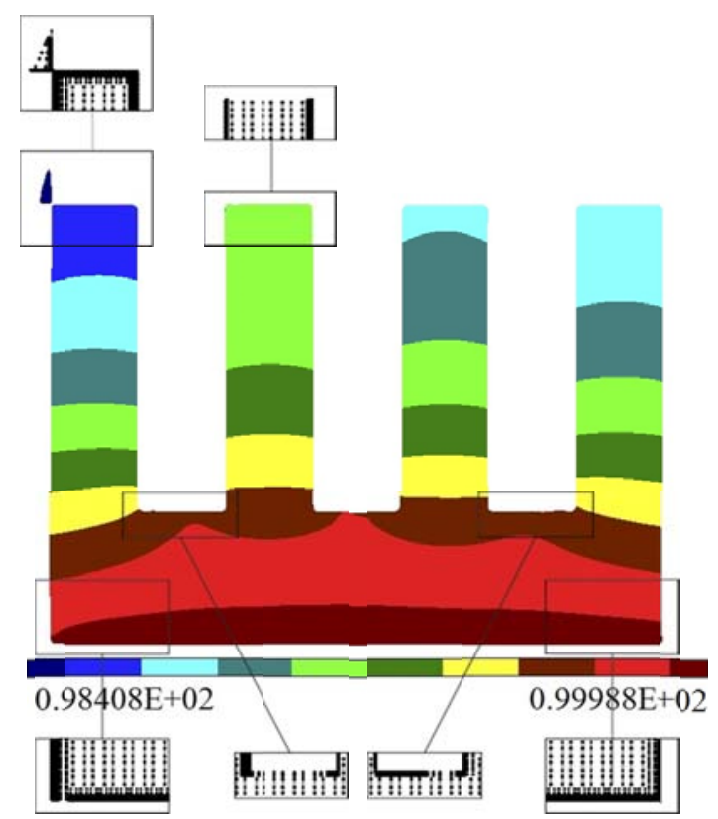

(b)

Figure 3.8: Temperature distributions in imperfect geometric model shown in Figure 3.7(a) obtained using biquadratic B-splines defined over uniform $35>35$ (coarse) Carte- sian grid: (a) without subdividing the geometry cells; (b) with two hierarchical subdi- visions of the geometry cells. 


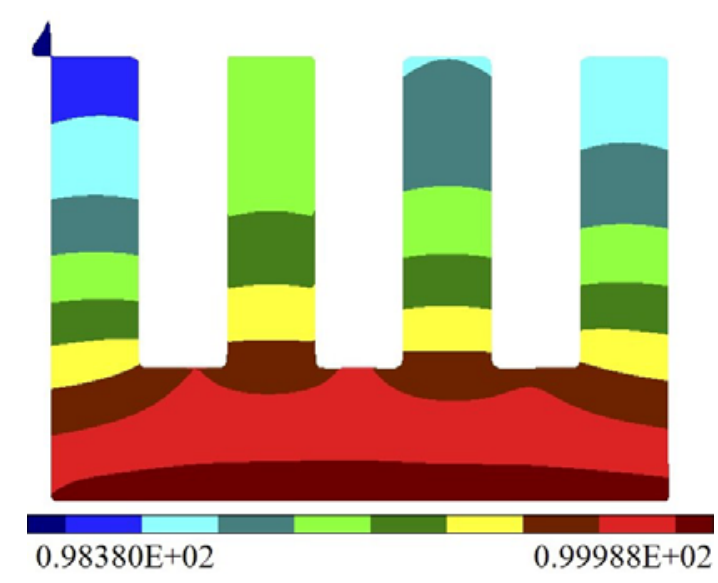

(a)

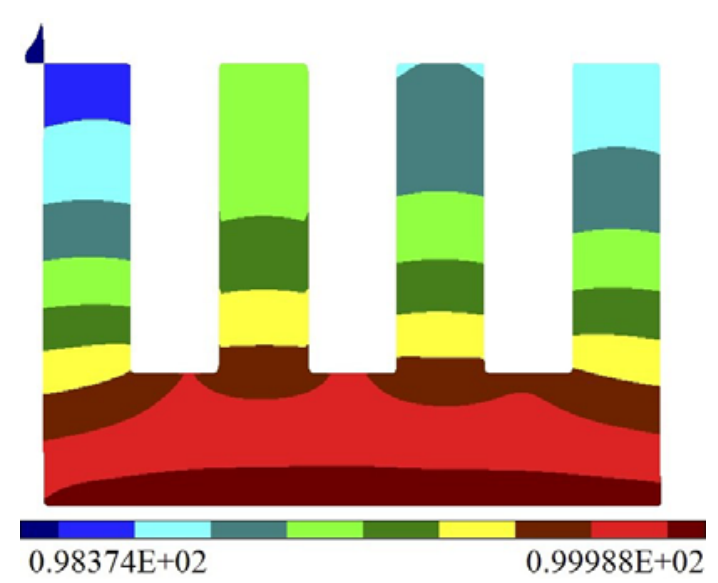

(b)

Figure 3.9: Temperature distributions in imperfect geometric model shown in Figure 3.7(a) obtained using biquadratic B-splines defined over uniform $70 \times 70$ (fine) Cartesian grid: (a) without subdividing the geometry cells; (b) with two hierarchical subdivisions of the geometry cells.

of heat transfer and structural analysis problems.

\subsubsection{Heat conduction in an imperfect geometric model of a heat sink}

Let us start with considering a steady heat transfer problem in the geometric domain shown in Figure 3.7(a). In this example we will use the same boundary conditions and material properties as in the heat transfer example on page 31. This time, however, the geometric model has four gaps of di erent sizes and three non-manifold boundary pieces. Plots in Figures 3.8 and 3.9 illustrate the temperature distributions obtained using the proposed approach. In this numerical experiment coarse $35>35$ (Figure 3.8) and fine $70>70$ (Figure 3.9) Cartesian grids of biquadratic B-splines have been used.The temperature distributions shown in Figures 3.8(a) and 3.9(a) were obtained without hi- erarchical subdivision of the geometry cells. To model the temperature distributions shown in Figures 3.8(b) and 3.9(b) the geometry cells were twice subdivided. Figure

3.7(b) illustrates resolution of the geometric imperfections on a coarse $35>35$ grid. The non-manifold boundary at the bottom-right corner was removed and gaps were closed according to the algorithm we described in Section 3.0.7. The closing lines in 
Figure 3.7(b) are shown with red dashed line. The algorithm, however, did not remove two non-manifold boundaries in the upper-left corner of the geometric model. Instead, irreg- ular cells were connected by a closing line. This happened because the cell containing the intersection point of two boundaries was considered to be a regular cell since it had an even number of intersections with the geometric boundary. Figures 3.8(a) and (b) also illustrate allocation of the integration points near geometric imperfections. As we can see, the original geometric boundaries, as well as the closing lines, participate in the positioning of the integration points. Two levels of hierarchical subdivisions of the geometry cells (Figure 3.8(b)) result in denser cloud of the integration points near original geometric boundaries. Denser grids of basis functions and/or subdivisions of the geometry cells result in converging numerical solutions. Comparison of temperature distribution in Figure 2.7(d) with those in Figures 3.8 and 3.9 reveals a noticeable dif- ference between solutions obtained for perfect and imperfect geometric models. This can be explained by the fact that we assumed the heat insulation on the closing bound- aries. These boundary conditions are natural for Galerkin method and do not require any additional treatment. As we will demonstrate in our next example, as the size of geometric imperfections gets smaller the modeling results converge to the ones obtained in the perfect geometric models.

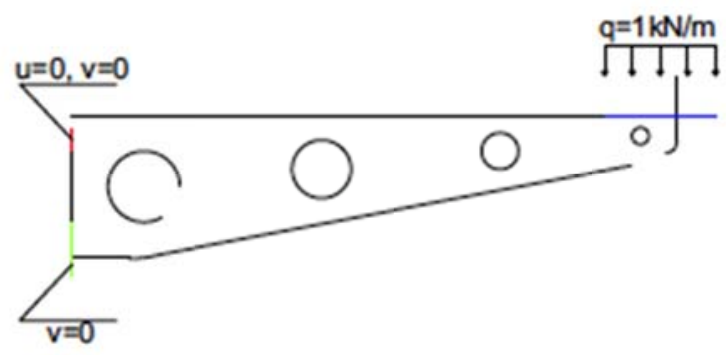


Figure 3.10: 2D cantilever beam with geometric defects.

\subsubsection{D stress analysis in geometrically imperfect cantilever beam}

In this numerical experiment we will investigate behavior of the proposed analysis technique when it is applied to stress analysis problems in imperfect multiple-connected

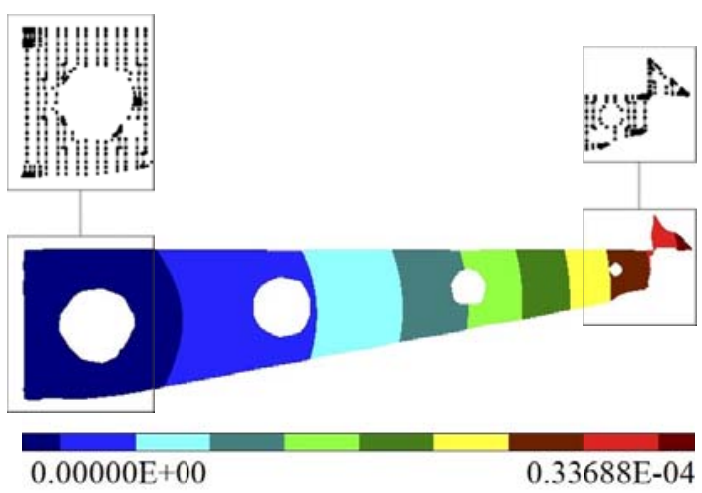

(a)

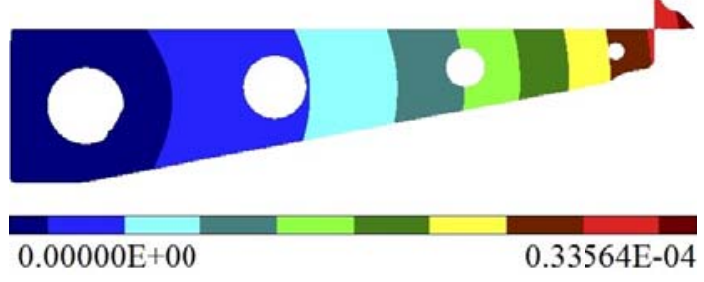

(c)

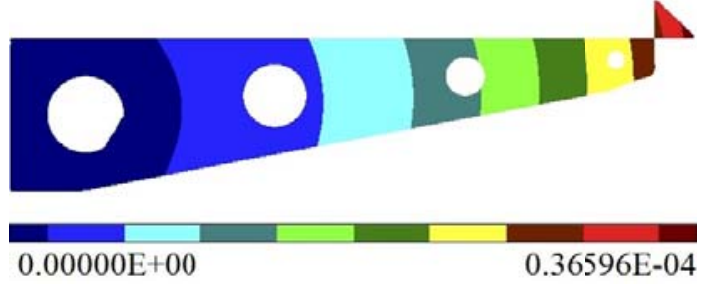

(e)

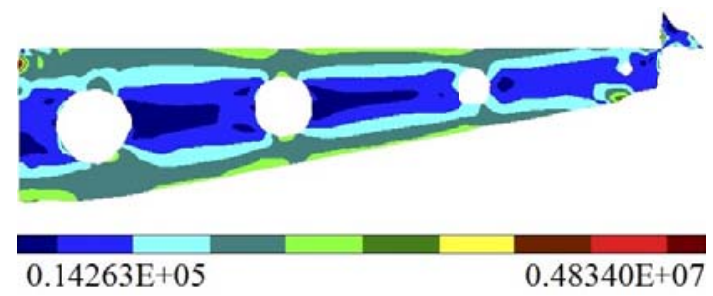

(b)

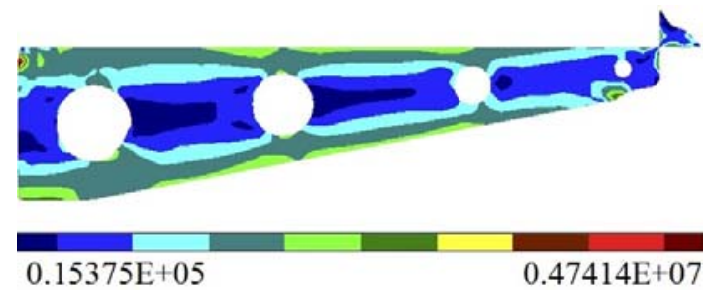

(d)

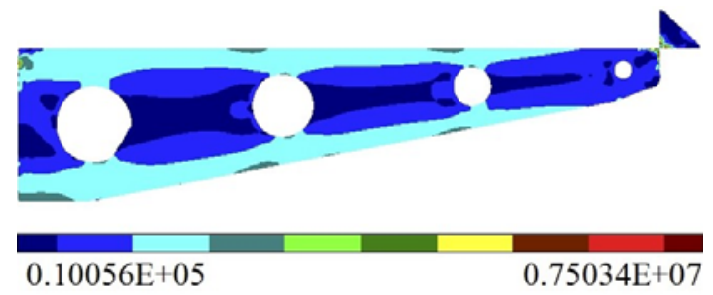

(f)

Figure 3.11: Results of structural analysis of a 2D cantilever beam with large geometric defects: (a) magnitude of the displacement vector and (b) distribution of the von Mises stress obtained using a coarse $35>15$ uniform Cartesian grid of biquadratic B-splines with no subdivision of the geometry cells. (c) Magnitude of the displacement vector and (d) distribution of the von Mises stress obtained using a coarse $35 \times 15$ uniform Cartesian grid of biquadratic B-splines with two hierarchical subdivision of the geometry cells. (e) Magnitude of the displacement vector and (f) distribution of the von Mises stress obtained using a fine $75>25$ uniform Cartesian grid of biquadratic B-splines with two hierarchical subdivision of the geometry cells. 


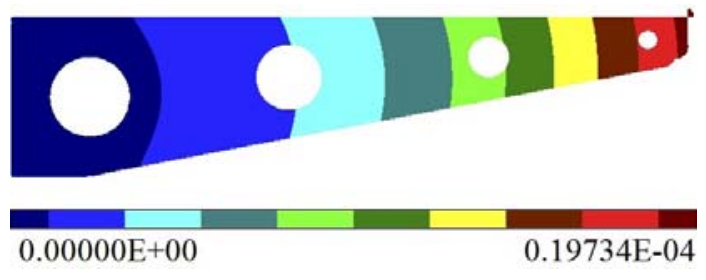

(a)

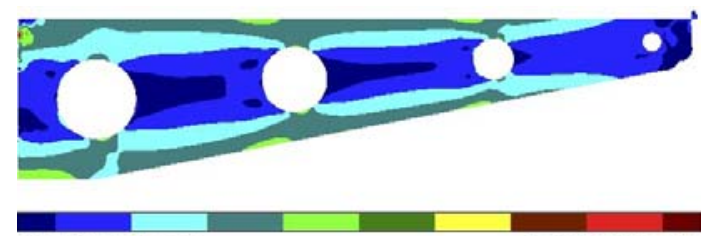

$0.22552 \mathrm{E}+04$
$0.36909 \mathrm{E}+07$

(b)

Figure 3.12: Results of structural analysis of a 2D cantilever beam with small geometric defects: (a) magnitude of the displacement vector and (b) distribution of the von Mises stress obtained using a fine $75>25$ uniform Cartesian grid of biquadratic B-splines with two hierarchical subdivision of the geometry cells.

geometric domain with curvilinear boundaries. We will also explore how the numerical solution depends on the size of the geometric imperfections that include disconnected and non-manifold boundaries.

Figure 3.10 illustrates an imperfect geometric domain with load and fixations applied to its boundaries. In this numerical experiment we used two geometric models with di erent size of the geometric imperfections. The first model exhibits large gaps and dangling boundaries. Distributions of the magnitude of the displacement vector and von Mises stress computed in this geometric domain are presented in Figure 3.11. To perform computations coarse $35 \times 15$ (Figures 3.11(a)-(d)) and fine $75 \times 25$ (Figures 3.11(e)

and (f)) uniform Cartesian grids of biquadratic B-splines have been used. Analysis of the plots in Figure 3.11 reveals that convergence in the displacements is achieved with increase of the grid density and level of hierarchical subdivision of the geometry cells. We can also see that the numerical solution with larger approximation space (Figures 3.11(e) and (f)) provide better approximation of the stresses.

Comparing plots in Figures 3.11, 3.12 and 2.10 we observe that as the size of the geometric imperfections decreases the displacements in imperfect geometric domains converge to those computed in a geometrically perfect domain. Comparisons of the stress distributions illustrate that the stresses are more sensitive to the presence of the geometric 
imperfections since geometric defects play a role in the stress concentrators. However, the presence of geometric defects does not have a global e ect on stress distributions. Stresses demonstrate elevated values locally, near the locations of the geometric imperfections.

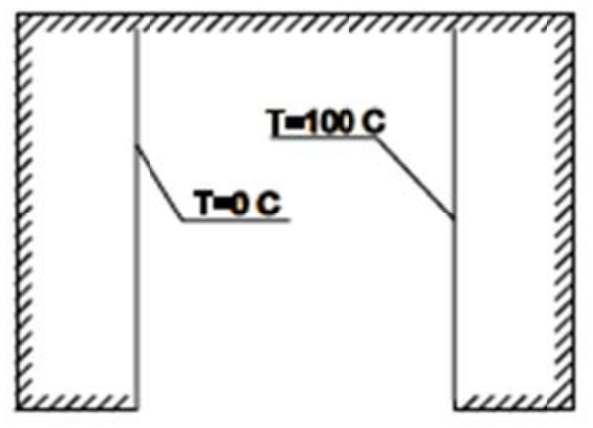

Figure 3.13: Heat transfer in geometrically imperfect model.

\subsection{Conclusions and Future Directions}

The proposed numerical technique enables engineering analysis in imprecise geometric models without applying computationally expensive geometry or mesh repair procedures. The key feature of our approach is a modified geometrically adaptive numerical integration procedure which is based on the ability to compute PMC values at the nodes of hierarchical space decomposition even in the presence of geometrical imperfections and inaccuracies. The developed algorithms use the original geometric representation wherever possible (in regular geometry cells). In irregular geometry cells, integration points are placed based on the location of the original and closing boundaries. Distortions caused by the mismatched original and closing boundaries can be controlled by a proper selection of the subdivision level of the irregular geometry cells. The proposed numerical approach o ers completely automated solution procedure. The implemented algorithms automatically detect geometric imperfections and close the geometric model for the volume integration. The only information the user has to provide is the size of the initial Cartesian grid, which is also used to construct a grid of the B-spline basis functions and subdivision levels for the geometry cells. As an option, 


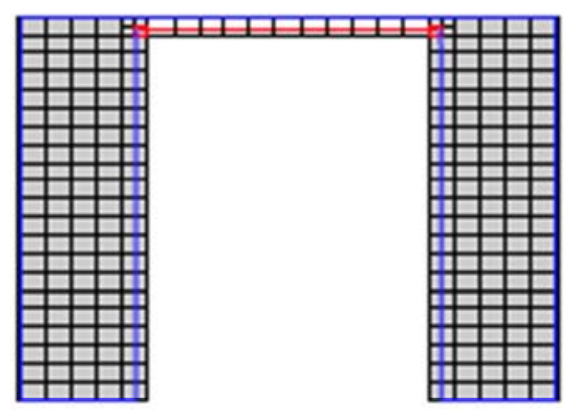

(a)

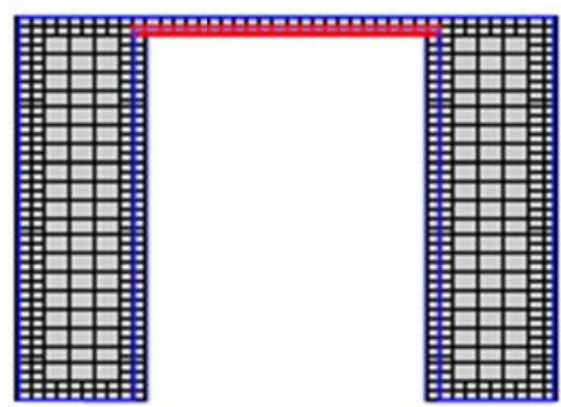

(c)

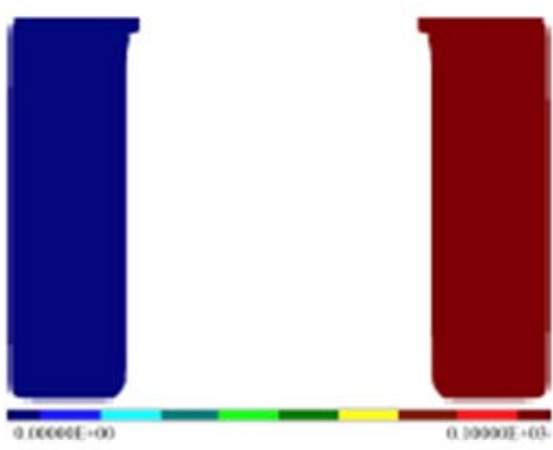

(b)

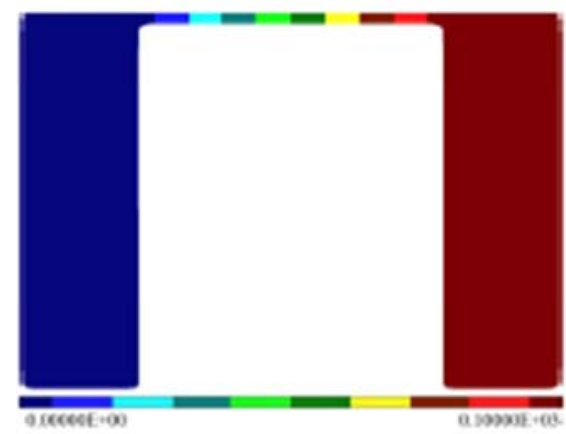

(d)

Figure 3.14: Results of computer simulation of the temperature field in geometric model shown in Figure 3.13: (a) coarse initial integration grid results in relatively large cells that contain the valid geometric boundary as well as the closing line. The latter removes the corresponding integration cells and splits the geometric domain into two parts. The integration cells are shown here in gray color. (b) Temperature distribution obtained using the integration cells in Figure 3.14(a). (c) Hierarchical subdivision of the geometry cells makes it possible to separate the geometric boundary and the closing line. In this case the closing line closes the geometric boundary. The integration cells are shown here in gray color. (d) Temperature distribution obtained using the integration cells in Figure 3.14(c). 


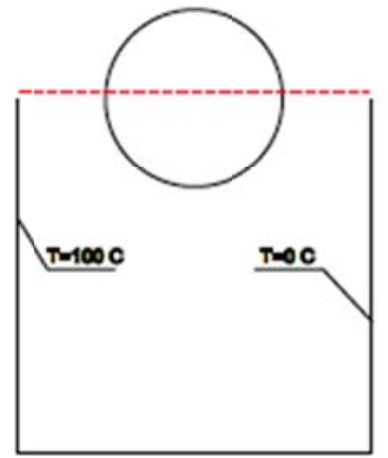

(a)

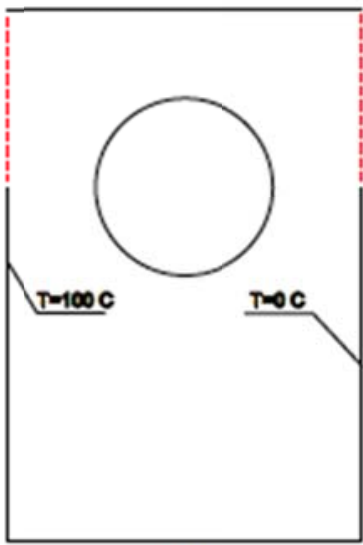

(c)

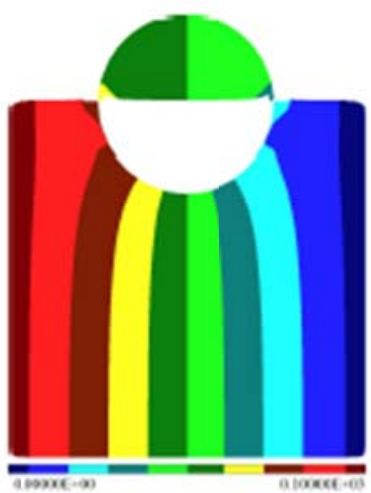

(b)

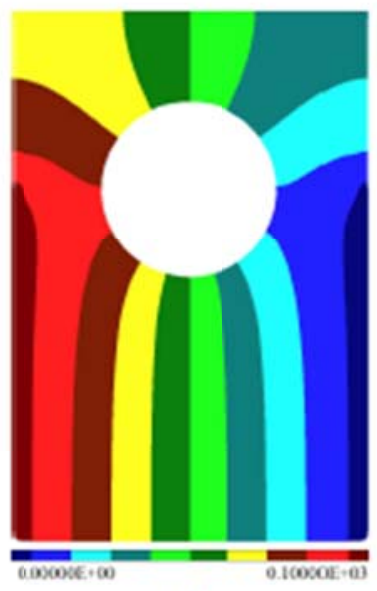

(d)

Figure 3.15: (a) Closing line (shown as a red dashed line) intersects the original geometric boundary. (b) Temperature distribution in the geometric domain in Figure 3.15(a). (c) Adding another boundary piece to the geometric model in Figure 3.15(a) changes the way how the closing lines are created. Now they do not intersect with the original geometric boundaries. (d) Temperature distribution in the geometric domain in Figure 3.15(c). 
di erent subdivision levels of the regular and irregular geometry cells can be specified. Combined with the solution structure method, our numerical technique makes it possible to use spatial meshes that do not necessarily conform to the shape of geometric models while enforcing the prescribed boundary conditions using solution structures as we discussed in

Section 2.2. The meshfree nature of the method and its ability to handle geometric imperfections result in a much higher geometric flexibility in comparison with the traditional analysis tools.

Numerical examples presented in Section 3.1have demonstrated high reliability in handling imperfections in the geometric models. The proposed approach can handle the majority of the real-world imperfections such as gaps, and self-intersecting boundaries, as well as being able to remove non-manifold boundary pieces. Our volume integration algorithms were designed with an assumption that the geometric imperfections are small in comparison with the size of the geometric domain. However, the conducted numerical experiments confirmed that even large geometric imperfections can be handled by our integration algorithms. We would also like to draw attention to a couple of special cases which require close consideration. First, let us take a look at the geometric model in Figure 3.13. This non-closed geometric model results in two irregular cells which are very close to the geometric boundary. The way this geometric imperfection is handled depends on the size of the initial Cartesian grid and the specified level of geometry cells subdivision. If the integration cells are large enough to contain both the boundary of the original geometric domain and the closing line, the latter causes elimination of such cells from the numerical integration. Figure 3.14(a) illustrates the location of the integration cells, original geometry and the closing line. The simulated temperature field is shown in Figure 3.14(b). It demonstrates that the geometric domain was disconnected in two rectangular parts. However, if a finer initial grid is used or if the depth of the geometry 
cells subdivisions is chosen such that the original geometric boundary and the closing line do not appear in the same cells, the integration algorithm closes the gap in the geometry as expected (Figure 3.14(c)), and the resulting temperature field is presented in Figure 3.14(d).

Figure 3.15(a) illustrates another special case when the closing line intersect with the boundary of the original geometric model. This case contradicts our initial assumption about the small size of geometric imperfections and, if processed, may lead to unexpected results, as shown in Figure 3.15(b). Cases such as this one, however, can be easily detected by testing whether the closing boundaries intersect with the boundary of the original geometric model. If the intersection is detected, a warning message is generated. Then the user has either to accept the modeling results (if they have a sense) or modify the geometric model in a such way that the closing boundaries do not intersect with the original geometric boundaries as it is shown in Figure 3.15(c). Figure 3.15(d) presents distribution of the corresponding temperature field.

The proposed algorithm removes non-manifold boundaries if there is only one nonmanifold boundary piece is attached to a corner of a geometric model. In the case of two or more non-manifold boundaries attached to the model's boundary at the same point (see Figures 3.7(a) and 3.16(a)), the proposed method creates an "attachment” that can clearly be observed in Figure 3.8. Creation of such attachment can be prevented if two additional irregular geometry cells are placed in the cell which has four edge-boundary intersections as illustrated in Figure 3.16(b). The closing lines connect irregular cells with one edge-boundary intersection with the irregular cells with four edge-boundary intersections. The closing lines passing through the cells which contain the non-manifold boundary pieces reverse intersection flags on the cells edges. As a result the non-manifold boundary pieces are removed and an attachment is not created (Figure 3.16(c)). When a simulation of the physical fields in imprecise geometric domains is performed, the 


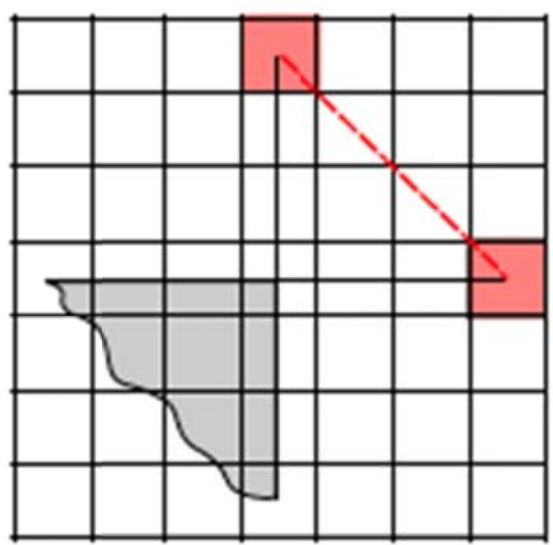

(a)

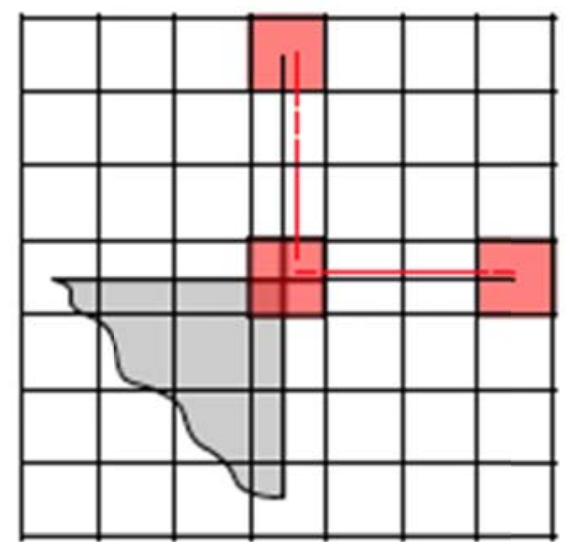

(b)

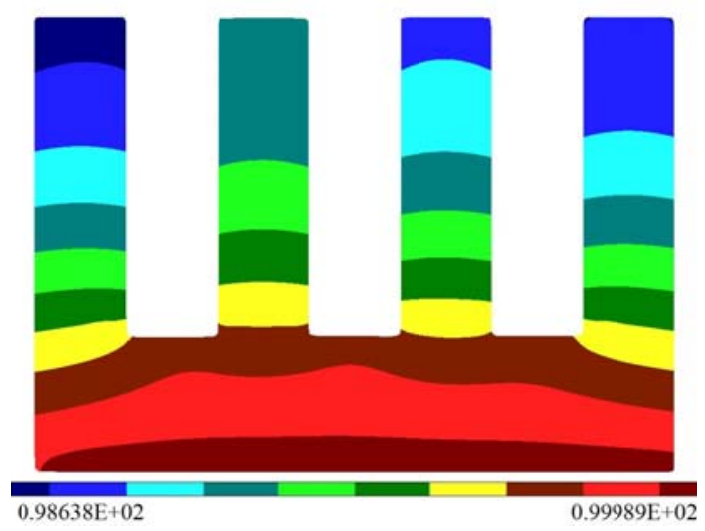

(c)

Figure 3.16: Resolution of non-manifold boundaries: (a) placement of two irregular cells resolves non-manifold boundaries but creates an "attachment" to the geometric model. (b) By placing two additional irregular cells in the cell which has four edge-boundary intersections non-manifold boundary pieces can be removed. (c) Simulated temperature distribution with removal of "attachments". 
validity of the computational results must be ensured. Our preliminary results suggest that in most cases the computation error introduced by the imperfections gets smaller when the size of the geometric imperfections decreases. In analyzing the result of our numerical experiments, we also noticed that the di erence between solutions obtained in valid and imprecise geometric models are caused by the boundary conditions assumed for the closing boundaries. In our numerical examples we used default natural boundary conditions that correspond to heat insulation in the case of heat transfer problems, and to a traction free boundary in the case of structural analysis problems. 


\section{CHAPTER 4}

\section{EXTENSION INTO 3D: 2D PARALLEL SLICES}

A 2D parallel slices approach to simulate 3D geometrical models is proposed following the well known method used in the medical procedure called the CT (Computerized Tomography) scan which was created to visualize the existence of tumors in an human body specifically in brain and breast tumor recognition. The proposed approach consists of parallel layers (2D) built up with the 3D imprecise solid model placed on it. The proposed method uses solution structures as well as those that combine the distance fields to the geometric boundaries and basis functions to enforce the prescribed boundary conditions. Figures 1.3 (b) and (c) illustrate allocation of the integration points in 2D imperfect geometric domain shown in Figure 1.3 (b) using our new approach.

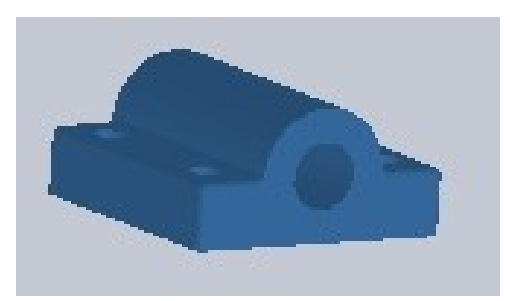

(a)

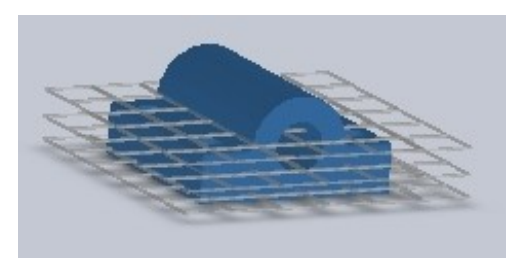

(b)

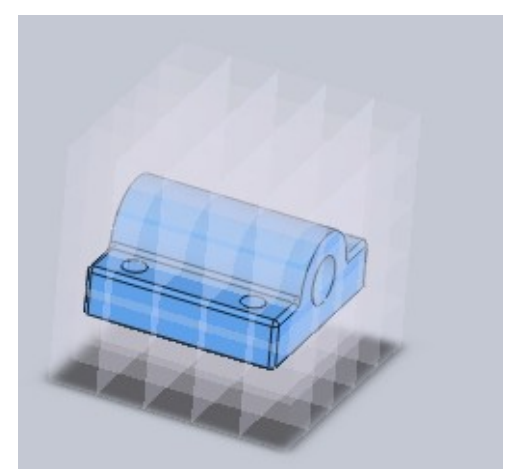

(c)

Figure 4.1: (a) Imprecise solid model. (b) 3D numerical integration can be performed using 2D integration technique applied to the Cartesian parallel cross sections of the geometric model. (c) 3D hierarchical space decomposition allow to allocate integration nodes that can be used to perform numerical integration in geometrically imprecise domains.

Applying the same methodology for 2D Boundaries representations (B-rep) using the same hierarchical space decomposition to resolve geometric inaccuracies in 2D by applying a geometrical adaptive integration over the geometrical model creates and allocates layers from the bottom to the top. The 3D geometrical model sliced in finite parallel Cartesian grid layers, each one treated following the same 2D approach, means 
using a composite geometric representation in layers with geometric imperfection. Using a hierarchical space decomposition method, as proposed in $2 \mathrm{D}$, resolves the geometric gaps and inaccuracies without repair or alteration of the original geometry and allows us to allocate integration points in the original model. Integration gauss allocation process in $3 \mathrm{D}$ will be applied using the 2D constructed Cartesian grids with gauss allocation points, once the PMC values of all grid nodes have been evaluated. For internal cells and regular geometry, the integration nodes will be allocated in the same manner as perfect geometric models. Irregular geometry cells treated as the regular geometry cells, partially filled with nodes, means that both the geometry and the closing line(s) are used to allocate integration nodes. The rule for allocation gauss nodes for imperfect geometries in $3 \mathrm{D}$ follow the same algorithm and procedure used for $2 \mathrm{D}$ but each slice has its own weight following the gauss integration rule. The geometric boundaries are voxelized over the grid using scan conversion algorithms.

The meshfree method uses distance fields to construct functions and data structure for the integration at running time. Allocation of integration points in 3D using adaptive flexible integration algorithms over the original imperfect geometry has achieved satisfactory numerical results using the parallel slices technique. As mentioned before, the application of a geometrically adaptive integration over the 2D parallel layers allows the performance of computational experiments in real time with results that converge to the exact solution with conservative running cost. The algorithm to analyze imperfect geometries described in previous chapters relies on hierarchical space partitioning similar to Quad/Octree decomposition that allows the application of PMC in the entire geometry. The same hierarchical decomposition will be used to allocate gauss integration points even in areas where the geometry has gaps or imperfections. The following chapters are dedicated to validation and verification of experiments that will show the approximation to the exact solution using di erent numbers of gauss allocation points. 
A mathematical expression for volume integration, as an example, can be represented as a weighted sum of the values for each integrand at the integration nodes.

$$
\begin{gathered}
\iint_{\Omega} \int_{\Omega} f(x, y, z) d \Omega=\sum_{i} \sum_{j} \sum_{k} W_{i} W_{j} W_{k} f\left(x_{i}, y_{j}, z_{k}\right) \\
\iiint_{\Omega} f(x, y, z) d \Omega=\sum_{k} W_{k}\left(\sum_{i} \sum_{j} W_{i} W_{j} f\left(x_{i}, y_{j}, z_{k}\right)\right)
\end{gathered}
$$

A 3D integration points approach will be proposed using an extension of the 2D geometrically adaptive integration over domains with geometric imperfections. Using and regrouping terms from a lattice rule, a 3D solid model can be subdivided into 2D parallel slices. Every slice will be treated for the 2D grid allocating integration points for 2D (plane $x, y$ ) and every 2D slice will has its own weight to fulfill the gauss integration rule. The formula above shows the lattice rule procedure and the Figure 1.3 (b) shows a 3D solid model inside the slices to enforce the governing equation and to fulfill the boundaries conditions imposed. The following algorithm is proposed to integrate 3D allocation points in imperfect geometric models without incurred in expensive geometric healing and repair.The same Closing Gap method proposed in 2D is going to be applied at every 2D discretized slices. The following algorithm obtain the value of the volumetric integral of a model in 3D with geometric imperfection:

1. Step 1 Set inputs $=\mathrm{f}(\mathrm{x}, \mathrm{y}, \mathrm{z}) ;(X \min , Y \min , Z \min , X \max . Y \max , Z \max ) ;\left(n_{x}, n_{y}, n_{z}\right)$; $n_{(\text {gauss })}$

2. Step 2 For $\mathbf{z}=n_{z}=1$ to Zmax ; Slices $2 \mathrm{D}$ Allocation;

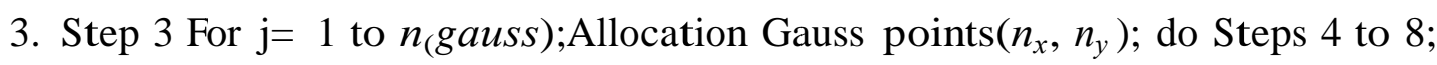

4. Step 4 Build Binary Tree $->(P M C)$; do(Xmin,Ymin)to(Xmax,Ymax)

5. Step 5 IF(function(PMC) Bad Cell Found)do Step 6; 
6. Step 6 Ray Intersect function — > (function(CG)Closing Gap) do; Step 8

7. Step 7 Else;

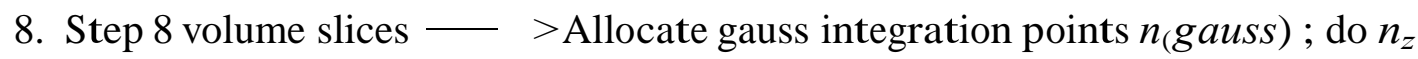
((Xmin,Ymin) to ( $\mathrm{Xmax}, \mathrm{Ymax})$ );

9. Step 9 Output Get Integral Value $->($ Evaluation $f(x, y, z)$ of the integrand func- tion)

The algorithm described before shows a reliable way to compute and analyze mechanical models with imperfections without repair or any other geometric healing method. The algorithm requires the feed of four important inputs. The input 1 is the integrand which represent in this sample the value of the volumetric integral. The integrand represent the value of the weighted expansion series of basis functions belonging to the solution structure for any boundary problem proposed. Inputs 2 and 3 define the bounding box which will contain the model under study. Will be set nx, ny, nz as the number of the initial grid cells and $\mathrm{n}$ is the number of gauss points, the algorithm use the same number of gauss points (n) for each coordinate direction. Numerical experiments will be processes having di erent size grids and di erent number of allocation gauss points to validate convergence. It will show graphically the convergence when compared with the exact solution either for perfect or imperfect models. Step 4 will generate a binary tree for cells identification. Slices 2D grid generation $\left(d_{x}, d_{y}\right)$; are allocated in parallel to cover the entire model. A For Loop iteration( 6 to 8) create a hierarchical 2D space decomposition to be imposed over the geometric domain. The integration process starts with the cartesian grid already placed over the geometric model; the grid is built up with the cells aligned with the support of the basis functions(as fig 4.1.b)and classified following its relative position to the geometric model imposed. Cells can be internal, external and boundary cells. Once the classification process is done and 
the process to place integration nodes start; a Point Membership Classification (PMC) function must be compute since is necessary to obtain the relative position of a given point in the space with respect to the geometric model; this function allows to know if the point is inside or outside of the domain. The presence of gaps and self intersections of the geometric boundary cause PMC computation to fail. The integration process over imprecise geometric model to compute PMC must be applied following the steps already explained. Once the integration points are allocated in the $2 \mathrm{D}$ cartesian grids a lattice rule for 3D domains is applied and compute. The loop leads to end and discrete sum functions are added to conform the final value of the integrand. 


\section{CHAPTER 5}

\section{VALIDATION AND VERIFICATION OF THE PROPOSED METHODOLOGY}

The goal of this chapter is to investigate accuracy, convergence and stability of the proposed numerical integration algorithms. To do this we will perform two series of numerical experiments. In the first series, we will apply the developed algorithms to volume computation of precise and imprecise CAD models. This will allow us to investigate numerical properties of the proposed approach and compare the results for precise and imprecise models. For our experiments we have selected CAD models that have no geometric defects such as gaps in the boundary, sleeve boundaries, etc. To create imprecise geometric models we will convert the selected CAD models into STL format which represents the geometric boundary using triangular tessellation. The fact that the data in STL format can be stored in a text file makes it very convenient for modifying the geometry and introduces various geometric imprecision. The second series of numerical experiments aims at applicability of the proposed approach to model physical processes in geometrically imprecise models. We will observe and investigate how accuracy, convergence and the stability of the algorithm depend on the density of the initial Cartesian grid and the number of the Gauss integration points.

\subsection{Volume Computation in Precise and Imprecise CAD Models}

In this series of numerical experiments we will use CAD models originally created in the SOLIDWORKS software package. SOLIDWORKS has the tool package to calculate the volume of the CAD models, so we will use the values provided by SOLIDWORKS as a base line for our numerical experiments.

The geometric engine which we interfaced our numerical algorithms can import geometric models in STEP and STL formats. We will use STEP format to represent perfect geometric models, while Standard Tessellation Language (STL) format will be used to 
represent imprecise geometric models. STL is used to describe a raw surfaces triangulation and can be saved in a text file that can be easily modified in any text editor. To introduce imperfections in our geometric models we will remove or modify some boundary triangles in the STL file.

To investigate the convergence and stability of the proposed algorithms for each geometric model we will perform experiments where:

- Will be used the same number of Gauss points (3) allocated in each coordinate direction, while the density of the grids will be changed. The following initial grids will be used: 10x10,20x20,30x30,40x40,50x50,60x60,80x80 and 100x100.

- Using the same initial 3D grid 20x20x20 we will change the number of Gauss integration points in each coordinate direction. In our experiments we will use 2, 5, 7 and 9 integration points.

The results of each numerical experiment will be presented on the following plots:

- Computed volume vs grid size.

- Integration error vs grid size.

- Computed volume vs number of Gauss points.

- Integration error vs number of Gauss points.

The integration error measures the di erence between the volume computed using the proposed slicing algorithms and the value given by SOLIDWORKS. Before starting the experiments on di erent precise and imprecise models we will expose the incapacity of the modeler package mentioned when an imprecise model is used. The following two figures show both models under the simulation package. represents our model for a precise bracket geometry. The SOLIDWORKS package is ready to process the geometric model without questioning any mistake or design error. The Figure 5.2 represents 


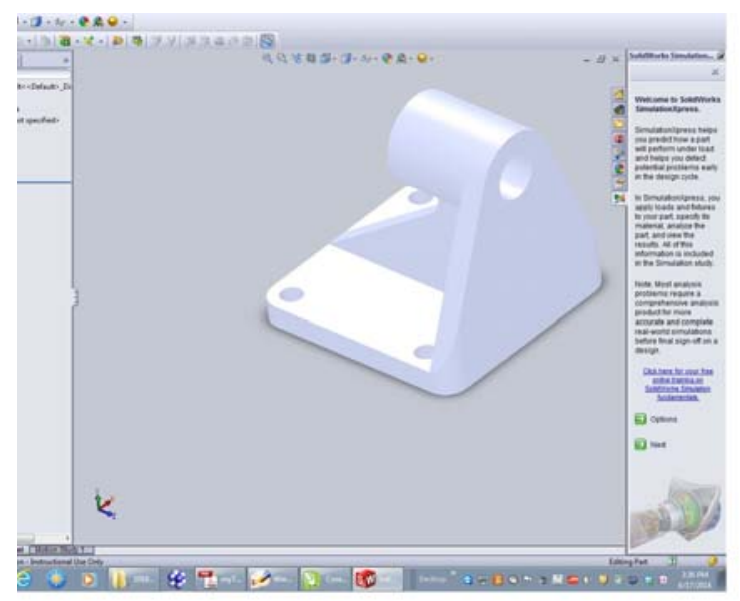

Figure 5.1: Precise bracket model.

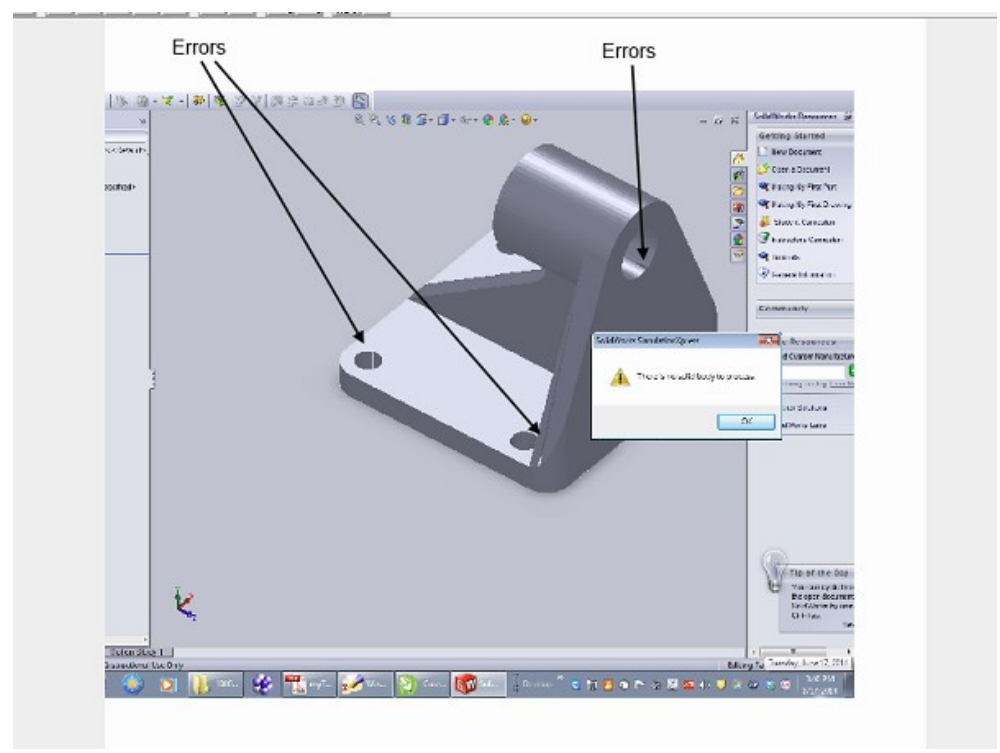

Figure 5.2: Imprecise bracket model.

the same precedent model but with imprecise geometries. It shows how the SOLIDWORKS package stops running claiming a damaged model and no possible simulation performance. The SOLIDWORKS analysis package releases a typical comment such as, 
"There is no solid body to process.” We have indicated also in this figure the location of three di erent errors, manually created using STL file geometry. In a real case when a designer starts modeling a solid, the solid construction system uses a logic union and intersection geometries which are subjected to round 0 and finite precision that could create gaps and overlapping lines.

\subsubsection{Numerical experiment 1: Volume Computation of a Bracket}

The first set of numerical experiments is based to compute the volume value of a bracket, cylinder, and a spur gear with precise and imprecise geometries. The models were originated using SOLIDWORKS and saved in STEP and STL extension files respectively. Four (4) di erent kinds of plots were generated to investigate convergence and stability as mentioned already in paragraphs before.

For bracket model with precise geometry:

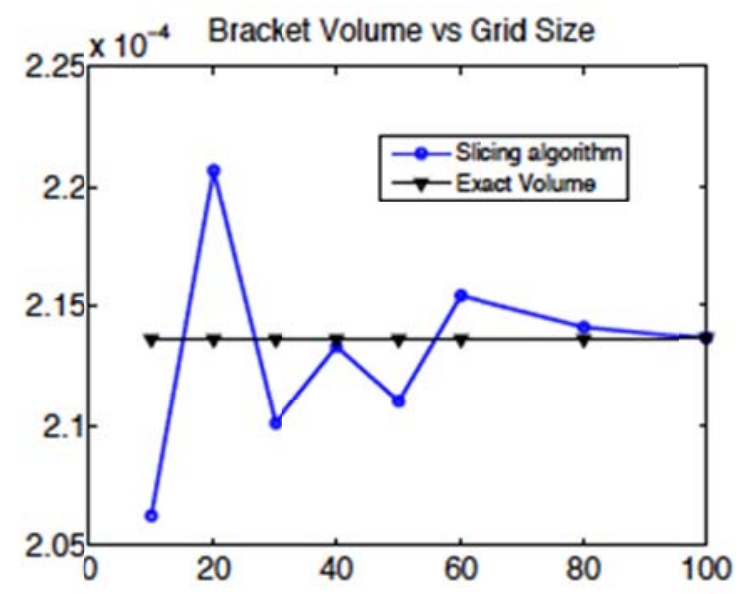

Figure 5.3: Precise bracket volume vs grid size.

Figure 5.3; shows a fast convergence of the computed volume to the real value when increasing the grid cells from $40 \times 40$ to $100 \times 100$. The allocation of only 3 gauss points allowed to obtain a satisfactory result even having some alternate value around the exact volume value. 


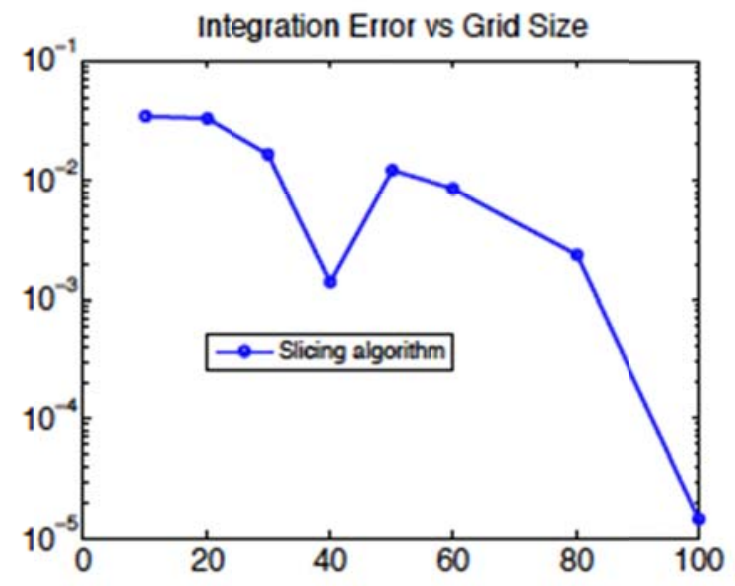

Figure 5.4: Integration errors vs grid size.

Figure 5.4 shows the decrease of experimental errors when increasing the grid density; at 40 slices is notable a local variation of the convergence path. Integration errors decrease using 50 to 80 slices and finally stabilized to a lower level value at 100 slices.

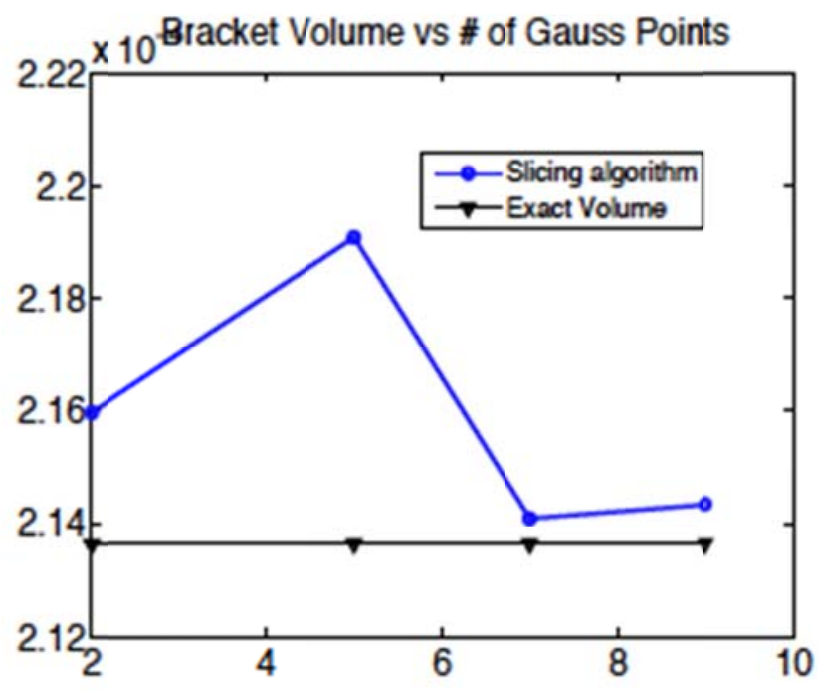

Figure 5.5: Precise bracket volume vs gauss points.

Figure 5.5 refers to the change of volume evaluation depending of the number of Gauss points allocated. Keeping the same 3D grid of 20x20 the first gauss points allocation from 2 to 5 do not converge to a satisfactory result; once the numbers of gauss points allocated are over 5 points the fast approximation to the exact volume value is reach it 


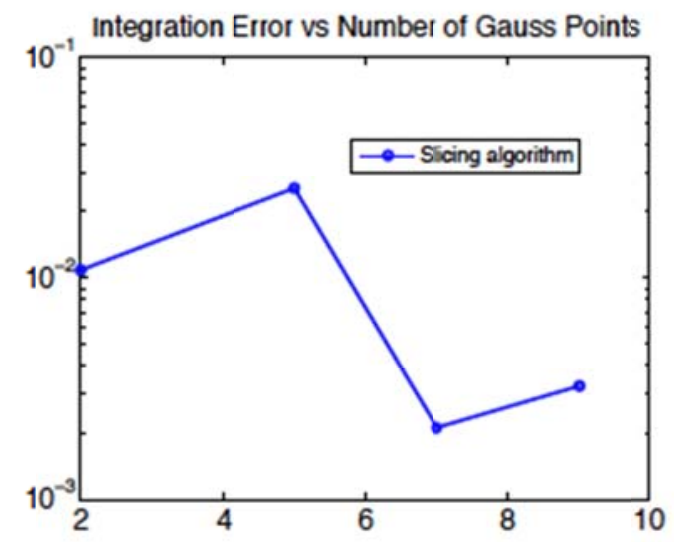

Figure 5.6: Integration error vs number of gauss points.

The same interpretation of the previous experiment can be applied for the Figure 5.6. Errors decrease substantially after the number of allocation points are more than 5 points. For this particular experiment the best result can be obtained over 7 allocation points . The use of a 20x20 parallel grids limit the possibility to obtain better result at smaller number of gauss points.

For bracket model with imprecise geometry:

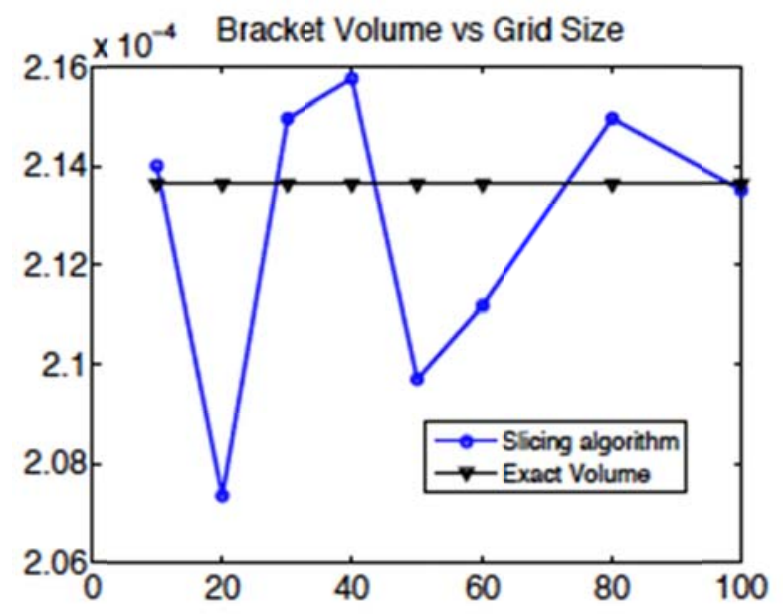

Figure 5.7: Imprecise bracket volume vs grid size.

For this model with geometric imperfection Figure 5.7 the integration volume value is very fluctuant. However its convergence improve notably as the grid size increase. Its reach a perfect convergence at 100x100 grid size and using 3 integration gauss points; 
reaching the same numerical approximation when using a perfect geometric model

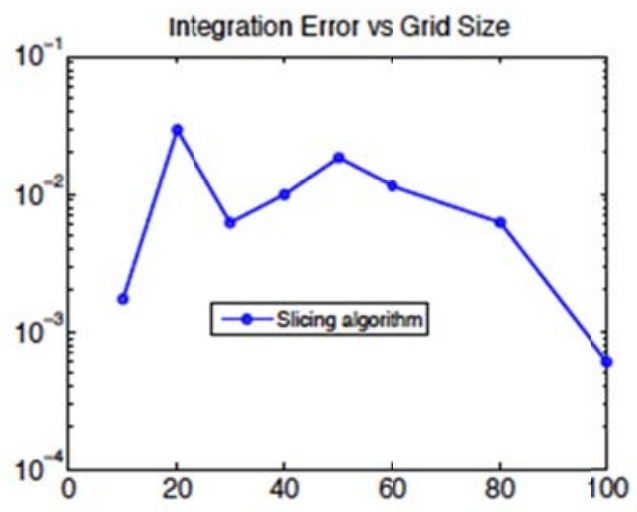

Figure 5.8: Integration errors vs grid size.

Figure 5.8 shows that between 20 and 50 slices the convergence vary but has an good convergence after placing larger slices grids. At 100 x100 grids the errors are lower than the perfect geometry evaluated with the same number of slices. This experiment in particular allow to understand the meaning of the algorithm designed to evaluate PMC in imperfect geometries. Increasing the grid slices in 2D, improve notably the results even using a lower number of gauss integration points with an acceptable computation time for 3D models.

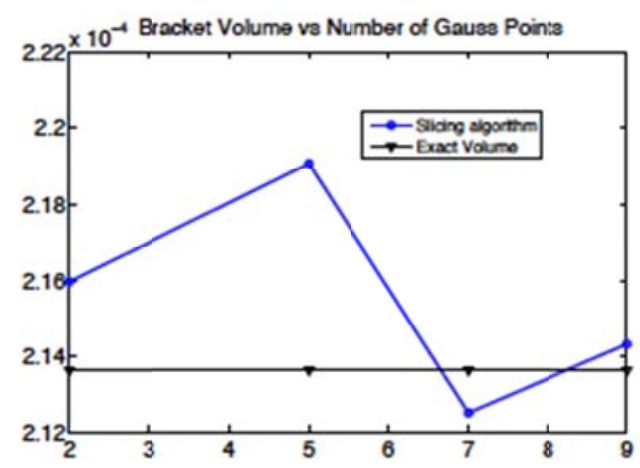

Figure 5.9: Imprecise bracket volume vs gauss points.

Figure 5.9 shows as similar result as the perfect geometry case; a larger number of integration gauss points, drive a better numerical convergence. The interpretation of 
this experiment using di erent number of gauss points and using just a 20x20 grid give a complete understanding of the importance of the number of parallel grids we have to use for the integration scope and the number of gauss points to allocate in order to obtain an acceptable result .

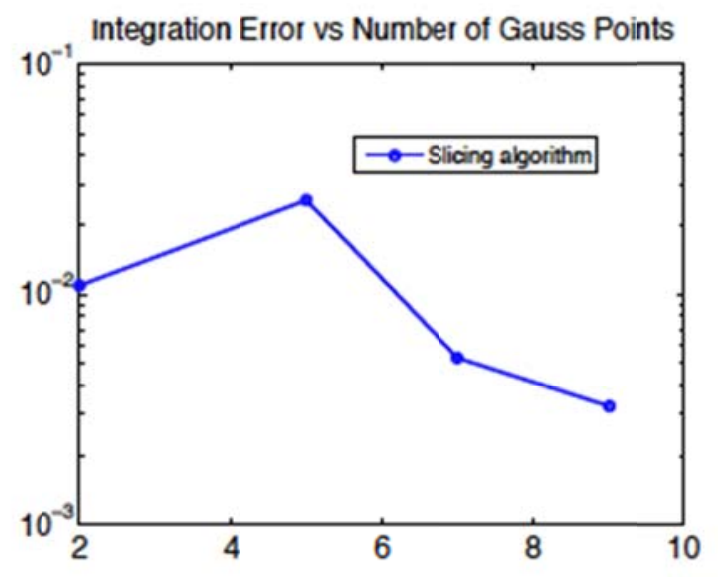

Figure 5.10: Integration error vs number of gauss points.

Figure 5.10 as mentioned before;using a limited quantities of parallel slices ;in this case $20 x 20$ is necessary to increase the number of gauss points to have a better convergence and numerical approximation, for this particular experiment the allocation of more than 6 integration gauss points allow a better reduction of the integration errors.

\subsubsection{Numerical experiment 2: 3D Simulation in a Cylinder}

For Cylinder model with precise geometry:

The Figure 5.11 represent our model for a precise cylinder geometry.

Figure 5.12 Shows a rapid convergence of the slicing volume algorithm to the exact value using 3 integration gauss points. From 20x20 slices grid to 100x100 the value of the computed volume, lightly vary around the exact value to converge satisfactorily at 100x100 grid density.

Figure 5.13 can be interpreted as a continuation of the result obtained and showed in the previous plot. Integration error are evaluated as a di erence between the real value 


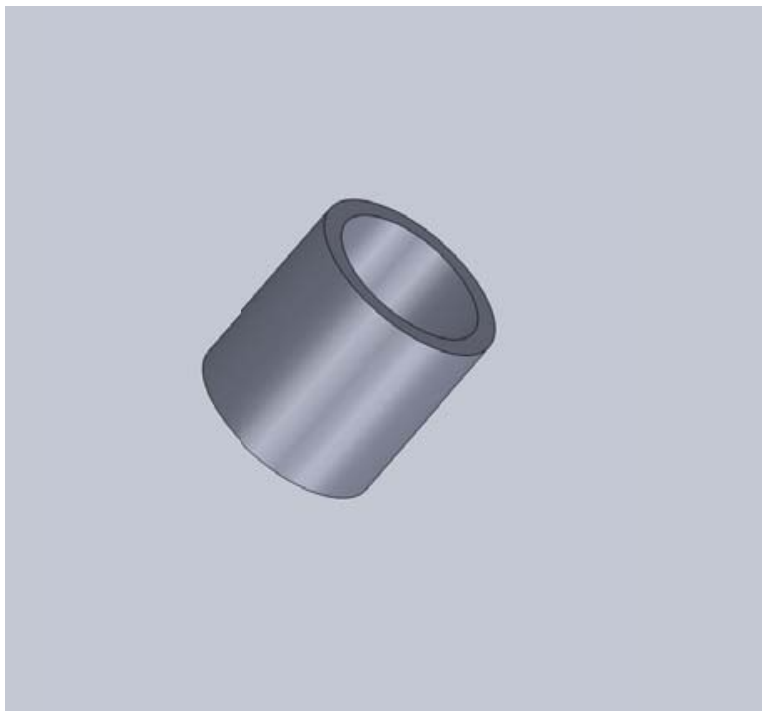

Figure 5.11: Precise cylinder geometry.

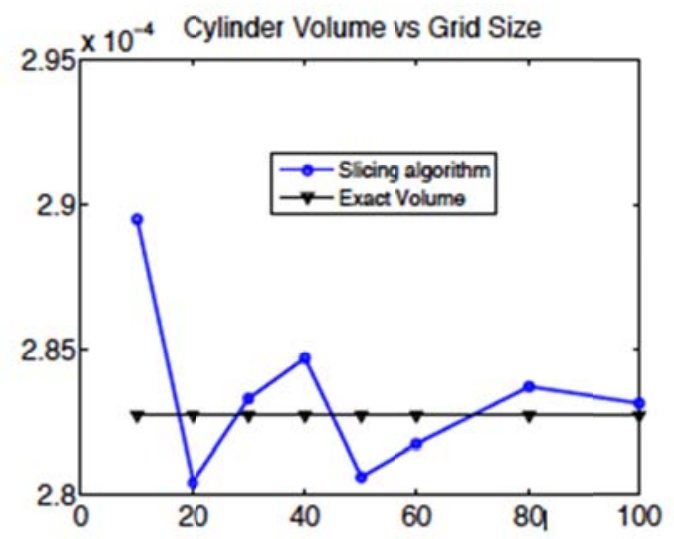

Figure 5.12: Precise cylinder volume vs grid size. 


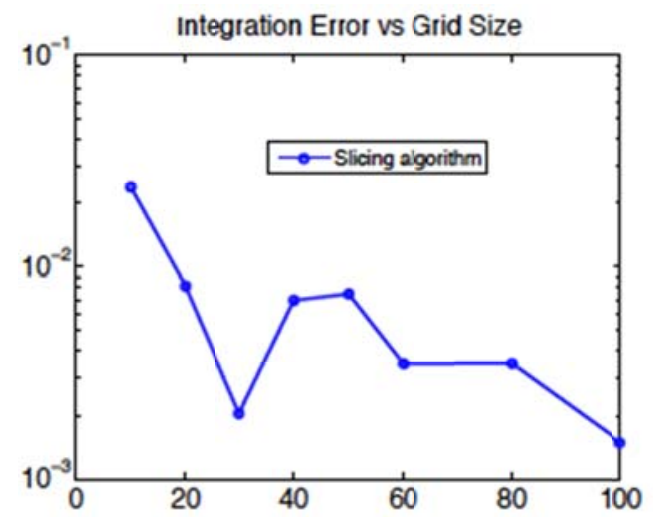

Figure 5.13: Integration errors vs grid size.

slices remark the fluctuation and the tendency of the algorithm to converge and pose to a reliable value at large number of slices. From $60 \times 60 \times 60$ to $80 \times 80 \times 80$ slices; the algorithm keep a conservative and non fluctuant result until a final convergence when using 100x100 slices grid cells.

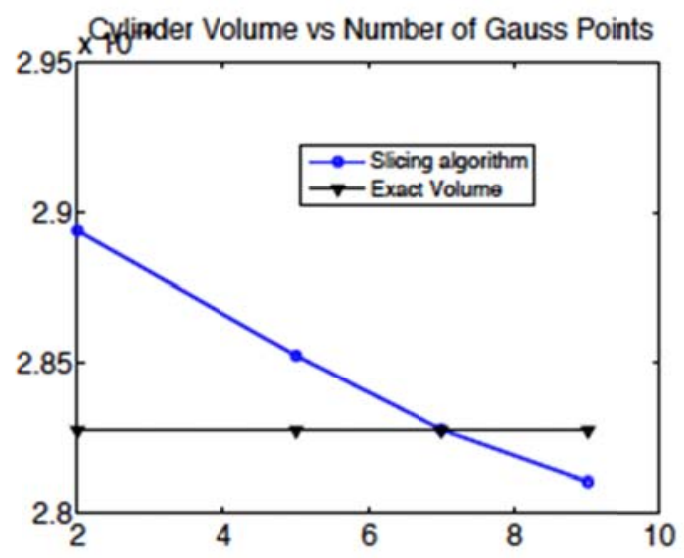

Figure 5.14: Precise cylinder volume vs gauss points.

Figure 5.14 For this perfect geometry as well as the bracket geometry mentioned before the use of more than 5 number of gauss integration points can o er a better convergence and stability numerical approximation toward the exact value; in this particular case 7 gauss points were necessary to get an acceptable result.

Figure 5.15 at 7 gauss points the integration error is reduced at its minimum accountable; 


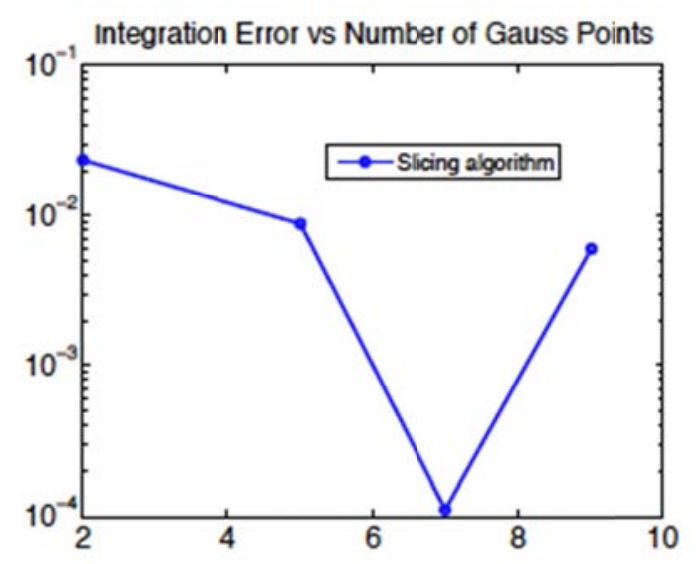

Figure 5.15: Integration error vs number of gauss points.

having the best value when using a 20x20x20 slicing matrix. The experimental data results obtained for this perfect geometry model (cylinder) can suggest that a balance of number of integration points and the right choose of the number of slicing layers in 2D are necessary to get a better numerical approximation and convergence of the integrand.

For cylinder model with imprecise geometry:

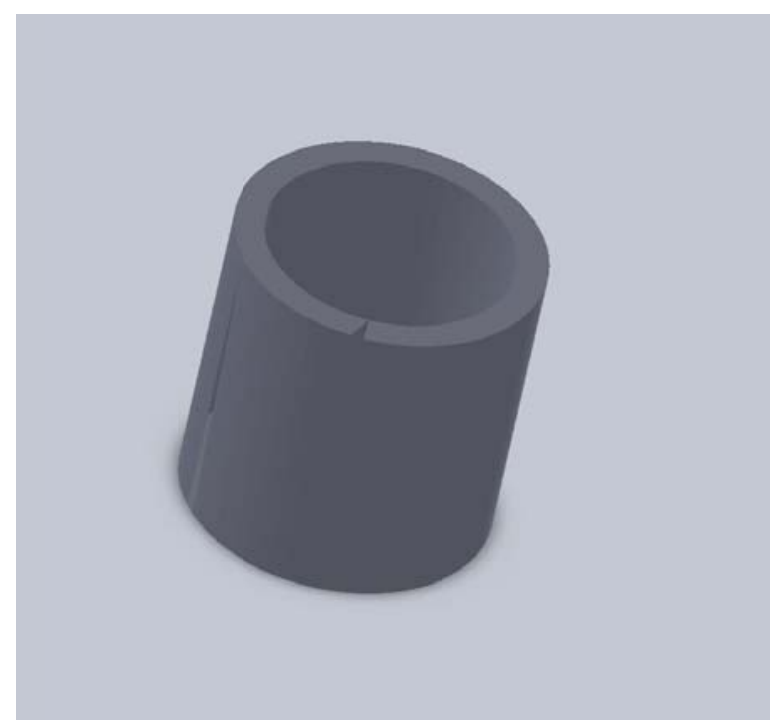

Figure 5.16: Imprecise cylinder geometry.

The figure 5.16 represent our model for an imprecise cylinder geometry.

Figure 5.17 Similar to the first plot for perfect geometry the slicing algorithm for im- 


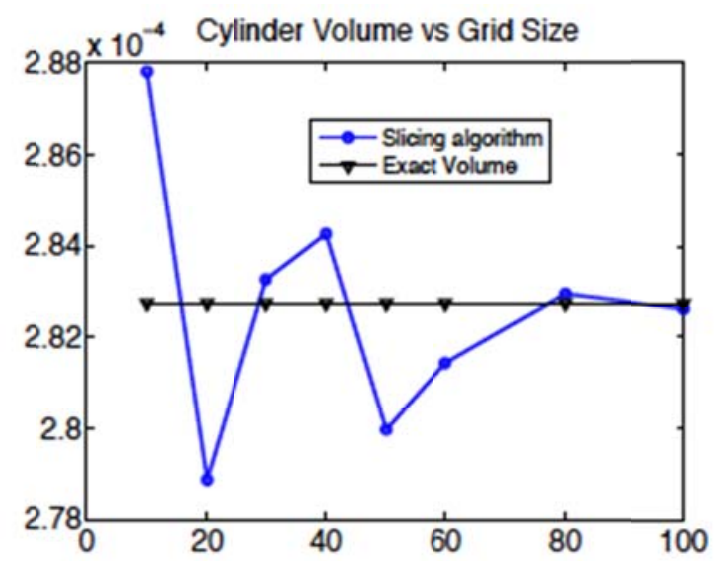

Figure 5.17: Imprecise cylinder volume vs grid size.

perfect geometry converge rapidly to the exact volume value. It shows oscillating values computed at lower slicing grids but with a significative convergence toward the exact volume value. The plot shows a consistency in the experimental computed volume value with the real value from $80 \times 80$ grids up to $100 \times 100$ grids; suggesting that 100 slicing grids with 3 gauss allocation points could be considered a perfect convergence solution.

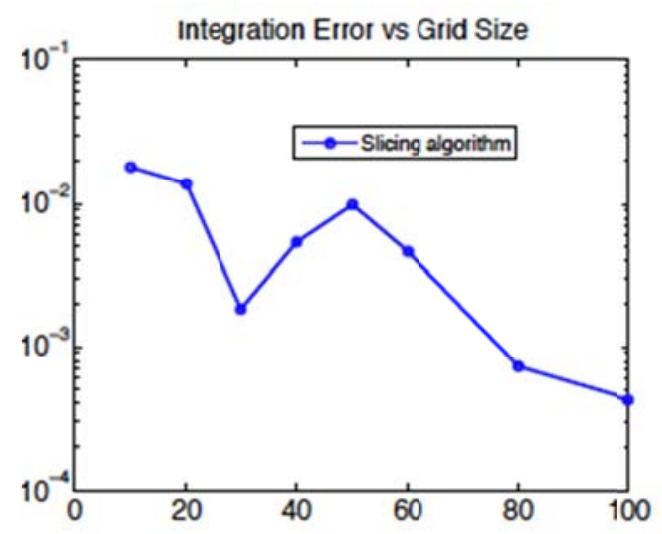

Figure 5.18: Integration errors vs grid size.

Figure 5.18 The integration errors consistently vary and have a decreasing convergence toward a minimum error solution at 100x100 slices grid. At 30x30x30 slices can be observed with a momentary rough decline of error and then return to the slope tract convergence. 


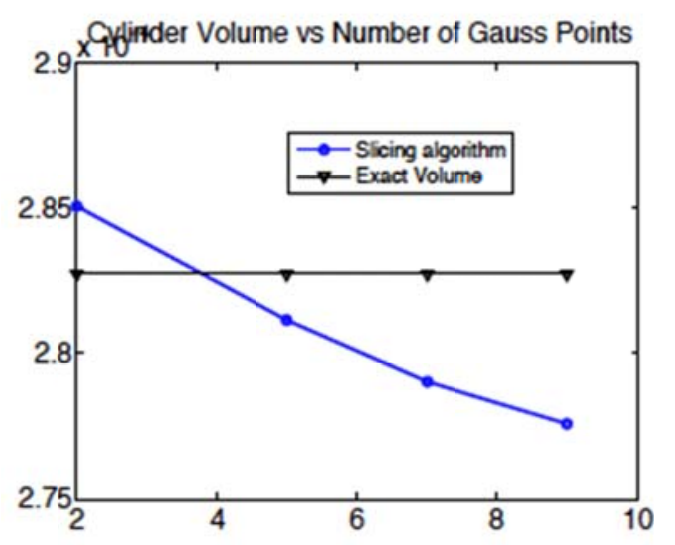

Figure 5.19: Imprecise cylinder volume vs gauss points.

Figure 5.19 At 4 allocated integration points the only acceptable convergence solution is matched with the real value. The lack of convergence as an increase in the gauss points is due to the poor density of slices. A 20x20-slicing grid is not su cient to have a good result.

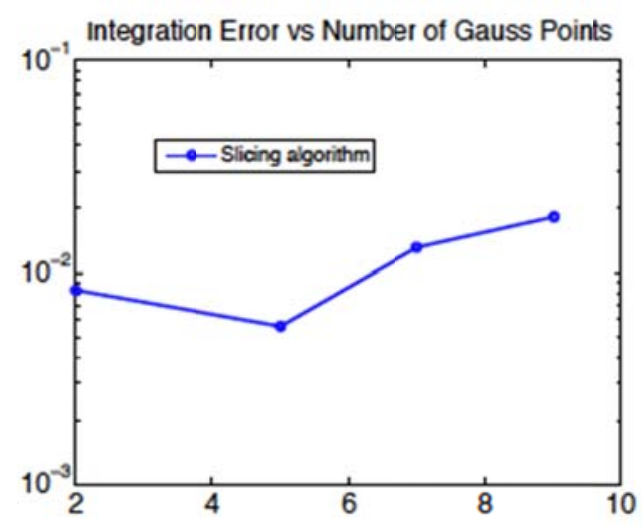

Figure 5.20: Integration error vs number of gauss points.

Figure 5.20 Integration error has poor variation when increasing the number of gauss integration points. Some light variation can be noticed when using 5 gauss points and placing only a 20x20 slice grids for this imperfect model, which reduces the accuracy of the results. However, the integration has stable computational errors.

\subsubsection{Numerical experiment 3: 3D volume computation in a Spur Gear}


The spur gear model that will be used for the next experiment was imported directly from an educational website called GrabCAD. The purpose is to try to use our non-designed model and apply the slices 3D algorithm integration method to a model designed by others using their own modeling package. The treatment of this model and the plots generated will have the same pattern than the other 2 models proposed. The exact volume of this model was obtained from SOLIDWORKS and uses the same tool command for volume evaluation. The Figure 5.21 is the model for a precise spur gear model. The following plots contain the same parameter coordinates as the other plots already shown.

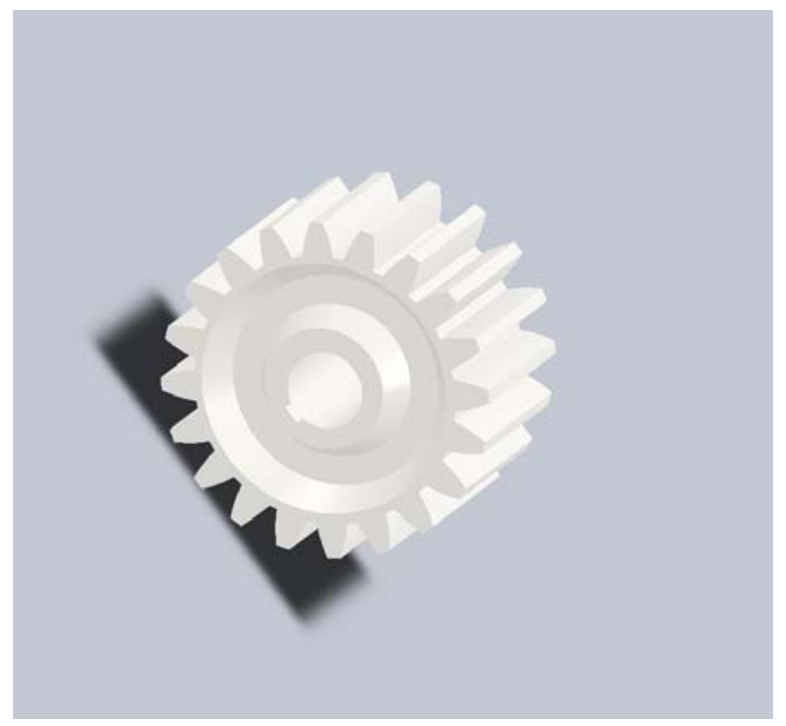

Figure 5.21: Precise geometry.

The Figure 5.21 represent our model for a precise spur gear geometry.

Figure 5.22The plot shows an oscillating volume value using di erent grid densities. The oscillation has a trend to converge to the exact value after using 60x60 slices. Incrementing the density of slices improves the convergence between 80x80 and 100x100 slices.

Figure 5.23 Integration errors decrease consistently when we increase the grid 3D densities. The lower integration error is found at 100x100x100 grid density. 


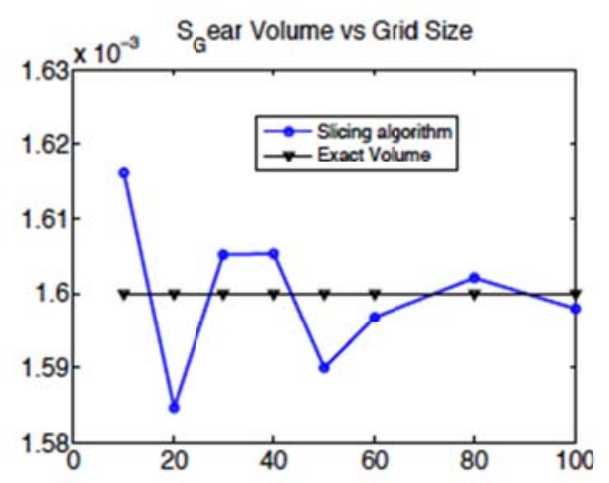

Figure 5.22: Spurge volume vs grid size.

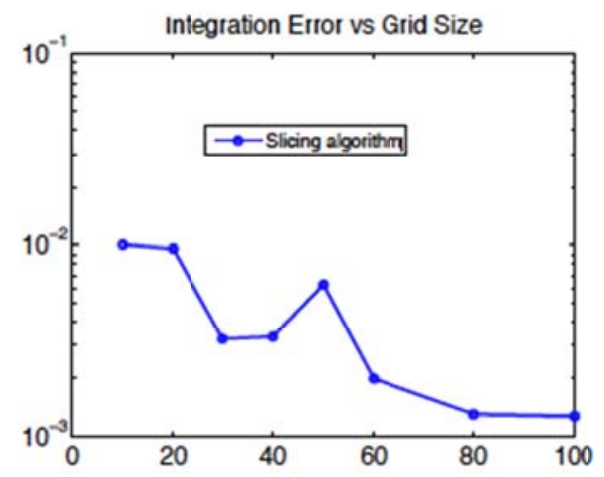

Figure 5.23: Integration errors vs grid size.

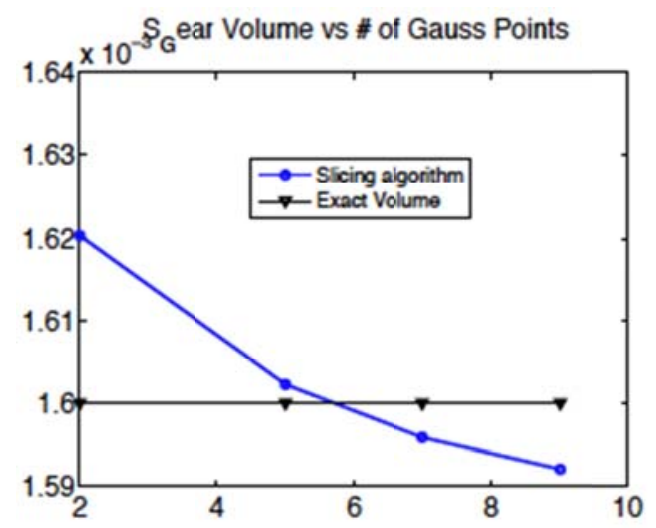

Figure 5.24: Spurge volume vs gauss points. 
Figure 5.24 We have obtained a fast volume value convergence using 5 gauss points which represent the exact volume value. Increasing the numbers of gauss points does not improve the convergence but keeps a relative di erence volume error related with the exact value of (1.0 $)^{-} 3$ units which is a good approximation volume error.

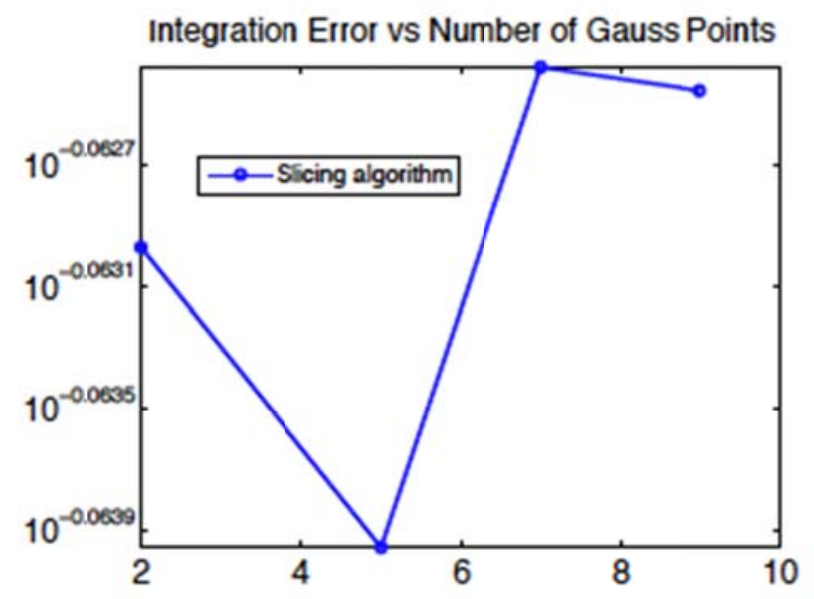

Figure 5.25: Integration error vs number of gauss points.

Figure 5.25 Even if the graphic apparently shows large changes between two successive points, the large di erence between both extremes is $(1.2)^{-} 3$ which is a relatively good conservative error in terms of volume di erence. For this experiment, the increment of the number of gauss points does not notably improve the integration errors.

The Figure 5.26 represents our model for an imprecise spur gear geometry. Figure 5.27A perfect approximation and convergence to the exact volume is found at 80x80 slices, but it is possible to notice that with a lower number of slicing (30x30 and $40 \times 40$ ) the convergence to the exact volume is fast and accurate. The slicing $100 \times 100$ also o ers a good solution.

Figure 5.28 Integration errors are very alike and are located in a similar range of approximation. The minimum error is found when $80 x 80$-slicing grids are used. The integration errors for this experiment, using an imprecise geometry, do not vary largely using di erent grid densities

Figure 5.29 This experiment shows a lack of stability when increasing the number of 


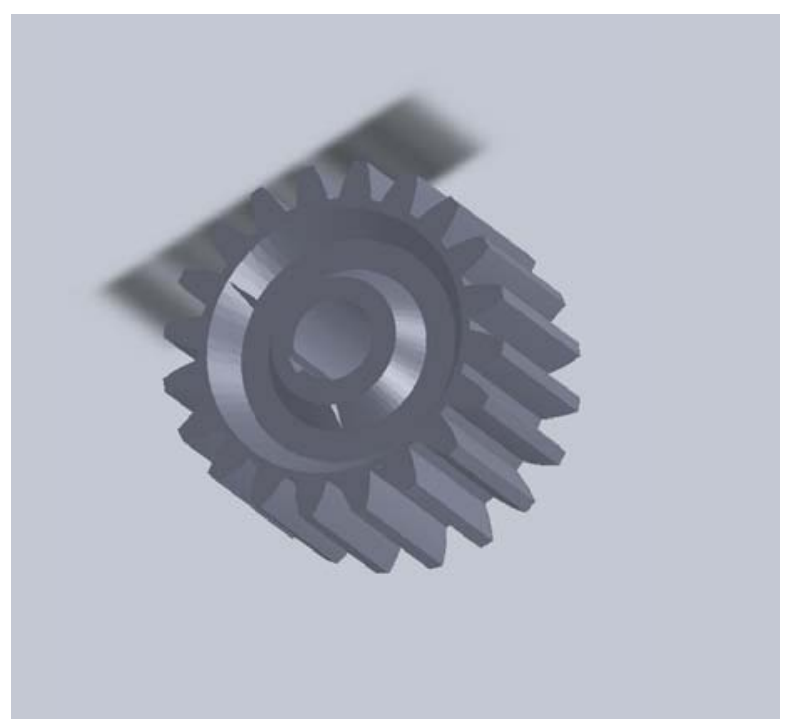

Figure 5.26: Imprecise geometry.

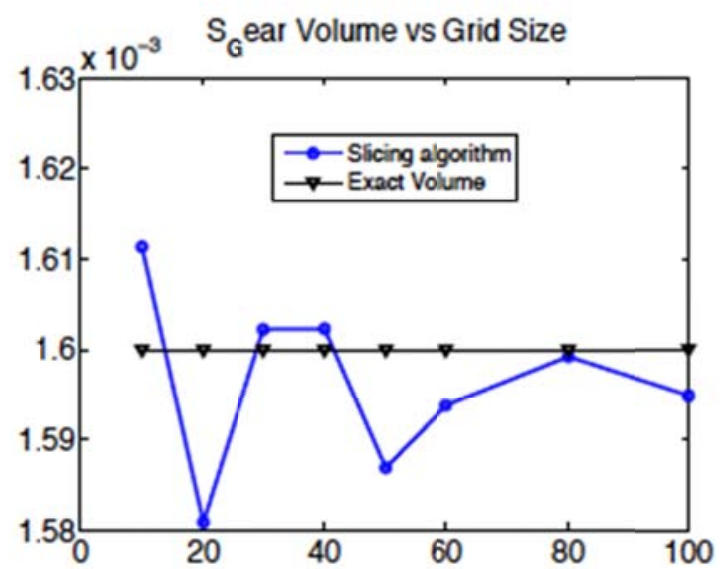

Figure 5.27: Spurge volume vs grid size.

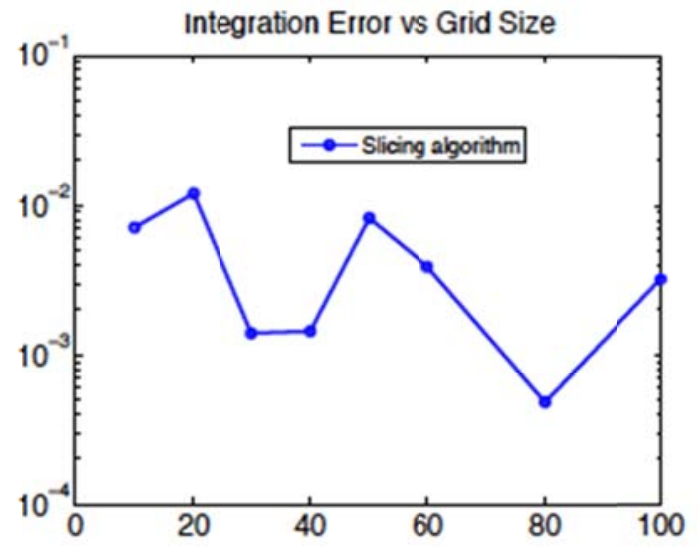

Figure 5.28: Integration errors vs grid size. 


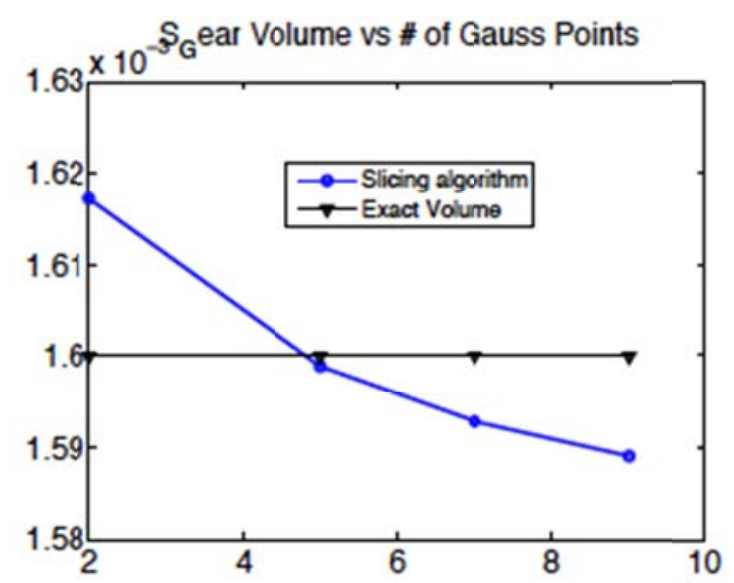

Figure 5.29: Spurge volume vs gauss points.

gauss points. At 20x20 slicing the only value with an exact solution is when 5 gauss points are used. The volume value has a conservative oscillation of less than (1.0) 3 cubic meters.

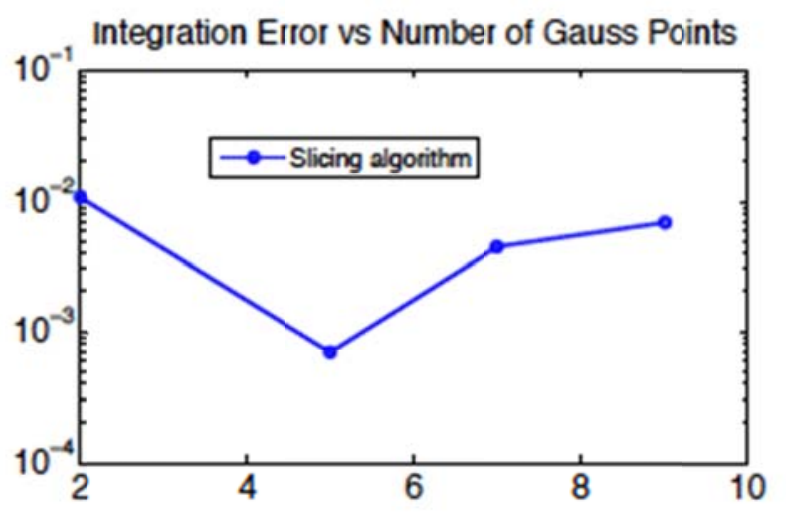

Figure 5.30: Integration error vs number of gauss points.

Figure 5.30 Integration errors using di erent gauss points for the imprecise model do not show much di erence from the precise model. Indeed, the change of errors is very conservative. The lower error is produced when using 5 gauss points.

\subsubsection{Preliminary volume evaluation conclusions}

For this first evaluation of the proposed 3D slices method, we have evaluated the volume of three di erent solid models using either a perfect geometry or imperfect geometry conformation. Evaluation and plotting of four di erent numerical experiments 
were done as:

- Computed volume vs grid size.

- Integration error vs grid size.

- Computed volume vs number of Gauss points.

- Integration error vs number of Gauss points.

The proposed 3D slices method converges satisfactorily when we increase the numbers of gauss points and the number of grid slices allocated. We can guarantee a good convergence either for a perfect or imperfect model. However, in some cases the convergence is found with a lesser number of integration points and number of grid slices. For completeness of the numerical experimentation it is preferred to set the parameters with larger numbers as we have used during the experimentation shown previously.

\subsection{Numerical experiment 4}

The next two validation experiments will be applied for 3D natural vibration analysis using the meshfree solution structure method. The experiments processed before will be compared with both results using models with perfect geometry and imperfect geometry, due to internal gaps. The [18] describes the accuracy and convergence of the computed natural frequencies implicating the exact treatment of the prescribed boundaries' conditions that are enforced using approximate distance functions that vanish at the boundaries of the geometrical model. The SSM will be applied for the next two 3D models and will be the objective of this experiment to evaluate the natural frequencies for the imperfect model using the algorithms and codes written to analyze the imperfect model without repairing and compare the results with the same geometries but without gaps and geometrical imperfections (perfect geometry). No commercial or well-known mechanical analysis software is able to run or obtain acceptable solutions when the geometry is damaged or imperfect. Actual commercial software repairs the 
models forcing a change to the original geometry. Most of them just stop running. In Figure5.31 represents a damaged bracket model using the SOLIDWORKS analysis package. In Figure5.32; ANSYS released negative comments related to the damaged bracket under study. Using either the analysis package o ered by SOLIDWORKS or the analysis package from ANSYS, the results are the same. For a geometric damaged model the analysis solver tool just stops running. SOLIDWORKS at least allows one to see the geometric damaged areas. ANSYS, instead, released a text editor. In Figure5.33ANSYS edits the damaged bracket geometry chart and the Figure5.34 shows the same bracket but without geometric damages. In general, the well-known finite element analysis constitutes a usual numerical method to analyze dynamic engineering problems. However, the use of spatial grids conforming the shape of the geometric model can represent a di cult trend to evaluate engineering physical time dependence problems forcing simplification or repair of the geometric model and a change to the real physical geometry of the object.

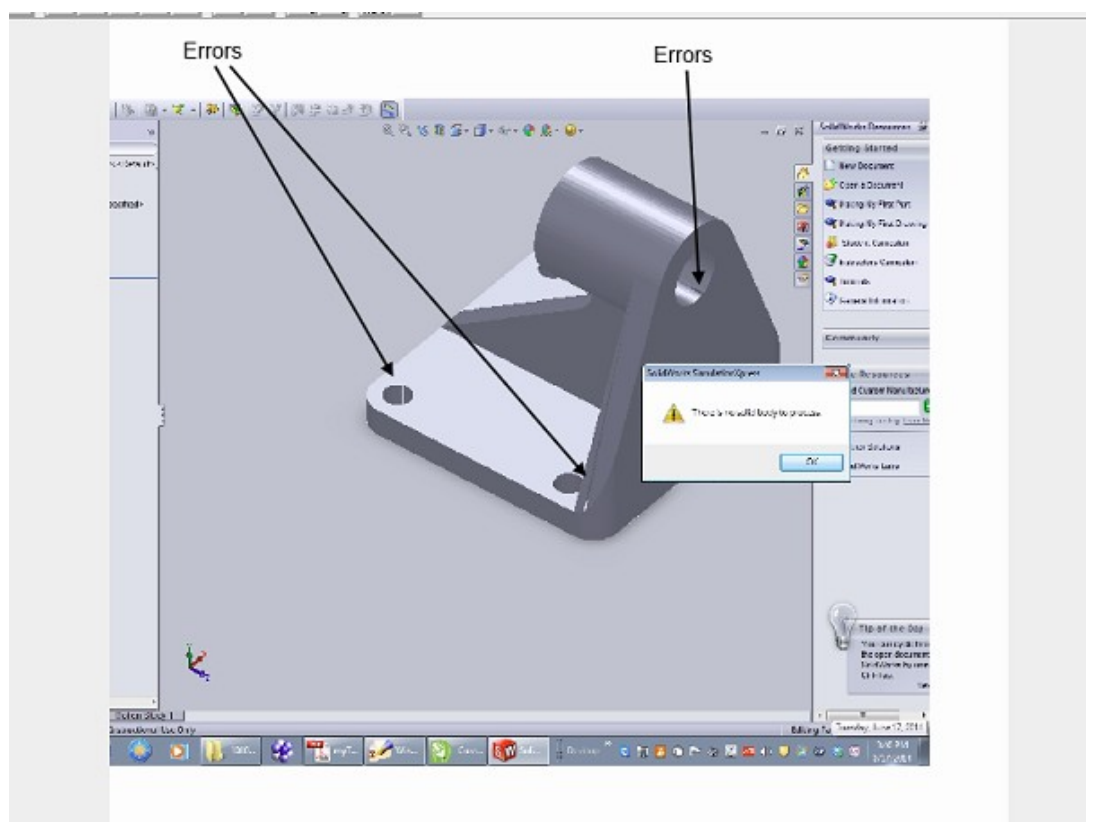

Figure 5.31: SolidWork Editor for imprecise bracket model.

For natural vibration experiments we will use a solution structure that satisfy kinematic 


\begin{tabular}{|c|c|c|c|}
\hline & A & B & c \\
\hline 1 & Type & Text & Association \\
\hline 2 & Error! & $\begin{array}{l}\text { Update failed for the Model cell in Static Structural (ANSYS). Eror updating cell Model in system Static Structural (ANSYS). } \\
\text { view the messages in the Mechanical editor for more detals. }\end{array}$ & \\
\hline 3 & Error! & $\begin{array}{l}\text { Update of the Model cell in Static Structural (ANSYS) falled: Error updating cell Model in system Static Structural (ANSYS). View } \\
\text { the messages in the Mechanical editor for more detals. }\end{array}$ & \\
\hline 4 & Errorl & Error updating cell Model in system Static Structural (ANSYS). View the messages in the Mechanical editor for more detals. & \\
\hline 5 & Errorl & Error updating cel Model in system Static Stuctural (ANSYS). View the messages in the Mechanical edtor for more details. & \\
\hline 6 & Error! & Error updating cel Model in system Static Structural (ANSYS). View the messages in the Mechanical editor for more details. & \\
\hline 7 & Informational & The Model cel in Static Structural (ANSYS) requires user input before it can be updated. & \\
\hline 8 & Error! & $\begin{array}{l}\text { The following cells need attention before proceeding: } \\
\text { Geometry }\end{array}$ & \\
\hline 9 & Error! & $\begin{array}{l}\text { The following cells need attention before proceeding: } \\
\text { Geometry }\end{array}$ & \\
\hline 10 & Warning! & 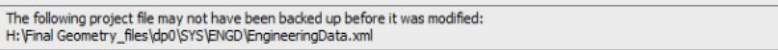 & \\
\hline 11 & Errorl & $\begin{array}{l}\text { The following cells need attention before proceeding: } \\
\text { Geometry }\end{array}$ & \\
\hline 12 & Error! & $\begin{array}{l}\text { The following cells need attention before proceeding: } \\
\text { Geometry }\end{array}$ & \\
\hline 13 & Eror! & $\begin{array}{l}\text { The following cells need attention before proceeding: } \\
\text { Geometry }\end{array}$ & \\
\hline 14 & Errorl & $\begin{array}{l}\text { The following cells need attention before proceeding: } \\
\text { Geometry }\end{array}$ & \\
\hline 15 & Error! & $\begin{array}{l}\text { The following cells need attention before proceeding: } \\
\text { Geometry }\end{array}$ & \\
\hline 16 & Error! & $\begin{array}{l}\text { The following cells need attention before proceeding: } \\
\text { Geometry }\end{array}$ & \\
\hline 17 & Error! & $\begin{array}{l}\text { The following cells need attention before proceeding: } \\
\text { Geometry }\end{array}$ & \\
\hline \multicolumn{4}{|c|}{ rogress } \\
\hline & & $A$ & \\
\hline
\end{tabular}

Figure 5.32: Ansys text editor for damage geometric model.

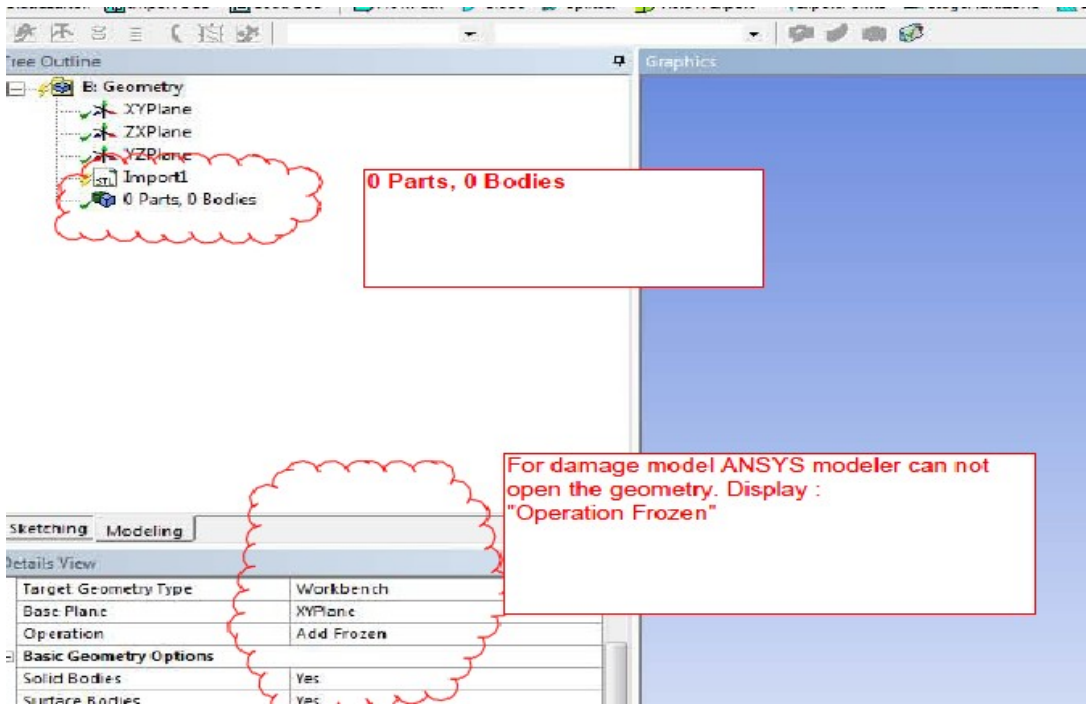

Figure 5.33: Imprecise bracket model. 


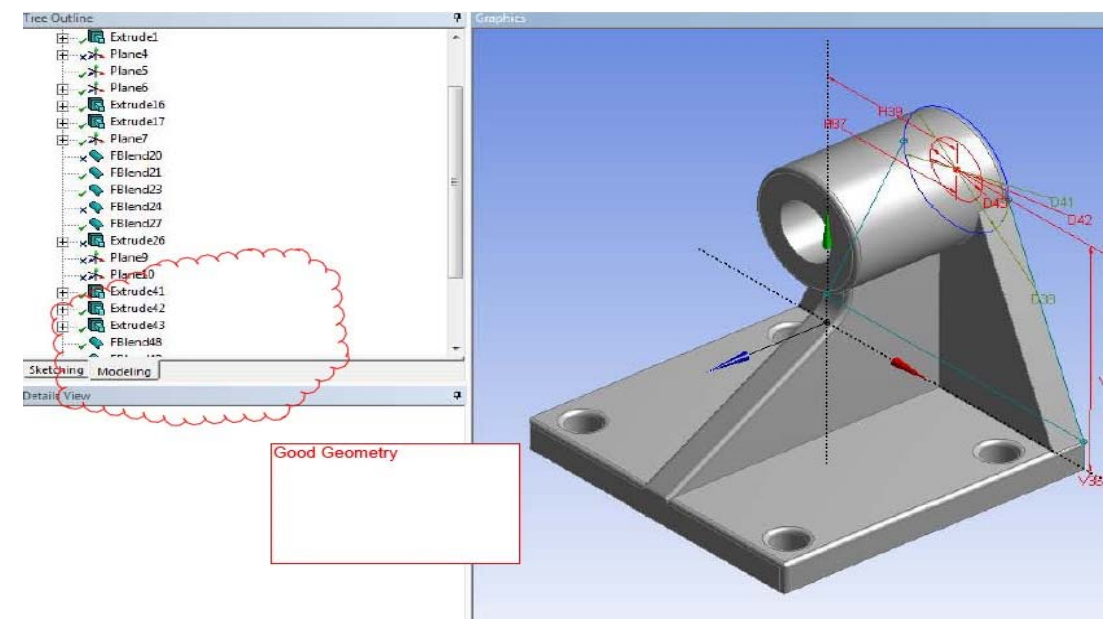

Figure 5.34: Precise bracket model.

(essential type) boundary conditions that are formulated as zero displacement along the coordinate axis.

$$
\begin{aligned}
& \left.u_{x}\right|_{\partial \Omega}=0 \\
& \left.u_{y}\right|_{\partial \Omega}=0 \\
& \left.u_{z}\right|_{\partial \Omega}=0
\end{aligned}
$$

Since not all displacement components can be set to zero at the same geometric boundaries, kinematic boundary conditions include both fixed and sliding boundaries. To satisfy these boundary conditions we will use a vector solution structure.

$$
\mathbf{u}_{i}=\omega_{i} \phi_{i}=\sum_{j=1}^{n} C_{j}{ }^{i}\left(\omega_{i} \chi_{j}\right), i=x, j, z
$$

Natural vibration in mechanical structures is governed by the balance between the work 
done by the inertial forces and the potential energy of the elastic deformation.

$$
\int_{\Omega} \epsilon^{T} \boldsymbol{\sigma} d \Omega=-\int_{\Omega} \mathbf{u}^{T} \rho \ddot{\mathbf{u}} d \Omega,
$$

The energy balance equation formulated above can be expressed using a generalize eigenvalue problem as:

$$
[\mathbf{K}][\boldsymbol{\varphi}]=\lambda^{2}[\mathbf{M}][\boldsymbol{\varphi}],
$$

The formula described above denotes $\mathrm{K}$ as the sti ness matrix and $\mathrm{M}$ as mass matrix; $\boldsymbol{\varphi}$ is an amplitude of the displacement and $\lambda$ is a natural frequency. As mentioned before the amplitude $\boldsymbol{\varphi}$ can be expressed by the following solution structure:

$$
\phi=\sum_{i=1}^{n} C_{i}\left(\omega_{x}, \omega_{y}, \omega_{z}\right)^{T} \chi_{i}
$$

Choosing $\boldsymbol{\eta}$ as basis function satisfying essential boundary conditions (Dirichlet) the sti ness and the mass matrices can be expressed as:

$$
\begin{gathered}
K_{i j}=\int_{\Omega} \mathrm{B}^{T}\left[\boldsymbol{\eta}_{i}\right] \mathrm{DB}\left[\boldsymbol{\eta}_{j}\right] d \Omega, \\
M_{i j}=\int_{\Omega} \boldsymbol{\eta}_{i} \rho \boldsymbol{\eta}_{j} d \Omega,
\end{gathered}
$$

Where $\boldsymbol{B}$ denotes the matrix of derivatives, also known as the strain-displacement matrix, so that $\boldsymbol{\epsilon}=\boldsymbol{B}[\boldsymbol{\varphi}]$, and $\boldsymbol{D}$ represents the stress-strain matrix so that $\sigma=\boldsymbol{D} \boldsymbol{\epsilon}$. The architecture of the solution designed for this specific example will use tensor product B-spline basis functions. Matrices in this case are sparse meaning that most of the elements are zero. We will use a Lanczos method to solve a generalized eigenvalue problem for sparse Hermitian matrices combined with an inverse spectrum transformation. We 
will consider the following eigenvalue problem:

$$
[\mathbf{K}]^{-1}[\mathbf{M}][\boldsymbol{\varphi}]=\theta^{2}[\boldsymbol{\varphi}]
$$

In this section, the object of the study will be two di erent kinds of models. First, we will use a bracket already designed and used to evaluate volume using a perfect model and an imperfect model. Second, we will use a spur gear. This model was downloaded from the Internet and is called "Track Parts," an external supplier of mechanical models. It allows us to demonstrate and validate the algorithm application for any kind of model. The experiments will determine the convergence of the algorithm applied to a perfect model and the relative imperfect model. In this case, we will apply SSM for natural vibration including the algorithm to analyze the imperfect model without repairing the model and its boundaries.

\subsubsection{Numerical experiment 5}

In this section, we will propose an application of the meshfree Solution Structure Method for the mechanical analysis of natural vibration problems in 3D models with geometrical imperfections. The study of natural vibration in mechanical models represents a very strict guideline design required in major mechanical analysis problems that must guarantee the good performance of a mechanical structure when applied to dynamics loads. Missile and propulsion rockets are classic examples for which this kind of analysis is required. Natural vibration analysis is required for a time-varying loading condition in order to determine the structure's system response. Experimentation samples include: 1-bracket, and 2-spider bracket Both represent classical mechanical structural parts commonly used in aeronautical application. The oscillating dynamics loads in these kinds of models require an accurate mechanical analysis to obtain the proper behavior of the elements under dynamics stresses. Finite Element Analysis (FEA) has been the classic numerical method used to analyze this kind of problem. However, the presence of ge- 
ometrical model imperfections like gaps, as well as very small geometrical details that conform the boundaries, force the designer to change the mechanical and geometrical structure to avoid excessive time analysis or eventual repair. The use of spatial grids that conform the model can degenerate the real cause of study forcing the design of another geometrical model that is not desired. The introduction of a Meshfree Solution Structure Method using distance field to the boundaries can guarantee an accurate approach solution analysis to the geometry without the need to repair or change the desired model under analysis. It is due to study and validation in this work that the algorithm proposed could supply and derive an approximate solution in cases when the geometrical model has imperfections. The cases proposed previously will be analyzed for either perfect geometric model or imperfect geometric models. The investigation process defines a research procedure to obtain natural vibration modes for the samples mentioned. In order to obtain the right parameters to compare the di erent natural frequencies for both types of models using precise and imprecise geometries, the natural frequencies obtained from the SOLIDWORKS simulation was taken using the precise models. The Solution Structure Method (SSM) proposed slicing parallel layers for the allocation gauss integration points to generate a Cartesian 3D grid with accountable small grid sizes. Same grid size is used as a mesh size parameter h; which can be used as input in SOLIDWORKS as a meshing parameter definition. This procedure is used to keep the same meshing domain and to be able to compare the solution obtained by SSM and FEA. The SOLIDWORKS mesh size h can be set manually as input. The same mesh size parameter used in the precise model will be used to compare with the imprecise model since SOLIDWORKS cannot process imprecise models. Plots will be released using MATLAB tools and the modeler will release 10 natural frequency modes through the SSM proposed method. MATLAB plots will show four di erent mode vibrations, modes $1,2,3,10$. Each one will show the change of the natural frequency in 
$\mathrm{Hz}$ with respect to the change of the mesh size $\mathrm{h}$ in meters. Experiments will be done for bracket and spur gear solid models for precise models and imprecise models.

The following plots represents natural vibration modes experiments for precise bracket models

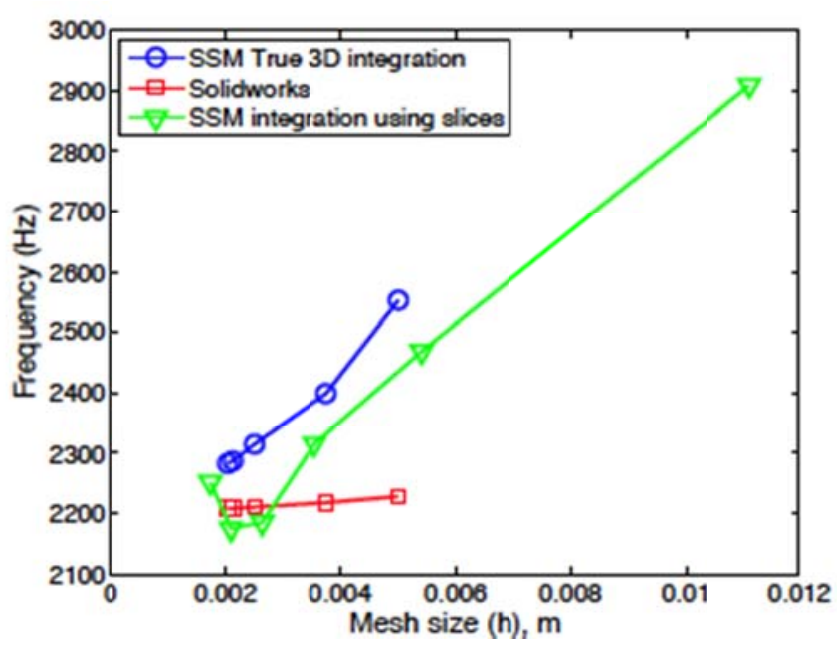

Figure 5.35: No damage bracket natural vibration Mode 1.

Values for frequencies in Figure5.35show conversion to the SOLIDWORKS values when comparing at lower frequency like $2200 \mathrm{~Hz}$ while using the proposed slices integration method in the range of meshing size between $0.002 \mathrm{~m}$ and $0.003 \mathrm{~m}$. The true 3D integration method converges as well to the SOLIDWORKS values. For these kinds of plots, and as a manner to define good results, when we reduce the mesh size we find convergence among the three integration methods.

for the second mode of natural vibration, using a precise bracket model, the plot shows a similarity of convergence among the three methods under study when the mesh size is decreased. We can notice that for this mode 2 of vibration, the range of convergence increases using mesh sizes between 0.002 and $0.004 \mathrm{~m}$.

At Mode 3 Figure5.37convergence of the three methods is found when decreasing the mesh size. The proposed slices integration method converges very much the way it does in SOLIDWORKS between the mesh sizes of 0.002 and $0.004 \mathrm{~m}$. 


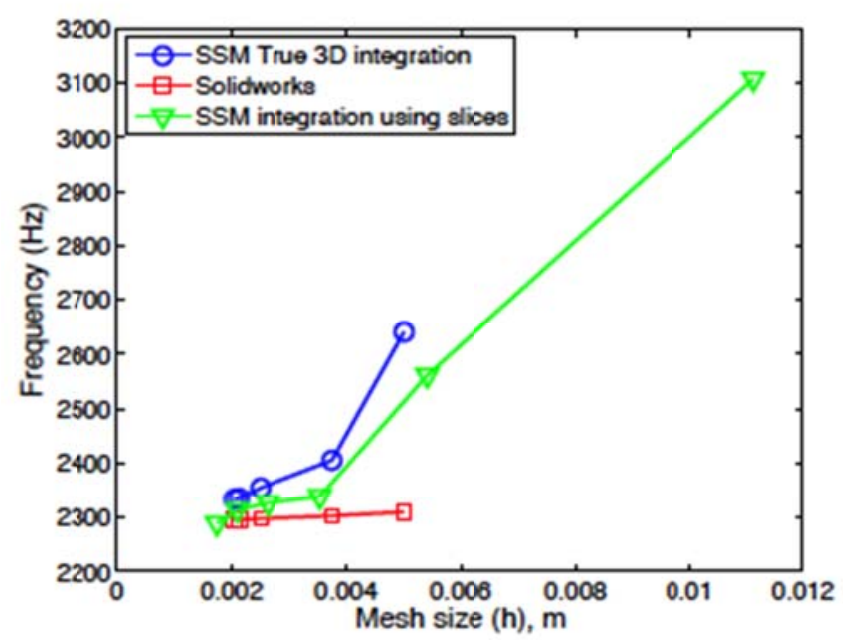

Figure 5.36: No damage bracket natural vibration mode 2.

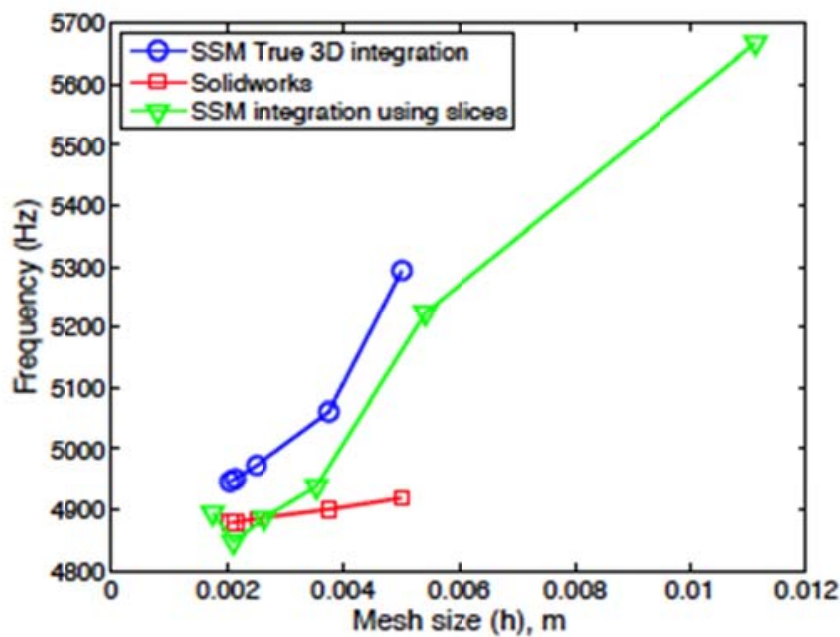

Figure 5.37: No Damage Bracket Natural Vibration Mode 3. 


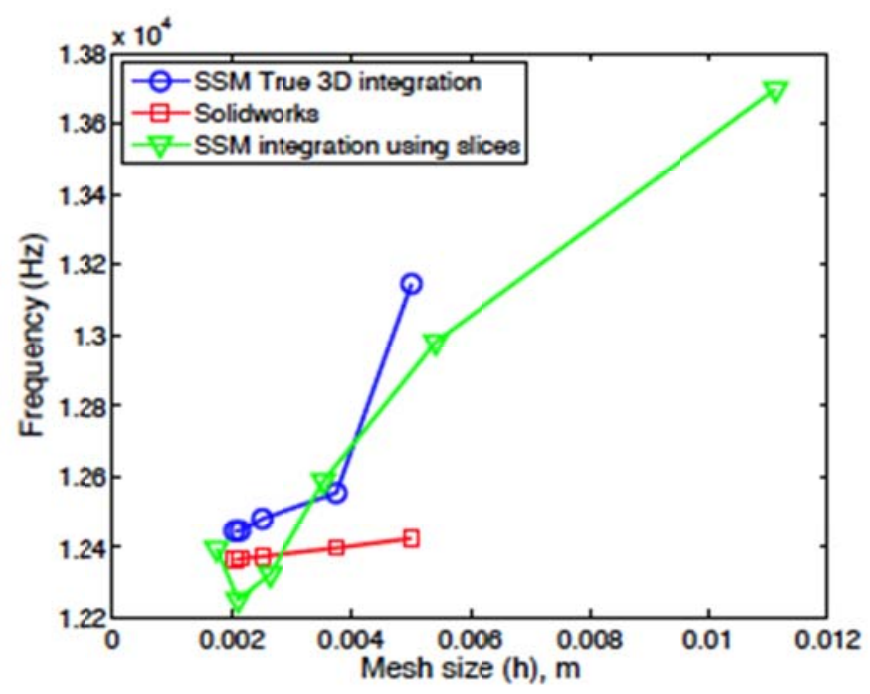

Figure 5.38: No Damage Bracket Vibration Mode 10.

At this mode vibration 10 Figure5.38 shows a full convergence again of the three integration methods used. We can observe at the plot that between $0.002 \mathrm{~m}$ and $0.004 \mathrm{~m}$ mesh sizes, we obtain similarities among the natural frequencies computed.

The following 4 plots represents the convergence of the natural vibration modes experiments for a precise bracket model when compare with the solid work values

The Figure5.39 shows the convergence of the natural frequency Mode 1 to the Solid Work value when increasing grid refining

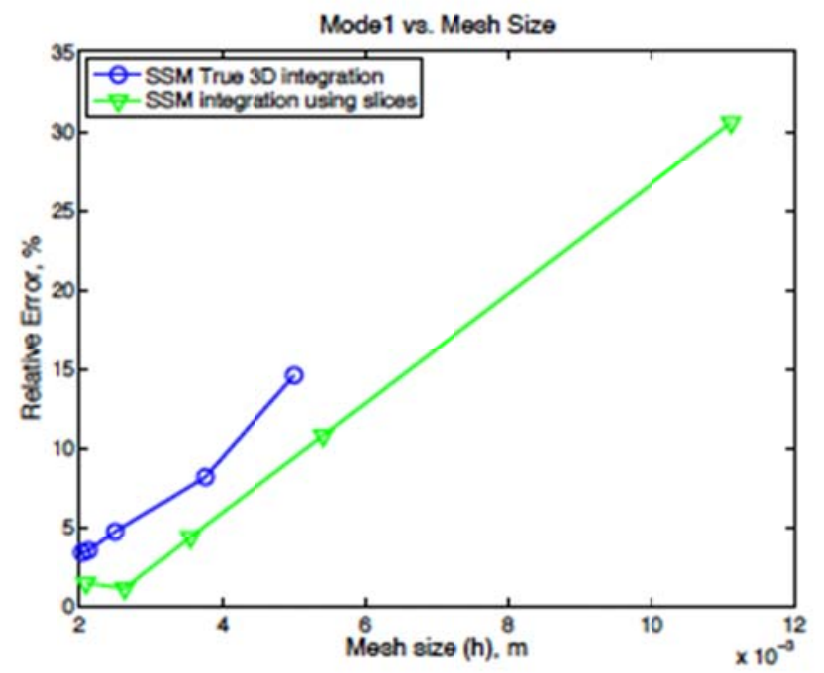

Figure 5.39: No Damage Bracket Vibration Convergence Mode 1. 
The Figure5.40 shows the convergence of the natural frequency Mode 2 to the Solid Work value when increasing grid refining

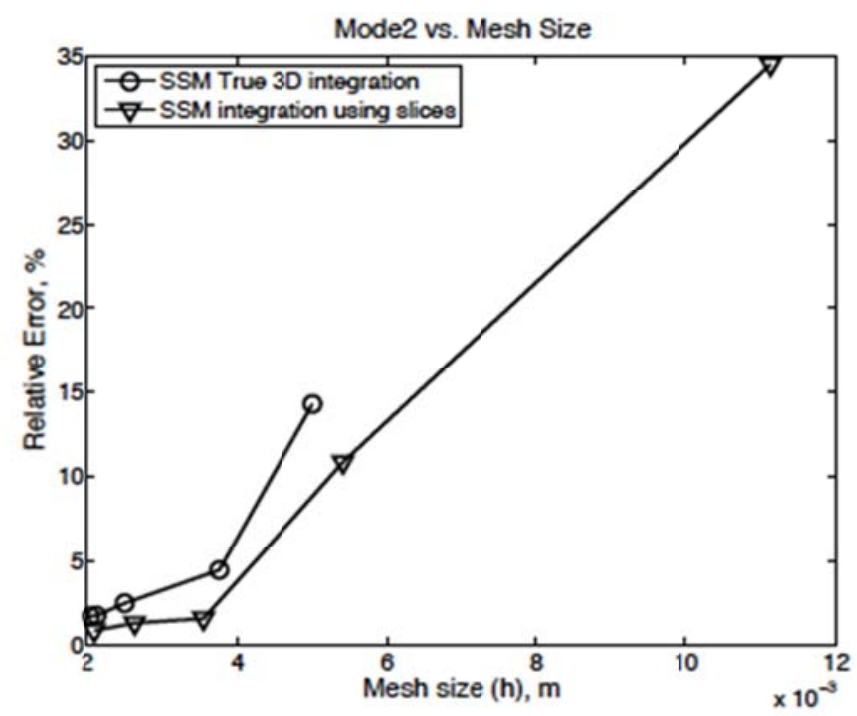

Figure 5.40: No Damage Bracket Vibration Convergence Mode 2.

The Figure5.41 shows the convergence of the natural frequency Mode 3 to the Solid Work value when increasing grid refining

The Figure5.42 shows the convergence of the natural frequency Mode 10 to the Solid Work value when increasing grid refining

The following plots represents natural vibration modes experiments for an imprecise bracket model; the Figure5.43 shows a damage geometry. . At the internal main hub it is possible to see the lack of a piece of surface.

For this first Mode Figure5.44 of natural vibration, for an imprecise bracket model, in the true 3D integration method, the proposed slicing method and the SOLIDWORKS results follow the same path for a full convergence. Using lower mesh sizes accelerate the convergence.

For the second mode Figure5.45 of natural vibration, using an imprecise bracket model, the plot shows a similitude of convergence among the three methods under study, and when we decrease the mesh size, we can see that for this mode 2 of vibration, the range of 


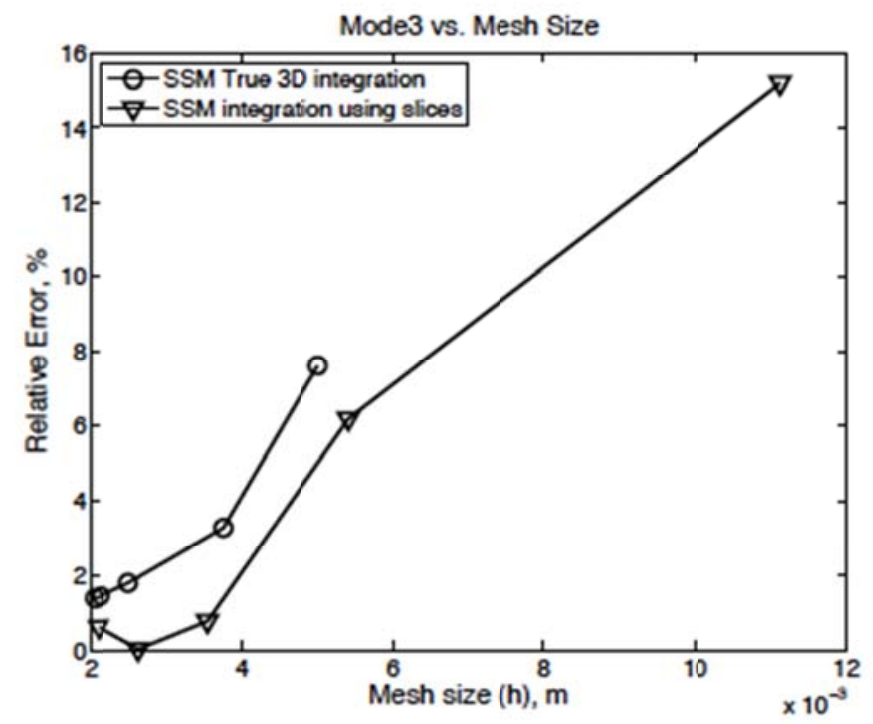

Figure 5.41: No Damage Bracket Vibration Convergence Mode 3.

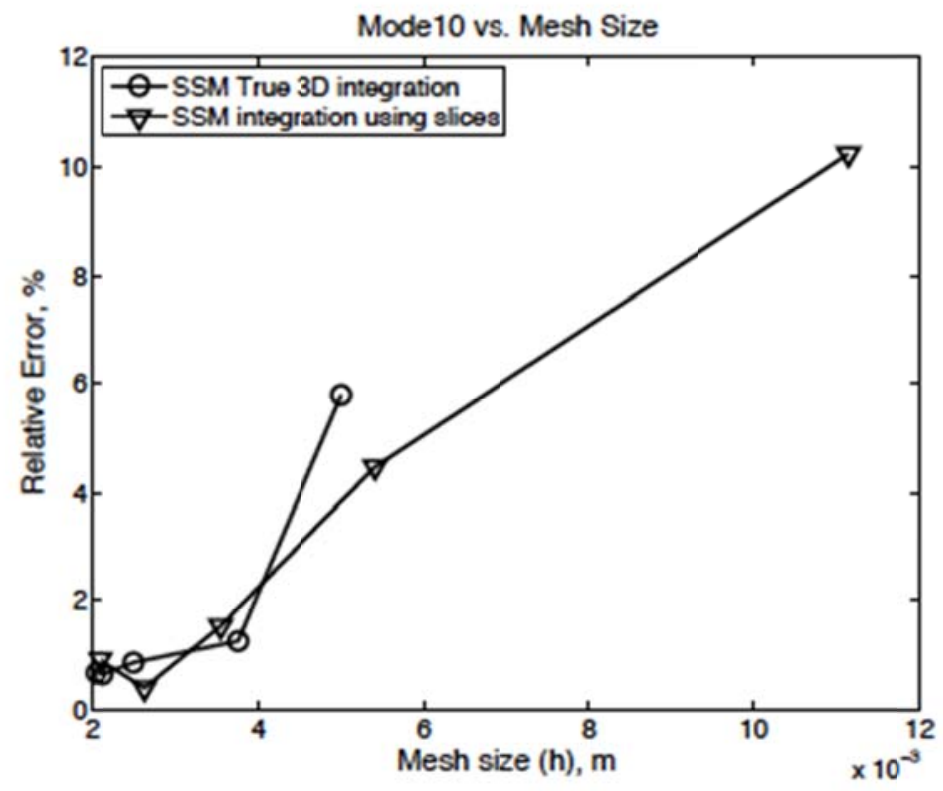

Figure 5.42: No Damage Bracket Vibration Convergence Mode 10. 


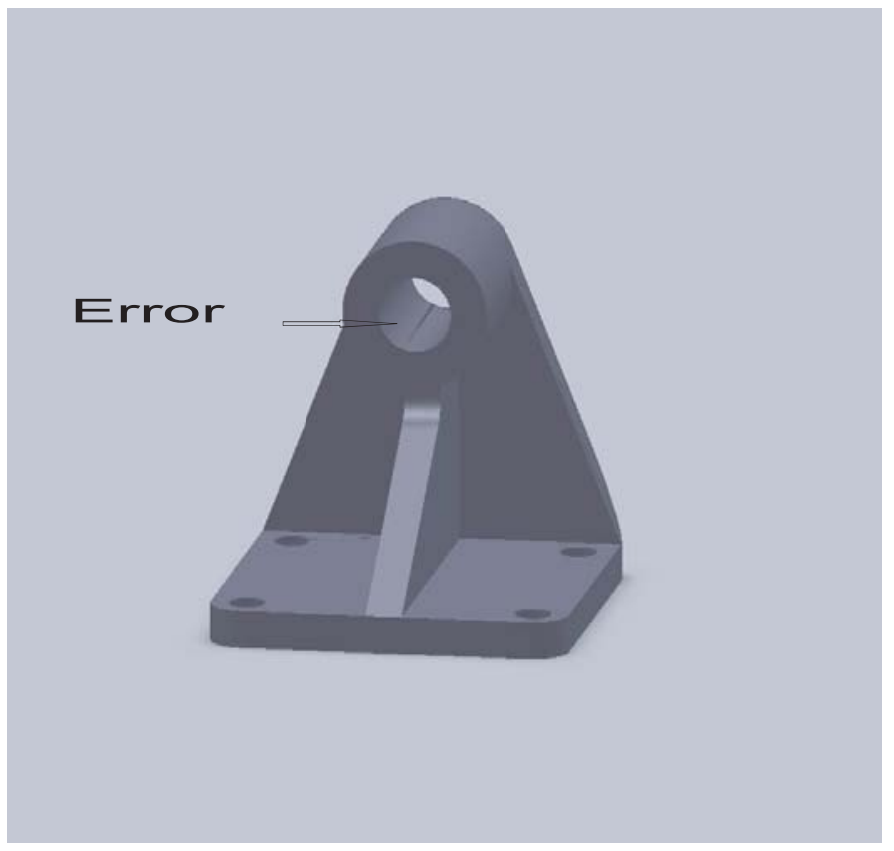

Figure 5.43: Damage Bracket SolidWork View.

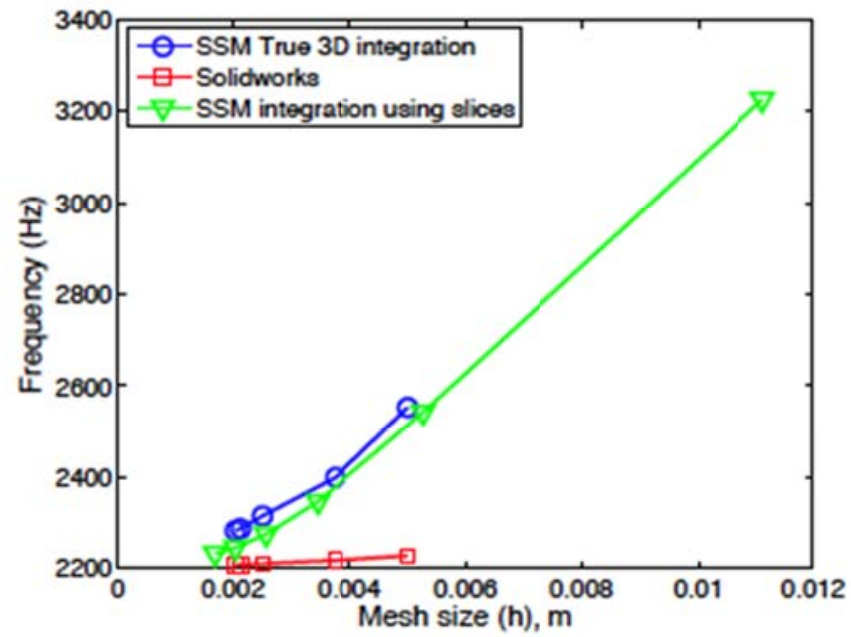

Figure 5.44: Damage Bracket Natural Vibration Mode 1. 


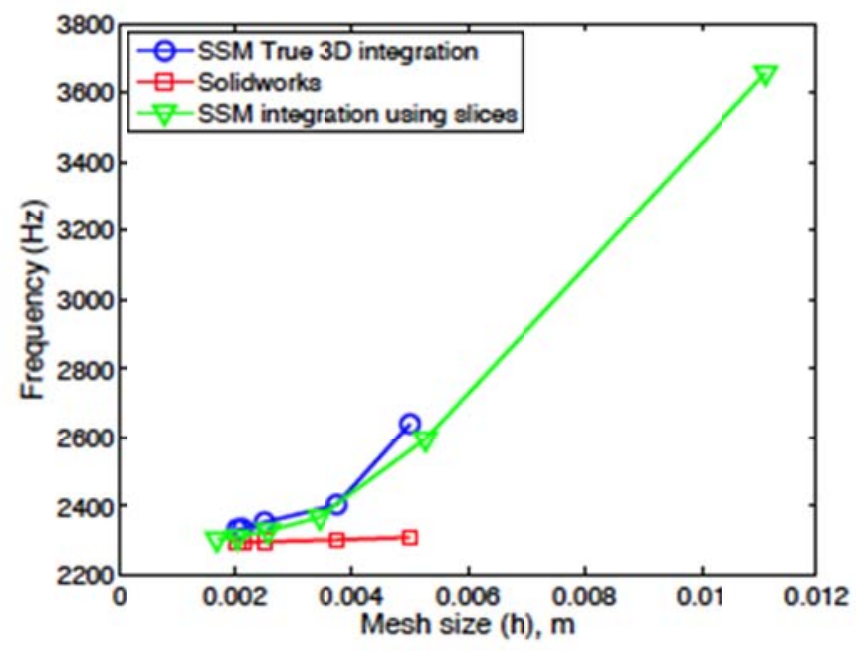

Figure 5.45: Damage Bracket Natural Vibration Mode 2.

convergence completely increases using mesh sizes toward the minimum value of $0.002 \mathrm{~m}$.

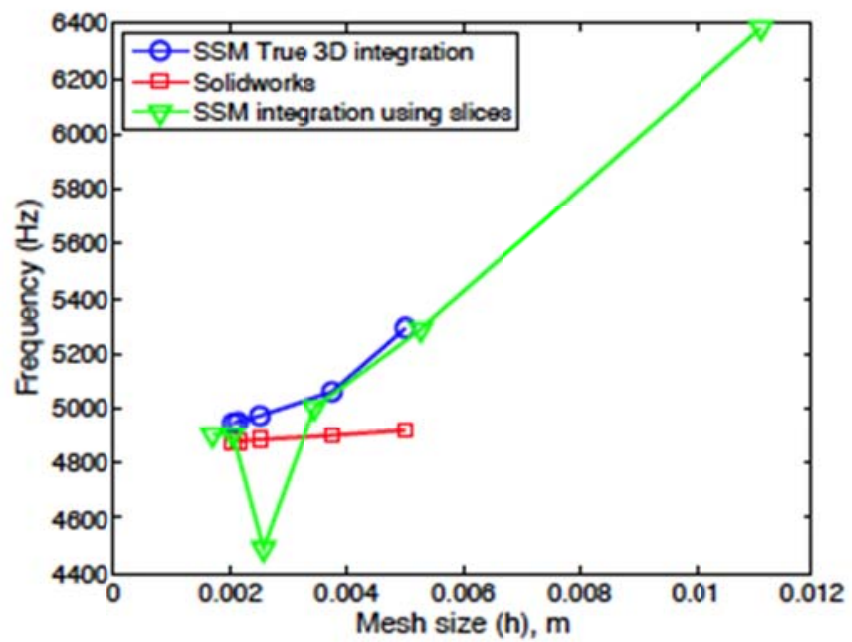

Figure 5.46: Damage Bracket Natural Vibration Mode 3.

For the third mode of natural vibration Figure5.46shows a convergence trend when we decrease the mesh size using the three integration methods. The slices method proposed shows a small trend toward deflexion probably produced by severe damages in the imperfect model. However, the slices integration method proposed reaches full convergence results and, similar to SOLIDWORKS and 3D, true integration at lower mesh sizes. 


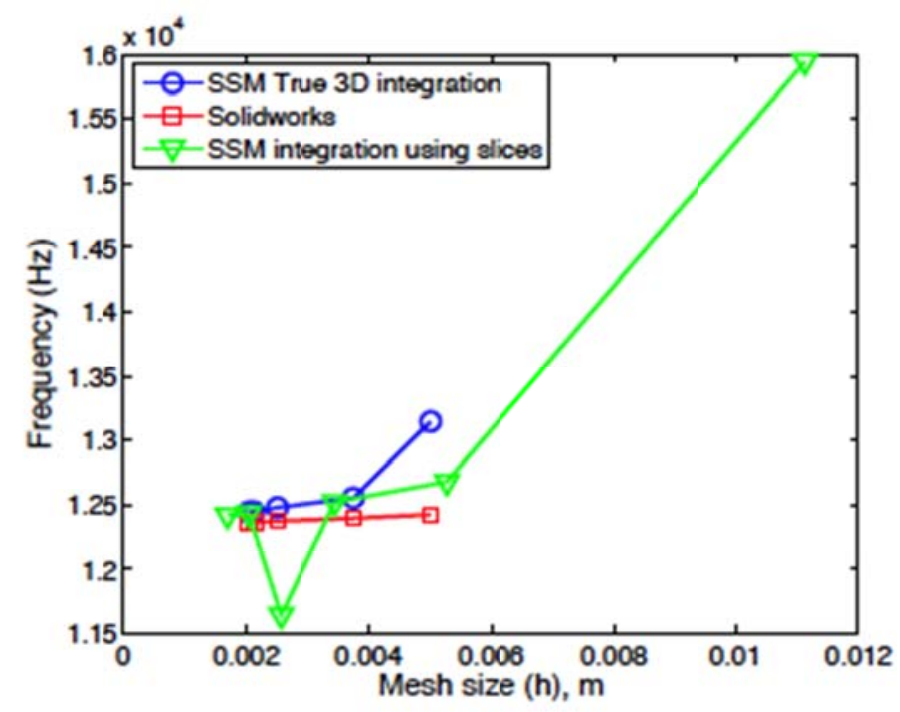

Figure 5.47: Damage Bracket Vibration Mode 10.

The natural vibration Figure5.47 for the mode 10 using the slices integration method in a bracket damaged model follow the same convergence trend as the convergence obtained using SOLIDWORKS and the true integration 3D methods, when reducing the mesh size. The convergence of the slices integration method proposed su ered a deflexion of its convergence trend when reaching lower mesh sizes, but again, as occurred at the third mode of vibration, the proposed integration algorithm recovered the path for a full convergence and similarity with SOLIDWORKS and 3D true integration. Causes of this local deflection convergence are created due to the increase of the natural frequencies values in a geometric damaged model.

The following 4 plots represents the convergence of the natural vibration modes experiments for an imprecise bracket model when compare with the solid work values

The Figure5.48 shows the convergence of the natural frequency Mode 1 to the Solid Work value when increasing grid refining

The Figure5.49 shows the convergence of the natural frequency Mode 2 to the Solid Work value when increasing grid refining

The Figure5.50 shows the convergence of the natural frequency Mode 3 to the Solid Work value when increasing grid refining 


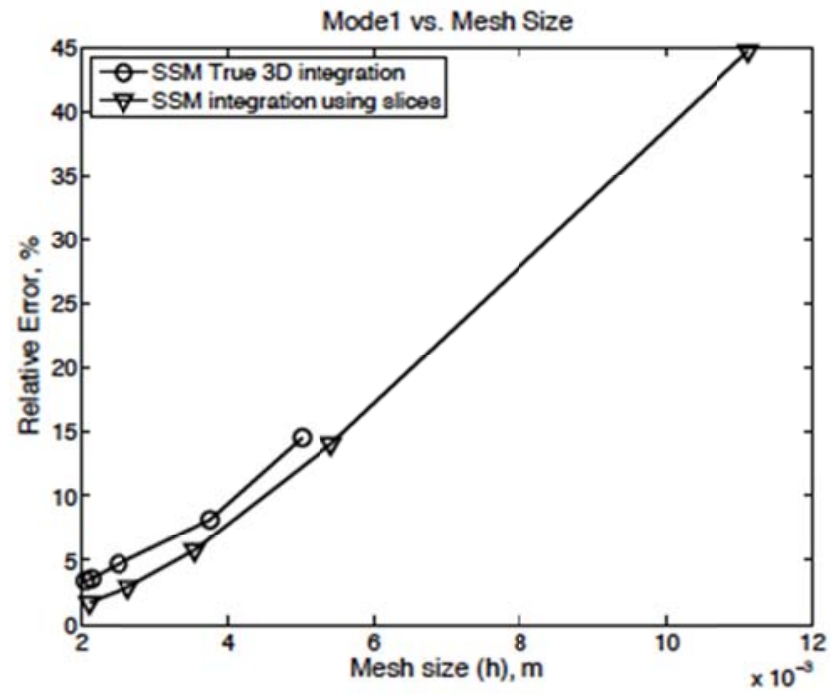

Figure 5.48: Damage Bracket Vibration Convergence Mode 1.

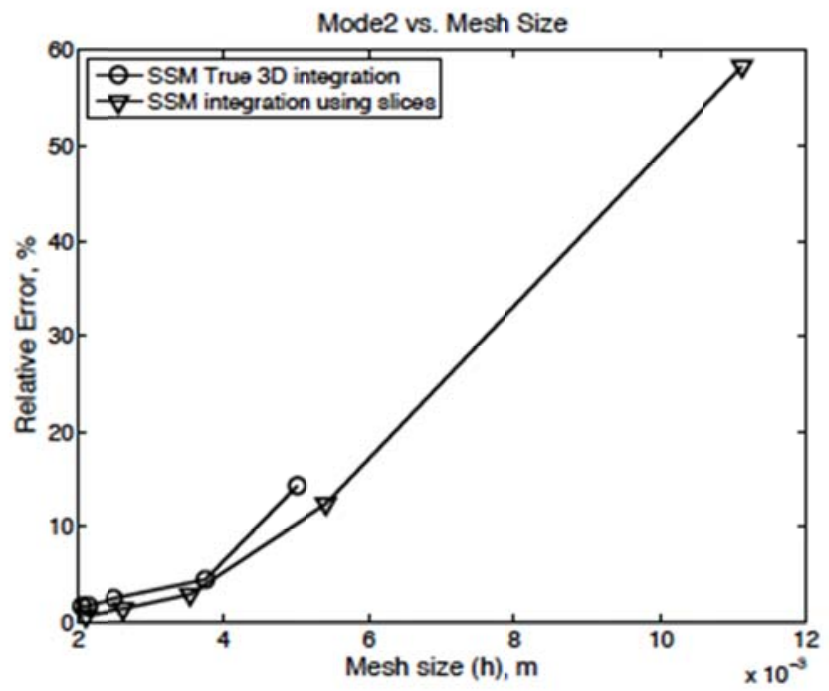

Figure 5.49: Damage Bracket Vibration Convergence Mode 2. 


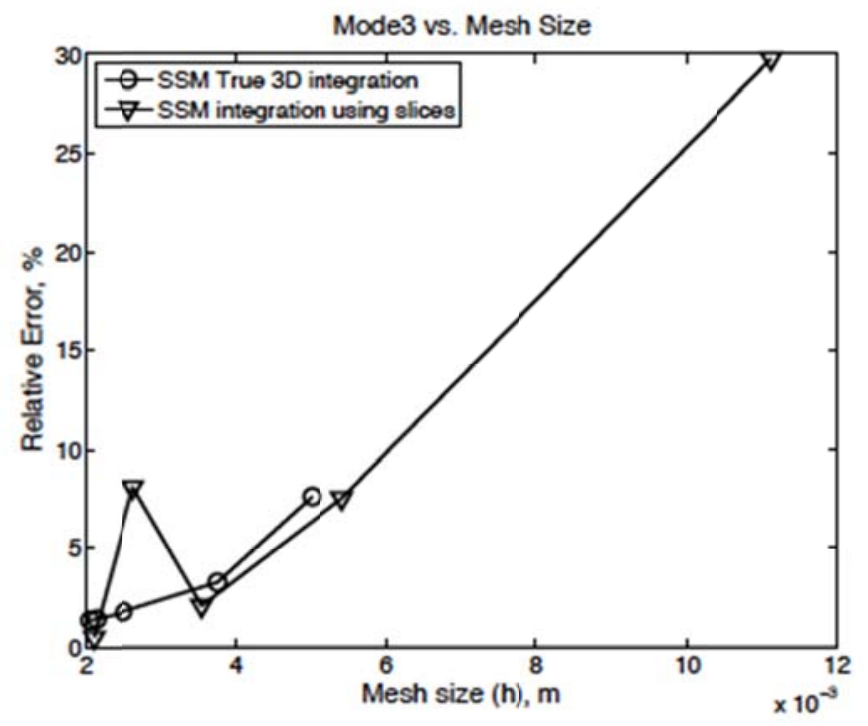

Figure 5.50: Damage Bracket Vibration Convergence Mode 3.

The Figure5.51 shows the convergence of the natural frequency Mode 10 to the Solid Work value when increasing grid refining

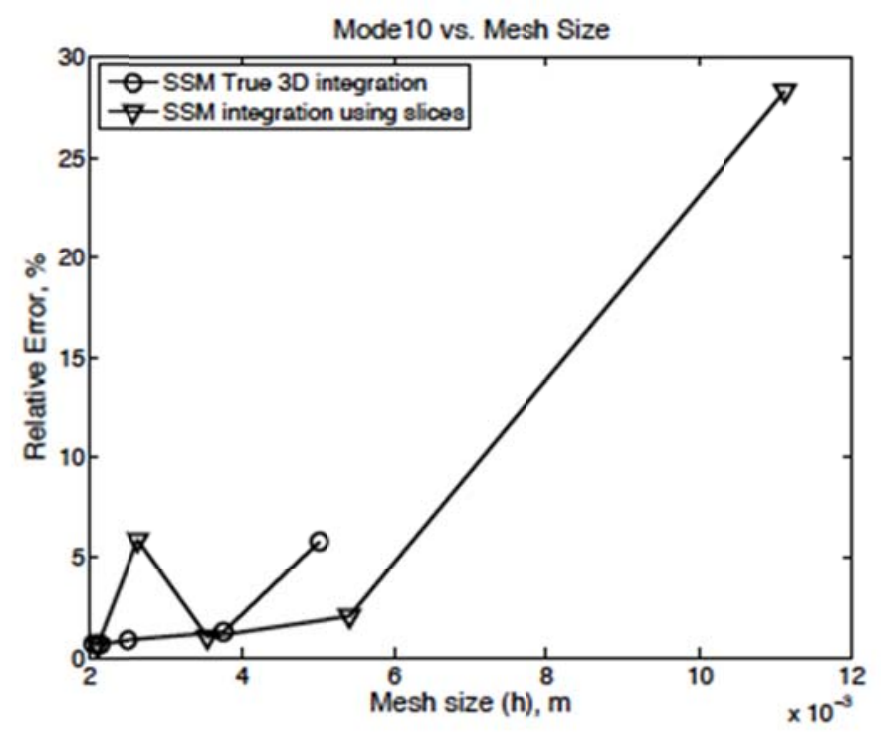

Figure 5.51: Damage Bracket Vibration Convergence Mode 10.

A precise model of a spider bracket will be show at Figure5.52 The model was designed in SOLIDWORKS and the Natural Vibration simulation tool was applied to obtain reference parameters to compare with the proposed integration by slices method. 


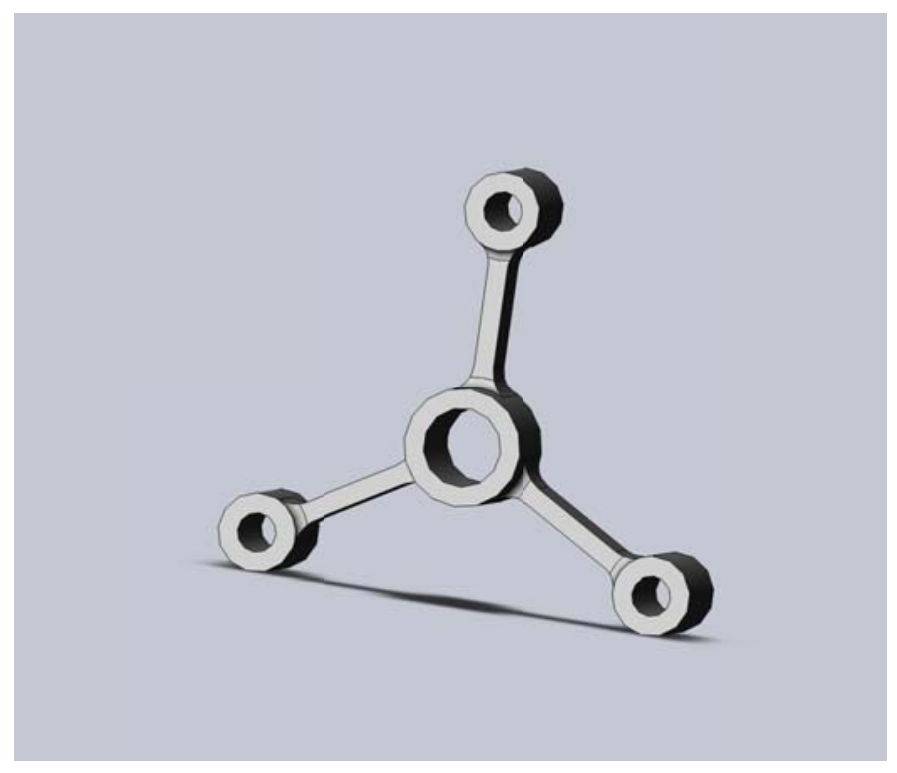

Figure 5.52: No Damage SpiderBracket Model.

The following plots represent natural vibration modes experiments for precise spider bracket models.

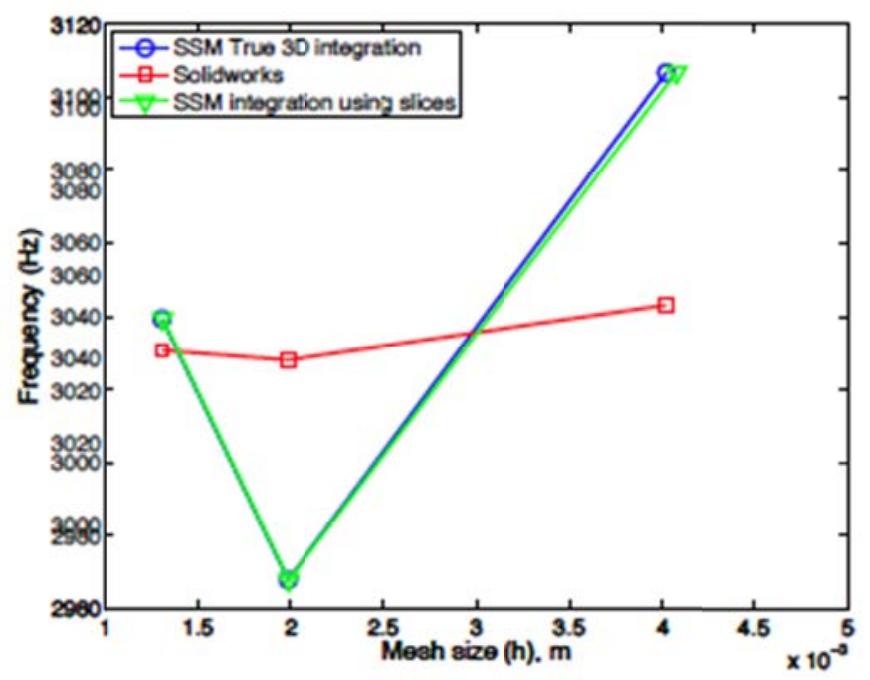

Figure 5.53: No Damage SpiderBracket Natural Vibration Mode 1.

The spider bracket model will be modeled using the SOLIDWORKS tool package as reference parameters, the true 3D integration method, and the proposed slicing integration method. For this first plot Figure5.53which represents the first mode of natural 
vibration for the precise model, we notice that there is a clear convergence using the proposed slicing method and the SOLIDWORKS reference when we reduce the mesh size. The 3D true integration method follows the same results obtained from the slices method.

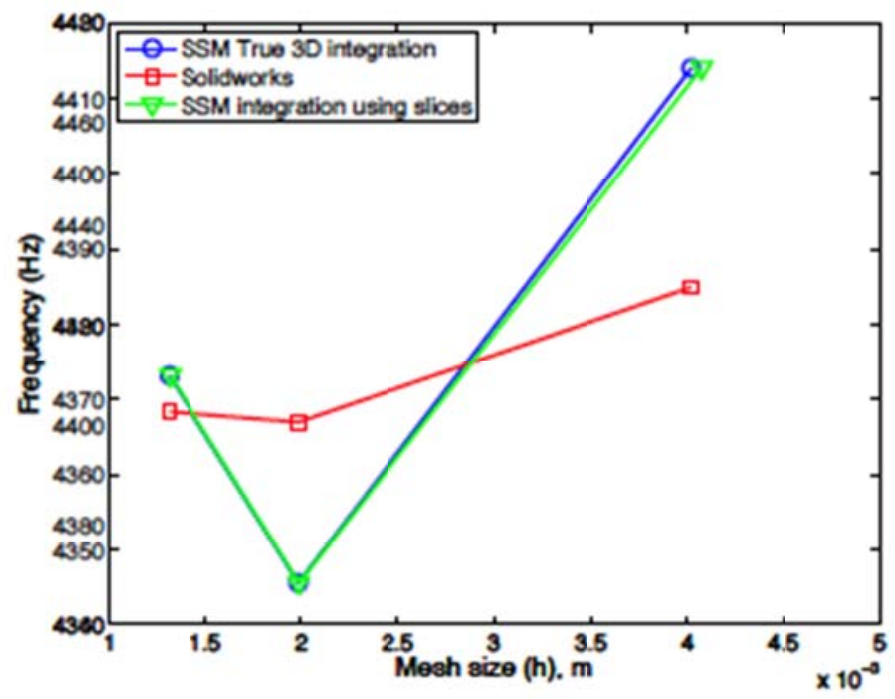

Figure 5.54: No Damage SpiderBracket Natural Vibration Mode 2.

For the second mode Figure5.54 of natural vibration, which has an increment of the value of the frequencies, we can notice that the convergence results match with the previous experiment. For the same mesh decreasing sizes we have obtained similar convergence results for a lower mesh size.

The results for mode 3 of the natural frequency Figure5.55using SOLIDWORKS corroborate the convergence findings between the slicing method proposed, the $3 \mathrm{D}$ true integration, and the SOLIDWORKS reference when decreasing the mesh size.

For the 10 mode of natural vibration Figure5.56 we can notice a full exact range of convergence between the slicing method and SOLIDWORKS. Both methods have parallel convergence using a relative lower mesh size.

The following 4 plots represents the convergence of the natural vibration modes experiments for a precise spider $_{b}$ racketmodelwhencomparewiththesolidworkvalues 


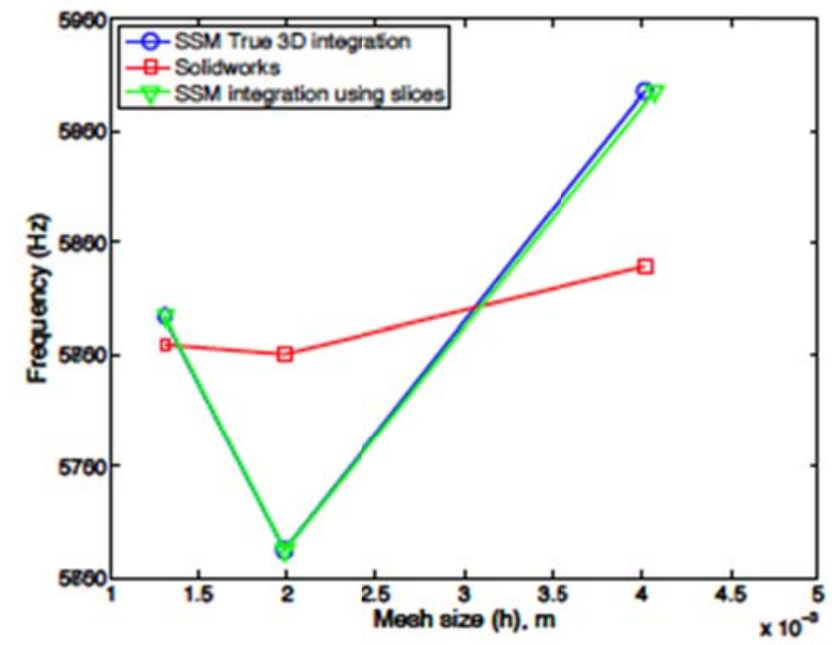

Figure 5.55: No Damage SpiderBracket Natural Vibration Mode 3.

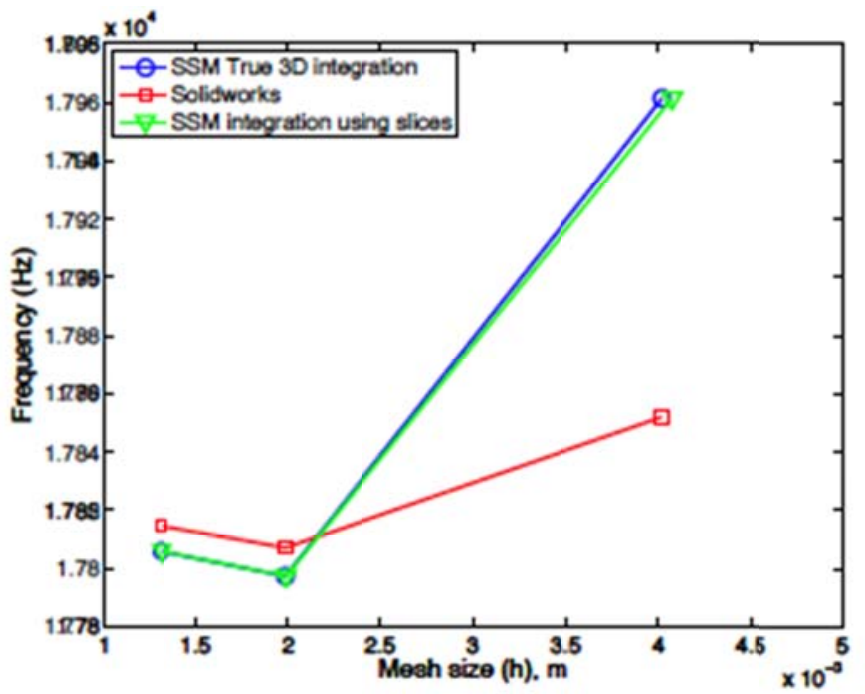

Figure 5.56: No Damage SpiderBracket Vibration Mode 10. 
The Figure5.57 shows the convergence of the natural frequency Mode 1 to the Solid Work value when increasing grid refining

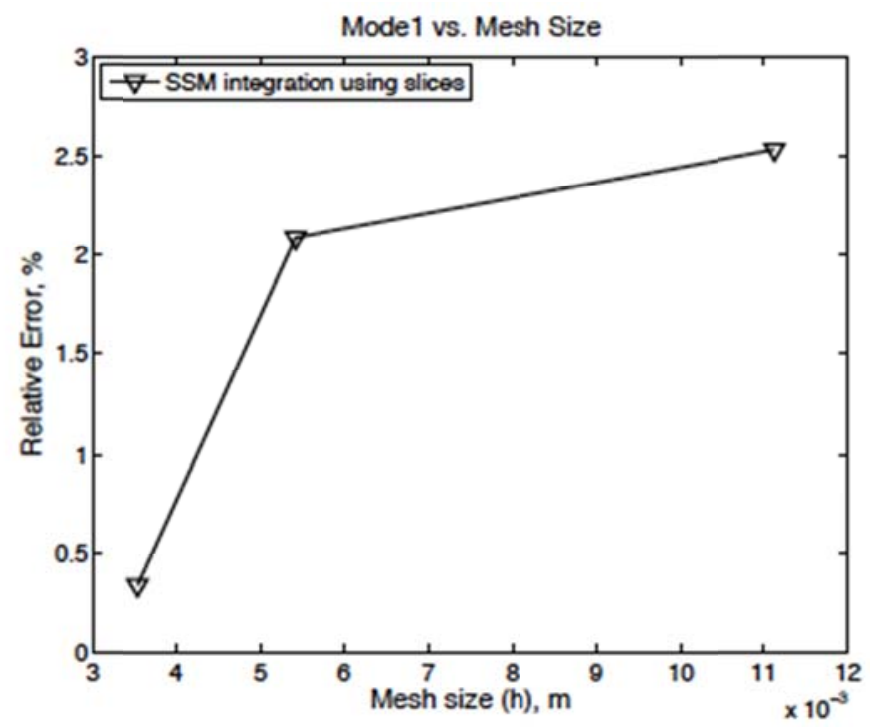

Figure 5.57: No Damage Spider Bracket Vibration Convergence Mode 1.

The Figure5.58 shows the convergence of the natural frequency Mode 2 to the Solid Work value when increasing grid refining

The Figure5.59 shows the convergence of the natural frequency Mode 3 to the Solid Work value when increasing grid refining

The Figure5.60 shows the convergence of the natural frequency Mode 10 to the Solid Work value when increasing grid refining

An imprecise model of a spider bracket will be shown in Figure5.61 The model was designed in SOLIDWORKS and the converter is an STL file. Once the stylographic model is opened, the triangulations surface shapes are allowed to manually damage the model. The following model has imprecise surfaces.

The following plots represent natural vibration modes experiments for imprecise spider bracket models.

The spider bracket imprecise model will be modeled again using the SOLIDWORKS tool package as reference parameters, a true integration 3D method, and the 


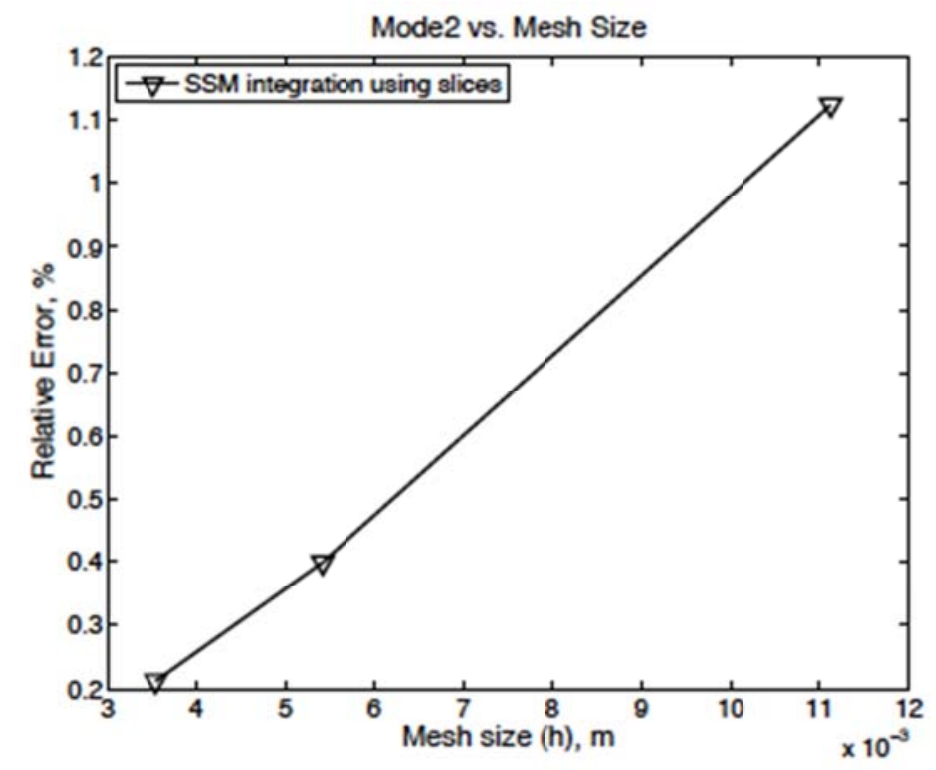

Figure 5.58: No Damage Spider Bracket Vibration Convergence Mode 2.

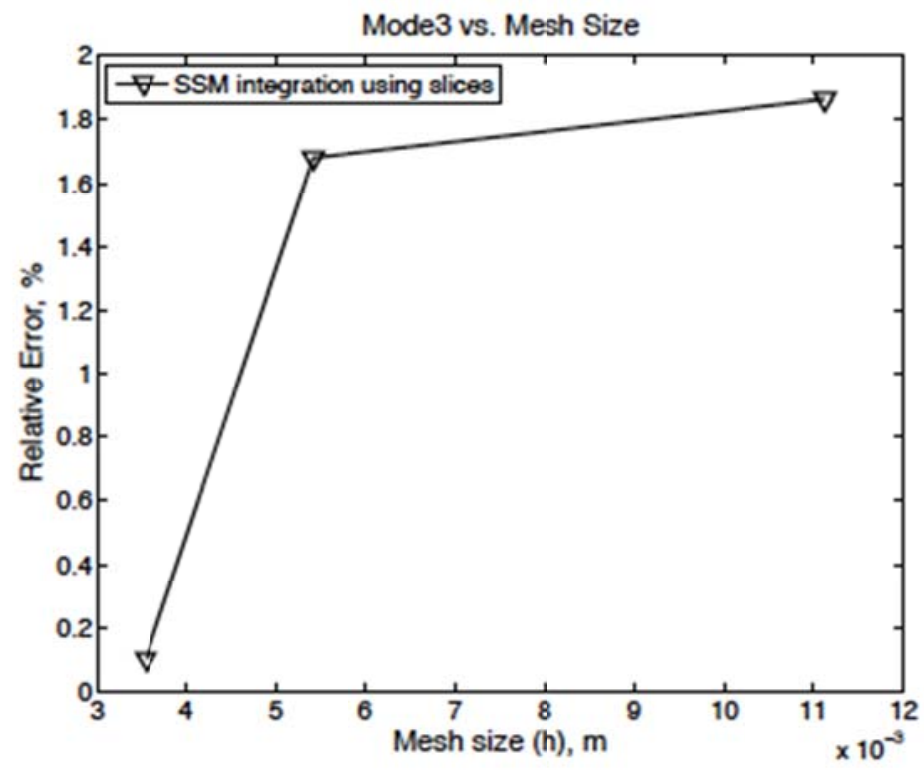

Figure 5.59: No Damage Spider Bracket Vibration Convergence Mode 3. 
Mode 10 vs. Mesh Size

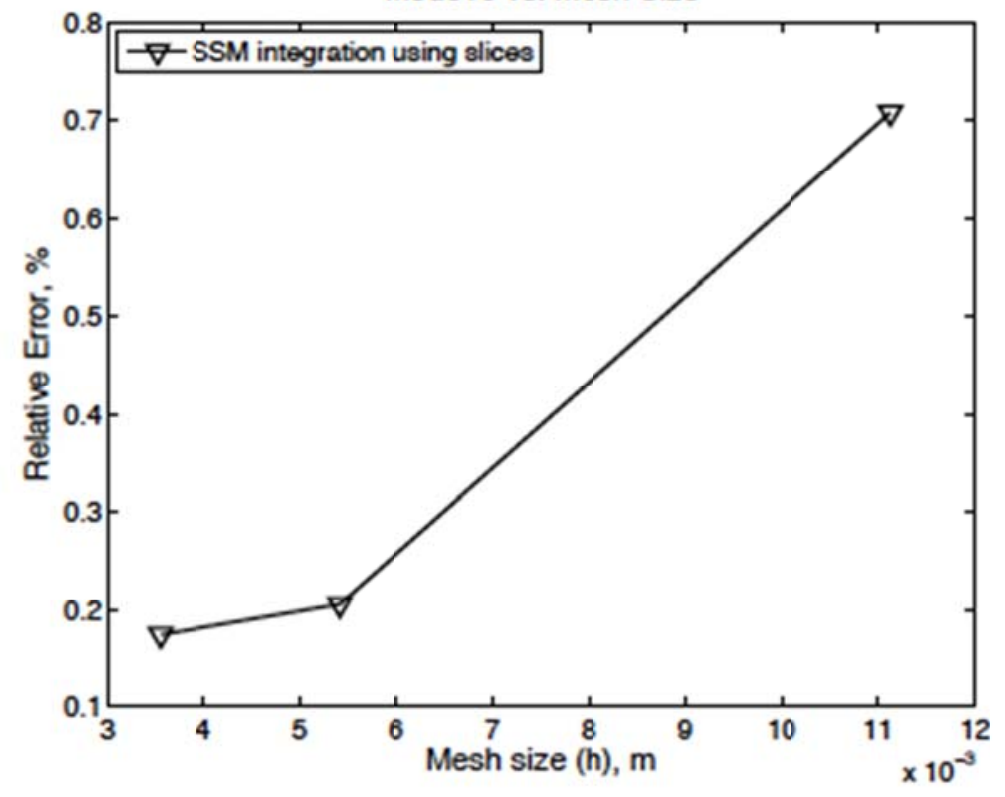

Figure 5.60: No Damage Spider Bracket Vibration Convergence Mode 10.

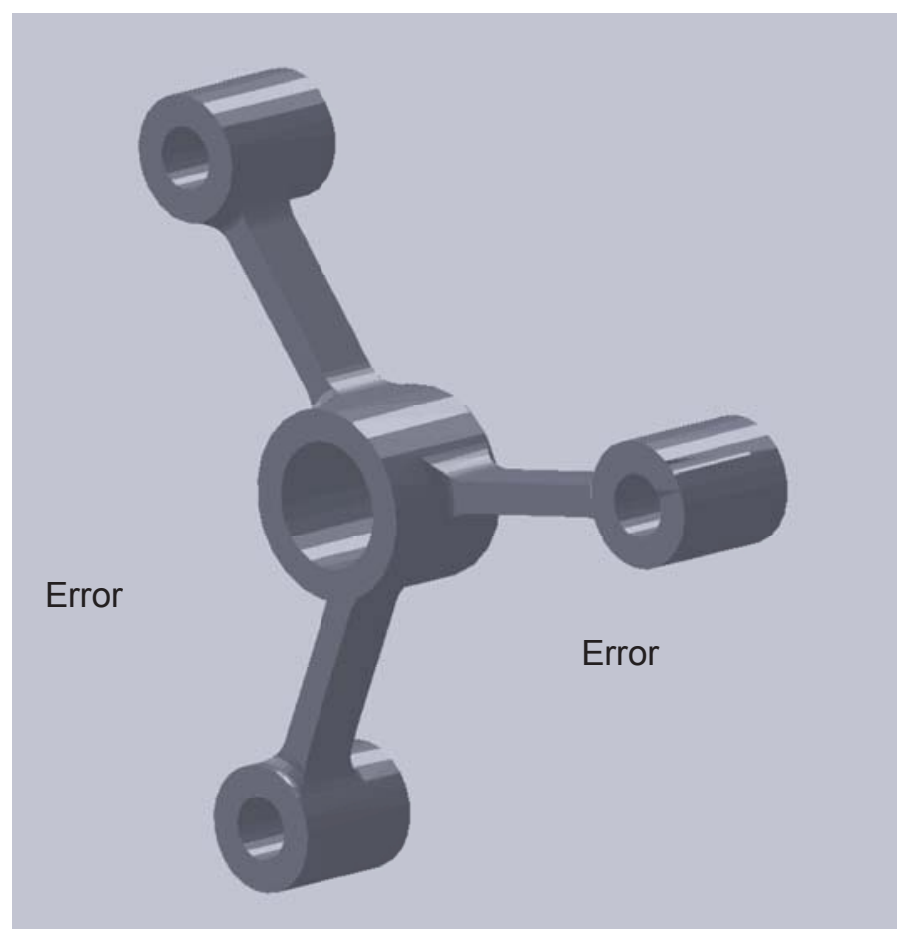

Figure 5.61: Damage SpiderBracket Model. 


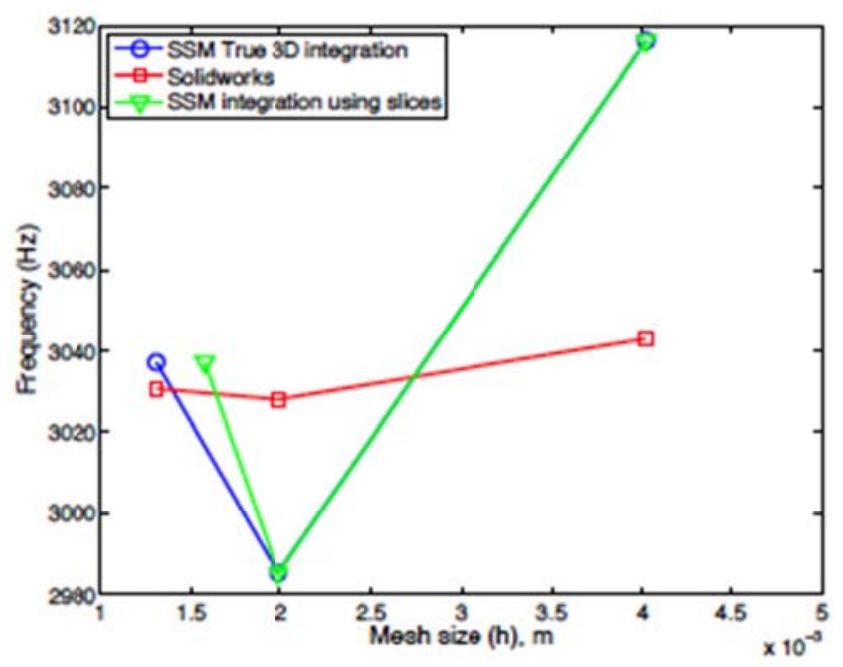

Figure 5.62: Damage SpiderBracket Natural Vibration Mode 1.

proposed slicing integration method. In this first plot, which represents the Mode 1 of vibration, Figure5.62 we can notice a full convergence of the proposed slices integration when reducing the mesh sizes. The true $3 \mathrm{D}$ integration method and the SOLIDWORKS tool also converge when we decrease the meshing size.

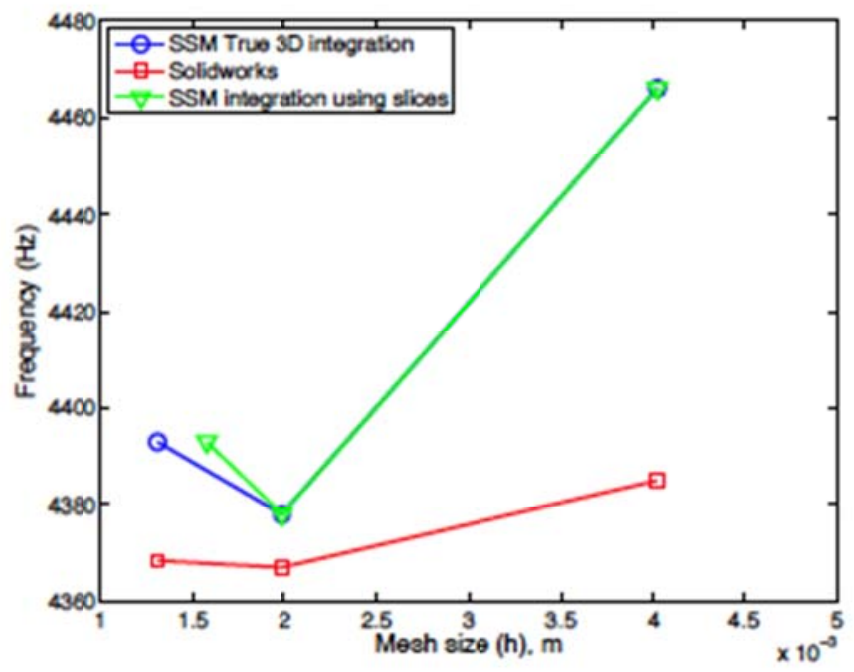

Figure 5.63: Damage SpiderBracket Natural Vibration Mode 2.

For the second mode Figure5.63 of natural vibration, which has an increment of the value of the frequencies, we can notice that the convergence trend matches with the previous mode of vibration, using the three integrative methods and when reducing mesh sizes. 


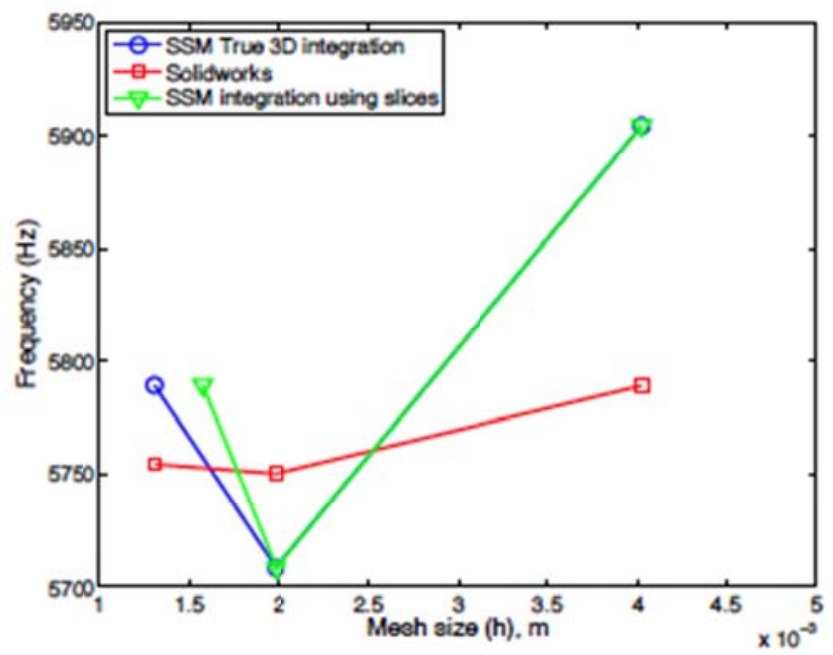

Figure 5.64: Damage SpiderBracket Natural Vibration Mode 3.

Results for mode 3 of the natural frequency Figure5.64using SOLIDWORKS corroborates the good convergence between the slicing method proposed and the SOLIDWORKS reference as lowering the mesh size. For mesh sizes between 0.0015 and 0.002 meters we obtain full convergence among the three methods.

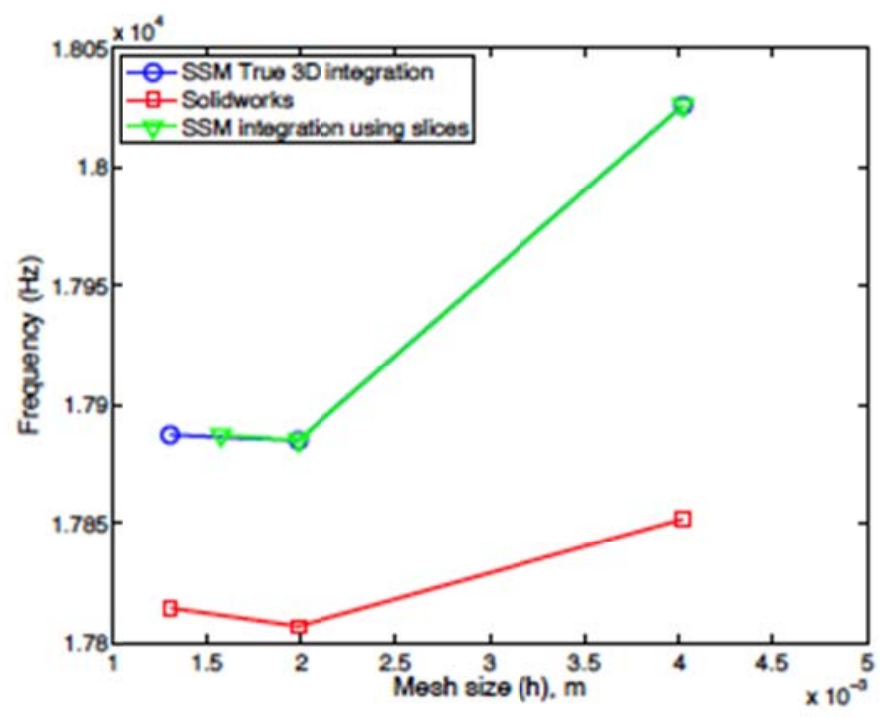

Figure 5.65: Damage SpiderBracket Natural Vibration Mode 10.

For the 10 mode of natural vibration Figure5.65 we can see that there is a parallel convergence between the proposed method and the SOLIDWORKS reference. The high natural vibration frequencies linked to the mode 10 and its di erence in value allows us 
to confirm a full convergence of both methods.

The following 4 plots represents the convergence of the natural vibration modes experiments for an imprecise bracket model when compare with the solid work values

The Figure5.66 shows the convergence of the natural frequency Mode 1 to the Solid Work value when increasing grid refining

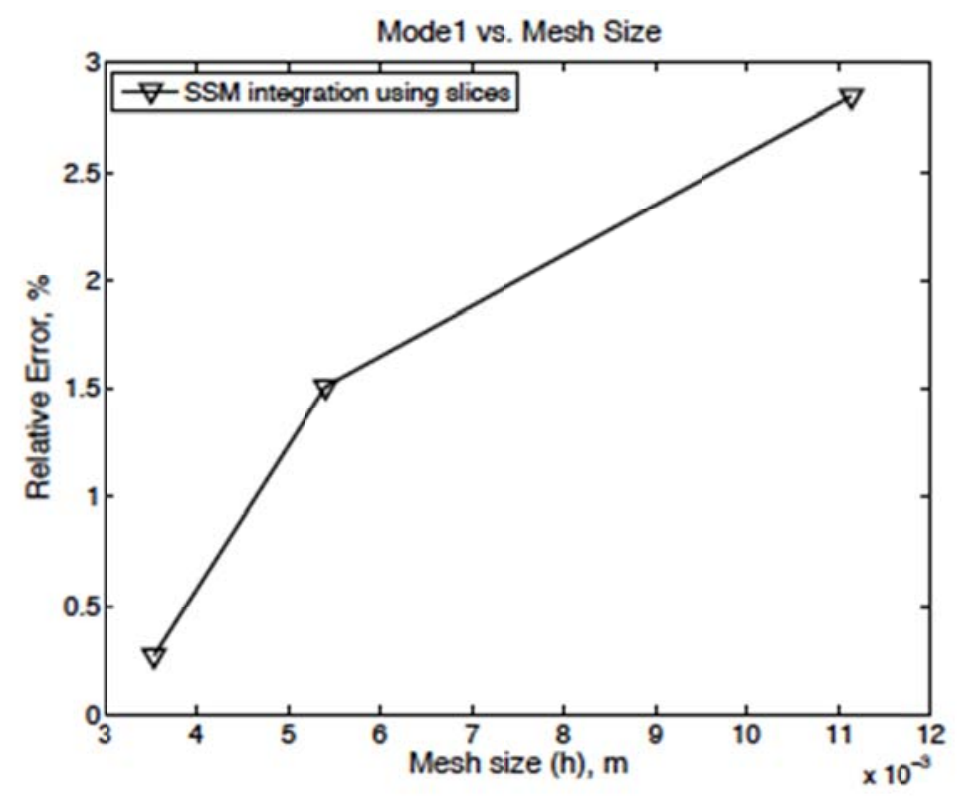

Figure 5.66: Damage Spider Bracket Vibration Convergence Mode 1.

The Figure5.67 shows the convergence of the natural frequency Mode 2 to the Solid Work value when increasing grid refining

The Figure5.68 shows the convergence of the natural frequency Mode 3 to the Solid Work value when increasing grid refining

The Figure5.69 shows the convergence of the natural frequency Mode 10 to the Solid Work value when increasing grid refining 


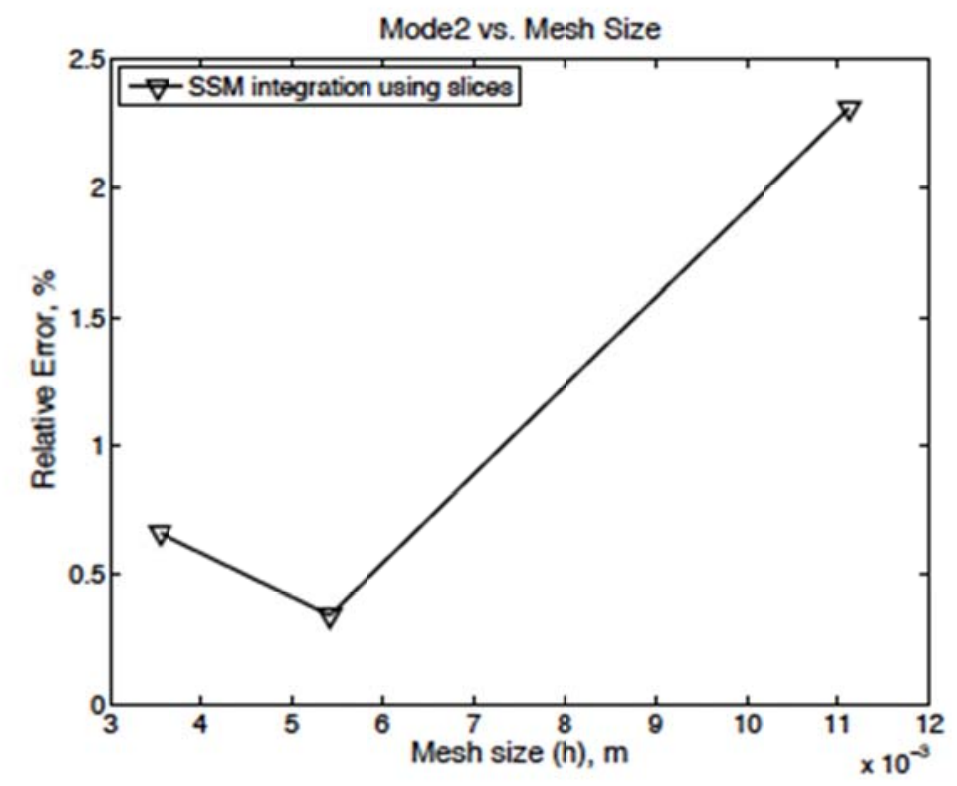

Figure 5.67: Damage Spider Bracket Vibration Convergence Mode 2.

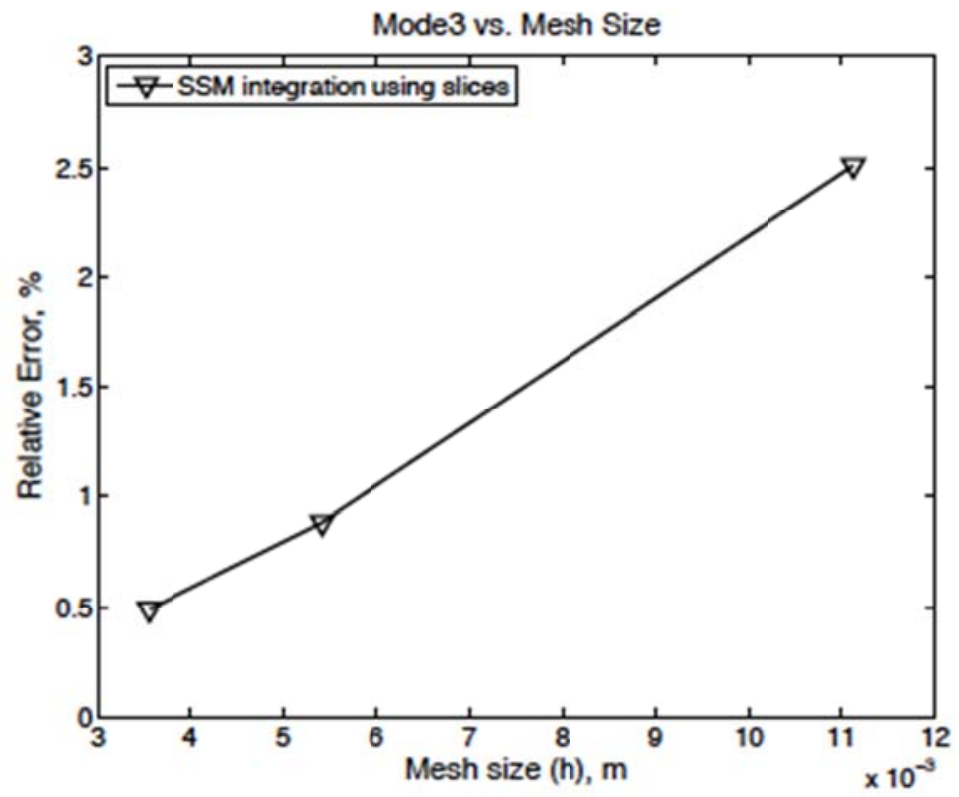

Figure 5.68: Damage Spider Bracket Vibration Convergence Mode 3. 


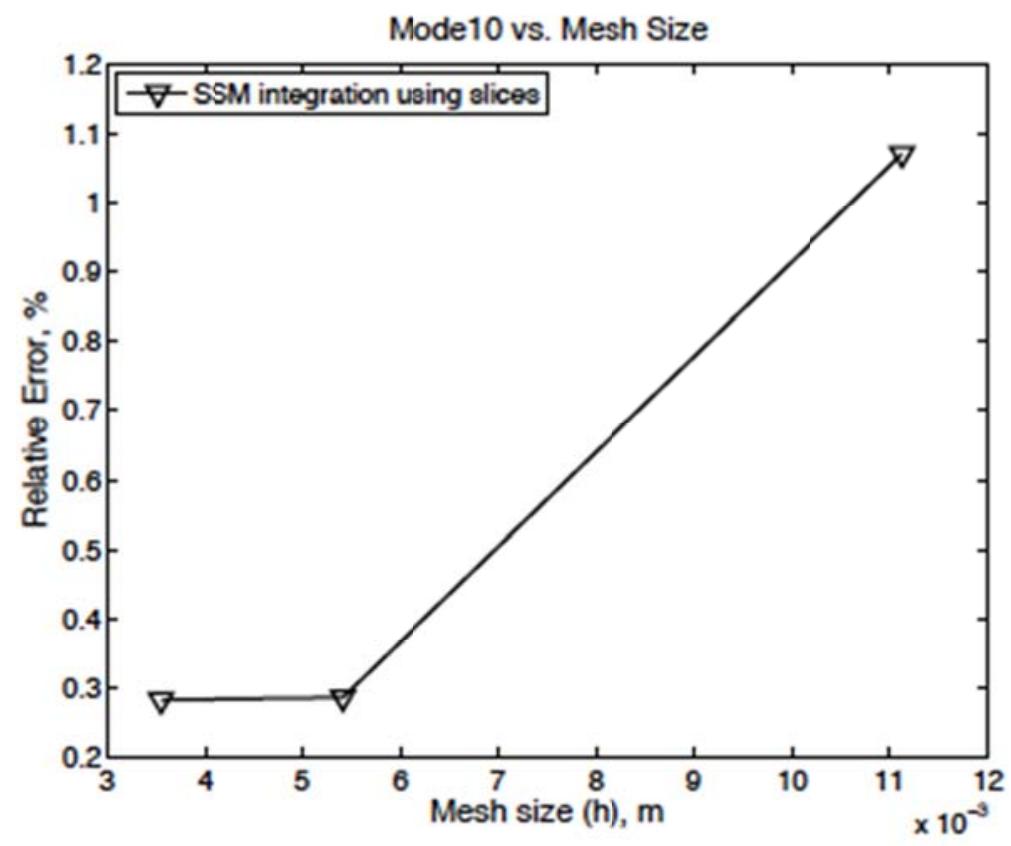

Figure 5.69: Damage Spider Bracket Vibration Convergence Mode 10. 


\section{CHAPTER 6}

\section{DISCUSSION AND CONCLUSIONS}

Computational mechanical analysis over geometric domains have been for many years a useful tool applied by engineers and researchers to obtain an approximate numerical engineering analysis for many physical problems where boundary conditions are applied in the geometry. This work is based on a meshfree method that uses a distance field approximation to enforce the boundaries' conditions. Geometric models are created by a CAD generator using drafting tools to create a solid. This geometry is conformed by a number of primitive elemental geometries, however a geometry generated in this way could contain imprecise or multiple lines intersections that force an eventual computational engineering analysis to stop the process and, in some cases, deal with geometrical repairs methods which are time expensive and usually change the entire geometric model configuration, including the boundaries conditions applied. The mechanical analysis method proposed in this dissertation uses an algorithm designed to compute geometric models with precise or imprecise geometries without any geometric repair necessary. Usually, these repairs are executed by an external software package that increases the process time and generates a geometric model distortion. The algorithm was created for 2D geometries and was extended to a 3D solid configuration. A solution structure is defined which contains the entire information about the boundaries' conditions and an implicit distance function that approximates the boundaries to enforce them. A boundary box is created conformed by 3D regular grids and will hold the model under analysis in order to allocate integration gauss points to support the governing equation of the physical problem. Hierarchical space decomposition is applied to every 2D grid parallel slice. Point Membership Classification (PMC) is computed for every slice iteratively. Cells with even or no intersections will be considered as regular cells. Cells with odd intersections will be considered as irregular cells and subject to 
a closing gap function without deforming or changing the proposed solid model. Integration gauss points are allocated into the cells and by slices once a full identification of internal, boundary, and external cells are computed. Gauss points will be allocated into the grids and each grid will be weighted to guarantee and fulfill the gauss allocation points for a 3D integration. The method proposed is completely automatic and no repairing geometry or healing method is needed. Verification and a number of experiments were processed to ensure the convergence of the proposed method and algorithms. The first set of experiments for volume calculation was made on perfect and imperfect models. The validation of the proposed algorithm will be centered first, to guarantee the closing gap method performance and ensure that no geometrical deformation was produced in the geometry, and second, using the gauss integration points allocation inside the regular cells, to assure a full volume convergence value when compared with the real one obtained from the SOLIDWORKS package. Di erent models were used with either perfect or damaged geometries. A simple geometry like a cylinder, or complex geometries like a Bracket or a Spur Gear, were processed using imperfect or damaged geometries. The closing gap and the integration slices methods proposed converge to the exact solution using large slices and gauss point integration. Plots shows that, independently, in the use of a simple or complex damaged model, the algorithm proposed has the capacity to converge to a solution very close, if not exact, to the real one, without any need for a repair software package. A second set of experiments was made in order to obtain a convergence validation of the algorithm package proposed. Natural vibration problems were applied in perfect and damaged geometries. The models used were the same bracket modeled for volume validation and a new proposed model called a spider bracket. For both models a study of the first 10 modes of vibration was done. A study was made for both models using the SOLIDWORKS packages, to compare values of the parameters. For this, studies were used only with the perfect models since the 
SOLIDWORKS program is incapable of accepting inputs with imperfections. Chapter 5 on validation and experimentation shows that the SOLIDWORKS solution package is incapable of handling models that are not fully closed,and terminates with the error message: Non solid model found. However, we compared the results using perfect models with the imperfect model results from our study. Using the bracket model for perfect and imperfect geometries we notice that the convergence of the frequencies are very alike when compared with the SOLIDWORKS package. Using the slicing method and comparing with a true 3D integration, we can see a full convergence of the solutions to the SOLIDWORKS value, inside the common range of the evaluation area. Frequencies are in the order of less than one percent with respect to the SOLIDWORKS value. The study for the spider bracket model, either for precise or imprecise models under natural vibration simulation, allows us to verify the convergence of the solution when compared to the SOLIDWORKS results. The algorithm for 3D models using precise or imprecise geometries agrees with the results obtained by the SOLIDWORKS package solver, noting one again that the SOLIDWORKS solver fails on models with geometrical imperfections. The geometrical imperfections manually created in the tested models were over the regular imperfection that can be created by the same engine package when the solid construction is created. The actual geometrical imperfections used were produced by the triangular shapes elimination from the stylographic geometrical representation of the solids. The order of magnitude of these imperfections is larger than a simple gap or an extended line and is usually created by well-known commercial design/solvers solid construction. Our algorithm proposed can also process any model coming from packages other than SOLIDWORKS, for example, the spur gear model imported by the GrabCAD solid model supplier. Our algorithm can be adapted or included in any other geometric generator engine package. The implementation in $\mathrm{C}++$ can be used as an engine solver in many areas of computational mechanics and can save time and money 
as a reliable package solver. 


\section{REFERENCES}

[1] 3D Systems, Inc. Stereolithography interface specification, 1988.

[2] Amenta, M.Bern, and M.Kamvysselis. A new voronoi based surfaced reconstructive algorithm. SIGGRAPH, 1998.

[3] S. Bischo and L. Kobbelt. Structure Preserving CAD Model Repair. In M. Alexa and J. Marks, editors, Computer Graphics Forum, volume 24, pages 527-536, 2005.

[4] Pavel Borodin, Marcin Novotni, and Reinhard Klein. Progressive gap closing for mesh repairing. In J. Vince and R. Earnshaw, editors, Advances in Modelling, Animation and Rendering, pages 201-213. Springer Verlag, July 2002.

[5] G. Butlin and C. Stops. CAD Data Repair. In Proceedings of the 5th International Meshing Roundtable, pages 7-12, 1996.

[6] C.S. Chong, A. Senthil Kumar, and H.P. Lee. Automatic mesh-healing technique for model repair and finite element model generation. Finite Elements in Analysis and Design, 43(15):1109 - 1119, 2007.

[7] Huong. Quynh. Dinh, Greg. Turk, and Greg. Slabaugh. Reconstructing surfaces by volumetric regularization. Smartech.gatech.edu, 2000.

[8] M. M. Ficco, F. Mandorli, and H. E. Otto. Error Classification and Recovery Within CAD Model Reconstruction. In SMA'99: Proceedings of the fifth ACM symposium on Solid modeling and applications, pages 316-317, Ann Arbor, MI, June 1999. ACM.

[9] J.D. Foley. Computer graphics: principles and practice. The Systems Programming Series. Addison-Wesley, 1996.

[10] S. Fortune and J.Hopcroft. Anote on rabin's nearest-neighbor algorithm. Informatuion Processing Letters, pages 20-23, 1979.

[11] M. Freytag, V. Shapiro, and I. Tsukanov. Field modeling with sampled distances. Computer Aided Design, 38(2):87-100, 2006.

[12] M. Freytag, V. Shapiro, and I. Tsukanov. Finite Element Analysis in Situ. Finite Elements in Analysis and Design, 47:957-972, 2011.

[13] R. Gasparini, T. Kosta, and I. Tsukanov. Engineering analysis in imprecise models. Finite Elements in Analysis and Design, 2012. Submitted for publication.

[14] Edelsbrunner H. and E.P. Mucke. Three dimensional alpha shapes. ACM Transaction on Graphics, 1994.

[15] T. Ju. Robust repair of polygonal models. ACM Transactions on Graphics, 23:888895, July 2004.

[16] T. Ju. Geometric Errors on Polygonal Models: A Survey. Journal of Computer Science and Technology, 24(1):19-29, 2009. 
[17] T. Ju, F. Losasso, S. Schaefer, and J. Warren. Dual contouring of hermite data. ACM Transactions on Graphics, 21:339-346, 2002.

[18] T. Kosta and I. Tsukanov. 3d natural vibration analysis with meshfree solution structure method. ASTM, page 7, 2014.

[19] Rvachev. V. L, T. Sheiko, V. Shapiro, and I. Tsukanov. Transfinite interpolation over implicity defined sets. Computer aided geometric design, 18:195-220, 2001.

[20] D. Li, A. Sobester, and A. J. Keane. A Knowledge-Based Geometry Repair System for Robust Parametric CAD Models. In 48th AIAA Aerospace Sciences Meeting Including the New Horizons Forum and Aerospace Exposition, Orlando, Florida, January 2010. AIAA. AIAA 2010-1508.

[21] W. E. Lorensen and H. E. Cline. Marching Cubes: A High Resolution 3D Surface Construction Algorithm. Computer Graphics, 21(3):163-169, July 1987.

[22] B. Luft, V. Shapiro, and I. Tsukanov. Geometrically adaptive numerical integration. In Proceedings of 2008 ACM Symposium on Solid and Physical Modeling, pages 147-157, Stony Brook, NY, June 2008.

[23] A.A. Mezentsev and T. Woehler. Aspects of automatic CAD model pre-processing and access in mesh generation. In N. Mastorakis, editor, System and control: theory and applications, World Multiconference on Circuits, Systems, Communications and Computers, pages 172-179. WSES Press, 2000.

[24] Andrey A. Mezentsev and Thomas Woehler. Methods And Algorithms Of Automated CAD Repair For Incremental Surface Meshing. In Proc. 8th Int. Meshing Roundtable, Sandia report SAND 99-2288, pages 299-309, 1999.

[25] M.Golin. Randomized data structure for the dynamic closest-pair problem. $A C M$ SIAM Symp, 26(4):301-310, 1998.

[26] F. S. Nooruddin and G. Turk. Simplification and repair of polygonal models using volumetric techniques. IEEE Transactions on Visualization and Computer Graphics, 9(2):191-205, April-June 2003.

[27] Paresh S. Patel, David L. Marcum, and Michael G. Remotigue. Stitching and filling: Creating conformal faceted geometry. In Proc. 14th Int. Meshing Roundtable, pages 239-256, 2005.

[28] P.Borodin, M.Novotni, and R.Klein. Progressive gap closing for mesh repairing. Springer, 2002.

[29] W. H. Press, S. A. Teukolsky, W. T. Vetterling, and B. P. Flannery. Numerical Recipes in C. Cambridge University Press, second edition, 1992.

[30] V. L. Rvachev. On analytical description of some geometric objects. Reports (Doklady) of Academy of Sciences, 153:765-768, 1963. USSR.

[31] V. L. Rvachev. Theory of R-functions and Some Applications. Naukova Dumka, 1982. In Russian. 
[32] V. L. Rvachev, T. I. Sheiko, V. Shapiro, and I. Tsukanov. On completeness of RFM solution structures. Computational Mechanics, 25:305-317, 2000.

[33] V. L. Rvachev and N. S. Sinekop. R-functions method in problems of the elasticity and plasticity theory. Nauk. dumka, Kiev, 1990. In Russian.

[34] V.L. Rvachev and L.V. Kurpa. R-functions in problems of the theory of plates. Nauk. dumka, Kiev, 1987. In Russian.

[35] M.I. Shamos and D.Hoey. Closest-point problems. Computer Methods in Applied Mechanics and Engineering, IEEE Symposium on Foundations of Computer Science:151-162, 1975.

[36] V. Shapiro and I. Tsukanov. Meshfree simulation of deforming domains. ComputerAided Design, 31(7):459-471, 1999.

[37] S.Levin, St.Levin, and J.Meisel. A dynamics analysis of the adoption of a new technology. The Review of Economics and Statistics, 1987.

[38] Brandon M. Smith, Timothy J. Tautges, and Paul P. H. Wilson. Sealing Faceted Surfaces to Achieve Watertight CAD Models. In Proc. 19th Int. Meshing Roundtable, pages 177-194, 2010.

[39] A. Sobester and A. J. Keane. Supervised Learning Approach to Parametric Computer-Aided Design Geometry Repair. AIAA JOURNAL, 44(2):282-289, February 2006.

[40] J. P. Steinbrenner, N. J. Wyman, and J. R. Chawner. Fast Surface Meshing on Imperfect CAD Models. In 9th International Meshing Rountable, page 9, 2000.

[41] Tao.Ju. Robust repair of polygonal models. SIGGRAPH 2004, 2004.

[42] I. Tsukanov and S. R. Posireddy. Hybrid method of engineering analysis: Combining meshfree method with distance fields and collocation technique. Journal of Computing and Information Science in Engineering, 11(2), 2011.

[43] I. Tsukanov and V. Shapiro. The architecture of SAGE - a meshfree system based on RFM. Engineering with Computers, 18(4):295-311, 2002.

[44] I. Tsukanov and V. Shapiro. Meshfree modeling and analysis of physical fields in heterogeneous media. Advances in Computational Mathematics, 23(1-2):95-124, November 2005.

[45] I. Tsukanov and V. Shapiro. Adaptive multiresolution refinement with distance fields. International Journal for Numerical Methods in Engineering, 72(11):13551386, 2007.

[46] I. Tsukanov, V. Shapiro, and S. Zhang. A meshfree method for incompressible fluid dynamics problems. International Journal for Numerical Methods in Engineering, 58(1):127-158, 2003. 
[47] I. G. Tsukanov. On the question of calculation of temperature fields in piecewise- homogeneous orthotropic media. Journal of Mathematical Sciences, 109(1):13381343, 2002.

[48] G. Turk and M. Levoy. Zippered polygon meshes from range images. In SIG-GRAPH'94, pages 311-318, Orlando, Florida, July 1994. ACM.

[49] Kantorovich L. V. and V.I. Krylov. Approximate methods of higher analysis.

1958. [50] Shapiro Vadim and Tsukanov Igor. Meshfree automation of engineering analysis. pages 127-136, 2002.

[51] G. Varadhan. Accurate minkowski sum approximation of polyhedral methods. El-sevier, 68:343-355, 2006.

[52] T. Varadya, R. R. Martina, and J. Cox. Reverse engineering of geometric models an introduction. Computer-Aided Design, 29(4):255-268, April 1997.

[53] Z.J. Wang and K. Srinivasan. An adaptive Cartesian grid generation method for

Dirty geometry. International Journal for Numerical Methods in Fluids, 39:703717, 2002.

[54] W.Schroeder, J. Zarge, and W. Lorensen. Decimation of triangles meshes. Computer Graphics, 1992.

[55] Guoliang. Xu, Qing. Pan, and Chandrajit. Bajaj. Discrete surface modeling using geometric flows. Chinese Academy of Sciences, 2003.

[56] J. Yang and S. Han. Repairing CAD model errors based on the design history. Computer-Aided Design, 38(6):627-640, June 2006.

[57] O. Yutaka and Hans. Seidel. A Multi-scale Approach to 3D Scattered Data Inter- polation with Compactly Supported Basis Functions. IEEE, 2003.

[58] Borut Zalik. An e cient sweep-line delaunay triangulation algorithm. Elsevier, 2004. 
VITA

\section{RICCARDO GASPARINI}

Ph.D. Mechanical Engineering

Florida International University

2013-2015

* Professional Engineer State of Florida Lic \#60779.

Professional Practice

2010-2013

Research Assistant, Teaching Assistant, FlU School of Engineering

* Engineering Analysis in Imprecise Geometric Models. Meshfree Analysis

*Computational Mechanics

2010

Robotic Engineer Certification

December 2009

Master of Science Mechanical Engineering

University of Simon Bolivar

1985

Certification Mechanical Engineering,

1984

Mechanical Engineering, 5-ycar degree, University of Bologna, Italy

Old Employments:

*Development of On-Grid Solution Green Power.

Development of a wind and solar power system as an hybrid source for domestic use *Silence and Static Portable power generator manufacturing for indoor and outdoor use.

Commercial and Residential

*Hybrid Super capacitors -Batteries storage energy

Project Engineer, Testwell Laboratories, New York and Ft. Lauderdale

* Responsible for civil and Mechanical construction operations in South Florida . Material testing company. Managed concrete/steel test certification. Ground penetration radar. 
*ASTM protocols. UL Fire stopping testing and application

Chief Engineer if Operations, South Florida Packaging, Miami, Florida

* Production of paper and plastic bags; Design of automatic folder machines; installation and Run injection molding machines.

Flexographic: press machines

Design Engineer, Unitrend Power Technologies, Miami, Florida

*Design and Developed an Electronic Ballast for High Intense

Discharge for Outdoor application

Release a Patent: \#5,798,615 (USA and Europe).And UL

protocols for indoor applications

- Other 1lreas Covered (Professional Activities in Europe):

- Design and developed Electric Panel. $\mid u\rfloor$ ill PLC systems and CNC controllers

- Developed DC motors for auto motor industries

- Inverters controllersfor Asynchronous motors for Auto motor industry

- Develop Aluminum Heat exchanger (oil-Air) for Auto motor industry

- Develop Electrostatic Generators for Paint Chamber equipments

- Design Ultrasounds cells for moisture generation for food conservation Industries

- Vacuum Metallization.for Ca pacitors manfacturer industries

- Industrial Controllers for energy saving

- Electromagnetic coil design for Power transmissions

Universal Electronic Ballast Patent \#5,798,615 (USA and Europe)

\section{PUBLICATIONS}

- Imprecise Model Analysis in Com putational Mechanics

- 'Wireless Elcctromagru.ti...; Charger for Domestic Robot 